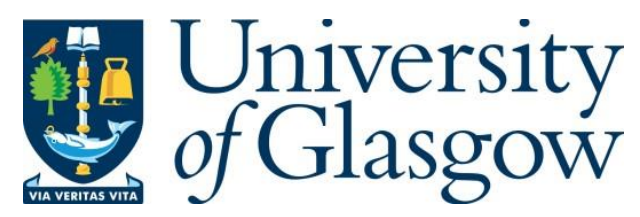

Ortigosa, R., Gil, A. J. and Lee, C. H. (2016) A computational framework for large strain nearly and truly incompressible electromechanics based on convex multi-variable strain energies. Computer Methods in Applied Mechanics and Engineering, 310, pp. 297-334.

There may be differences between this version and the published version. You are advised to consult the publisher's version if you wish to cite from it.

$\underline{\text { http://eprints.gla.ac.uk/168847/ }}$

Deposited on: 14 September 2018

Enlighten - Research publications by members of the University of Glasgow http://eprints.gla.ac.uk 


\section{Accepted Manuscript}

A computational framework for large strain nearly and truly incompressible electromechanics based on convex multi-variable strain energies

Rogelio Ortigosa, Antonio J. Gil, Chun Hean Lee

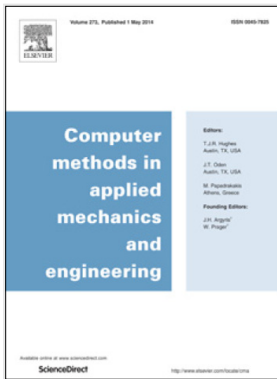

PII:

S0045-7825(16)30228-6

DOI: $\quad$ http://dx.doi.org/10.1016/j.cma.2016.06.025

Reference: CMA 11026

To appear in: Comput. Methods Appl. Mech. Engrg.

Received date: 20 April 2016

Revised date: 23 June 2016

Accepted date: 24 June 2016

Please cite this article as: R. Ortigosa, A.J. Gil, C.H. Lee, A computational framework for large strain nearly and truly incompressible electromechanics based on convex multi-variable strain energies, Comput. Methods Appl. Mech. Engrg. (2016), http://dx.doi.org/10.1016/j.cma.2016.06.025

This is a PDF file of an unedited manuscript that has been accepted for publication. As a service to our customers we are providing this early version of the manuscript. The manuscript will undergo copyediting, typesetting, and review of the resulting proof before it is published in its final form. Please note that during the production process errors may be discovered which could affect the content, and all legal disclaimers that apply to the journal pertain. 


\title{
A computational framework for large strain nearly and truly incompressible electromechanics based on convex multi-variable strain energies.
}

\author{
Rogelio Ortigosa ${ }^{1}$, Antonio J. Gil ${ }^{2}$, Chun Hean Lee \\ Zienkiewicz Centre for Computational Engineering, College of Engineering \\ Swansea University, Bay Campus, SA1 8EN, United Kingdom
}

\begin{abstract}
The series of papers published by Gil and Ortigosa [1-3] introduced a new convex multi-variable variational and computational framework for the numerical simulation of Electro Active Polymers (EAPs) in scenarios characterised by extreme deformations and/or extreme electric fields. Building upon this body of work, five key novelties are incorporated in this paper. First, a generalisation of the concept of multi-variable convexity to energy functionals additively decomposed into isochoric and volumetric components. This decomposition is typical of nearly and truly incompressible materials, group which represents the majority of the most relevant EAPs. Second, convexification or regularisation strategies are applied to a priori non-convex multi-variable isochoric functionals to yield physically meaningful convex multi-variable functionals. Third, based on the mixed variational principles introduced in Reference [1] in the context of compressible electro-elasticity, a novel extended $\mathrm{Hu}$-Washizu mixed variational principle for nearly and truly incompressible scenarios is presented. From the computational standpoint, a static condensation procedure is applied in order to condense out the element-wise extra fields, the resulting formulation having a comparable cost to the more standard three-field displacement-potential-pressure mixed formulation. Fourth, the computational framework for the three-field mixed variational principle in nearly and truly incompressible scenarios is also presented. In this case, the novelty resides in the consideration of convex multi-
\end{abstract}

\footnotetext{
${ }^{1}$ Corresponding author: r.ortigosa@swansea.ac.uk

${ }^{2}$ Corresponding author: a.j.gil@swansea.ac.uk
} 
variable energy functionals. Ultimately, this leads to the definition of new tangent operators for the Helmholtz's energy functional in the specific context of incompressible electro-elasticity. Fifth, a Petrov-Galerkin stabilisation technique is applied on the three-field formulation for the circumvention of the Ladyženskaja-Babuška-Brezzi (LBB) condition, enabling the use of linear tetrahedral finite elements for the interpolation of the unknowns of the problem. Finally, a series of challenging numerical examples is presented in order to provide an exhaustive comparison of the different variational formulations presented in this paper.

Keywords: Dielectric elastomers, Nonlinear electro-elasticity, material stability, Mixed variational principles, polyconvexity, incompressibility

\section{Introduction}

Dielectric elastomers (DEs) belong to a general class of Electro Active Polymers (EAPs) with remarkable actuation properties [4-7]. Recently, Li et al. [8] have reported an outstanding voltage induced area expansion of $1980 \%$ on a Dielectric Elastomer membrane film. In this specific case, the electromechanical instability is harnessed as a means for obtaining these electrically induced massive deformations with potential applications in soft robots, adaptive optics, balloon catheters and Braille displays [8], among others. Moreover, these materials have been successfully applied as generators to harvest energy from renewable sources, such as human movements and ocean waves [9].

Several authors [10-24] have contributed to the development of a well established variational framework for the numerical simulation of electro active materials. Crucial to this variational framework is the definition of well posed constitutive equations, as these materials are prone to develop a variety of electromechanical instabilities [25]. Among these, it is vital to identify those material instabilities associated to the onset of macroscopic instabilities [26-28]. The latter are related to the loss of ellipticity [29, 30] and the loss of positive definiteness of the generalised acoustic tensor [26, 27, 31] and, ultimately, lead to the ill-posedness of the governing equations. From the numerical standpoint, these detrimental features yield [25-27] a pathological mesh dependence behaviour similar to that observed in the modelling of strain localisation [32]. 
In nonlinear elasticity, ellipticity of the constitutive model is satisfied ab initio by polyconvex [29, 30, 33-39, 39-51] energy functionals. Gil and Ortigosa [1] extended the concept of polyconvexity to finite strain electromechanics, where the more appropriate term multi-variable convexity was adopted. The authors defined a new electro-kinematic variable set including the deformation gradient tensor $\boldsymbol{F}$, its adjoint or co-factor $\boldsymbol{H}$, its determinant $J$, the Lagrangian electric displacement field $\boldsymbol{D}_{0}$ and an additional spatial or Eulerian electromechanical variable denoted as $\boldsymbol{d}$. Convexity of the internal energy functional with respect to the elements of this extended set permits an extension of the concept of ellipticity [30] not only to the entire range of deformations but to any applied electric field.

In the present manuscript, the concept of multi-variable convexity is generalised to energy functionals which are additively decomposed into isochoric and volumetric components. Notice that many authors $[46,52,53]$ advocate for this approach in order to model the behaviour of nearly and truly incompressible materials, as is the case of the popular acrylic elastomer VHB 4910. Based on the work in Reference [1], some strategies to create appropriate convex multi-variable isochoric invariants by incorporating minor modifications to a priori non-convex multi-variable isochoric invariants are presented in this work.

From the numerical standpoint, in the context of incompressible elasticity, some authors resort to interpolation spaces for both geometry and pressure fields which a priori do not satisfy the Ladyženskaja-Babuška-Brezzi (LBB) [54-56] condition but then recover the ellipticity of the problem via appropriate stabilisation techniques [41, 47, 53, 57-63]. In this regard, the Stream-Upwind-Petrov-Galerkin (SUPG) method can be utilised as a robust technique for the circumvention of the LBB condition [64]. On the contrary, other authors resort to Finite Elements discretisations which satisfy the LBB condition condition, as for instance, the P2P1 and Q2Q1 Taylor-Hood elements [55].

In the context of incompressible electro-elasticity, some authors [65] resort to the well established $B$-bar [66] element for the discretisation of the standard three-field displacement-potential-pressure variational principle. In this element, a bilinear/trilinear (for 2D/3D applications) interpolation of the geometry and electric potential and a constant element by element interpolation of the pressure field is considered. Unfortunately, this choice of interpolation spaces does not satisfy the LBB condition and can potentially lead to spurious pressure modes [67]. The use of pressure smoothing at a 
postprocessing stage [68] can help alleviate this shortcoming. In conformity with this non-LBB compliant line of research, we present the SUPG stabilisation of the three-field variational principle which allows for the use of linear tetrahedral finite elements for all the unknowns of the problem.

Additionally, following Gil and Ortigosa [1, 2], we propose an extended Hu-Washizu [44, 52, 54, 54, 69-71, 71-80] mixed variational principle for nearly and truly incompressible scenarios. Considering now interpolation spaces which satisfy the LBB condition, a comparison of this enhanced methodology is carried out in this paper against the three-field formulation, where both LBB compliant and non-compliant interpolation spaces are considered.

The paper is organised as follows. Section 2 revises the fundamental equations of electromechanics with the help of the tensor cross product notation introduced by de Boer [81] and later applied by Bonet et al. [46, 82] in the context of nonlinear continuum mechanics. Very succinctly, this Section revisits the concept of multi-variable convexity introduced in Reference [1] and its particularisation to energy functionals additively decomposed into isochoric and volumetric components. Moreover, this Section presents the tangent operators of both the internal energy and the Helmholtz's energy functional based on the aforementioned decomposition. Section 3 presents a series of mixed variational principles suitable for nearly and truly incompressible electromechanics. The Finite Element computational implementation of these formulations is presented in this Section. Section 4 presents regularisation strategies which enable to obtain convex multi-variable isochoric invariants via modifications to a priori non-convex multi-variable isochoric invariants. Section 6 presents some challenging numerical examples in nearly and truly incompressible scenarios in order to provide an exhaustive comparison of the different mixed variational principles presented in Section 3 using a variety of Finite Element spaces. Finally, Section 7 provides some concluding remarks and a summary of the key contributions of this paper.

Three appendices have been included for the sake of completeness. Appendix A presents the relationship between the components of the tangent operators for the internal energy and those for its extended representation in terms of the arguments which define multi-variable convexity. Appendix $\mathrm{B}$ shows the relationship between the constitutive tensors arising from the classical Helmholtz's energy functional and those emerging from the internal energy. Finally, Appendix C expands on the developments of Section 4 including some tedious but necessary algebraic manipulations. 


\section{Nonlinear continuum electromechanics}

\subsection{Motion and deformation}

Let us consider the motion of a continuum representing a dielectric elastomer, defined by a domain $V$ with boundary $\partial V$ in its initial or material configuration. After the motion, the continuum occupies a spatial configuration defined by a domain $v$ with boundary $\partial v$. The motion is defined by a pseudo-time $t$ dependent mapping field $\phi$ which links a material particle from material configuration $\boldsymbol{X} \in V$ to spatial configuration $\boldsymbol{x} \in v$ according to $\boldsymbol{x}=\boldsymbol{\phi}(\boldsymbol{X}, t)$. Displacement boundary conditions can be defined as $\boldsymbol{x}=(\boldsymbol{\phi})_{\partial_{u} V}$ on the boundary $\partial_{u} V \subset \partial V$.

The deformation gradient tensor or fibre-map, defined as the material gradient of the spatial configuration $\boldsymbol{F}=\boldsymbol{\nabla}_{0} \boldsymbol{x}=\frac{\partial \boldsymbol{\phi}(\boldsymbol{X}, t)}{\partial \boldsymbol{X}}[29,30,33-35,52$, 83-86], the Jacobian or volume-map of the deformation $J=\operatorname{det} \boldsymbol{F}$ and the cofactor or area-map $\boldsymbol{H}=J \boldsymbol{F}^{-T}[46,52,57]$, relate differential fibre, area and volume elements, respectively, between material and spatial configurations (refer to Figure 1).

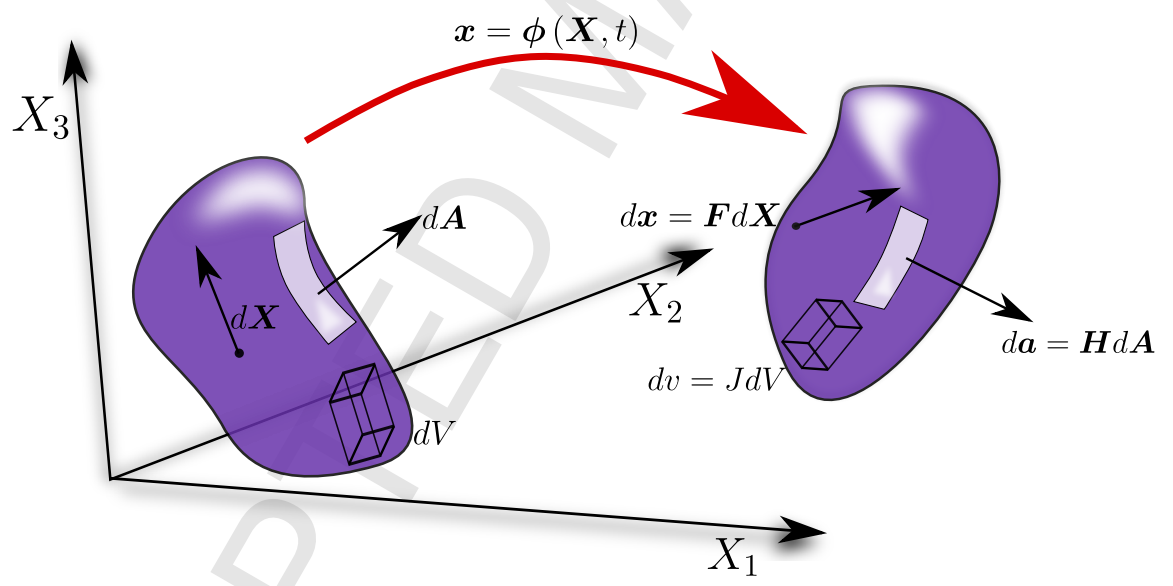

Figure 1: Deformation mapping of a continuum and definition of $\boldsymbol{F}, \boldsymbol{H}, J$. Material or Lagrangian infinitesimal fibre, area and volume elements $d \boldsymbol{X}$, $d \boldsymbol{A}$ and $d V$ are related accordingly to their spatial or Eulerian counterparts, namely $d \boldsymbol{x}, d \boldsymbol{a}$ and $d v$, respectively.

An alternative and algebraically more convenient definition of both the area and volume maps in terms of the tensor cross product operation $\mathbf{x}$ 
introduced in [81] and exploited in $[46,51,82]$ is given as

$$
\boldsymbol{H}=\frac{1}{2} \boldsymbol{F} \times \boldsymbol{F} ; \quad J=\frac{1}{3} \boldsymbol{H}: \boldsymbol{F},
$$

where for two-point second order tensors $\boldsymbol{A}$ and $\boldsymbol{B}$, the tensor cross operation $\mathbf{X}$ is computed as $(\boldsymbol{A} \times \boldsymbol{B})_{i I}=\mathcal{E}_{i j k} \mathcal{E}_{I J K} A_{j J} B_{k K}$, with $\mathcal{E}$ the third order alternating tensor. Throughout the paper, the symbol (.) is used to indicate the scalar product or contraction of a single index $\boldsymbol{a} \cdot \boldsymbol{b}=a_{i} b_{i}$; the symbol (:) is used to indicate double contraction of two indices $\boldsymbol{A}: \boldsymbol{B}=A_{i j} B_{i j}$; the symbol $(\times)$ is used to indicate the cross product between vectors $[\boldsymbol{a} \times \boldsymbol{b}]_{i}=$ $\mathcal{E}_{i j k} a_{j} b_{k}$ and the symbol $(\otimes)$ is used to indicate the outer or dyadic product $[\boldsymbol{a} \otimes \boldsymbol{b}]_{i j}=a_{i} b_{j}$.

Let us define $\delta \boldsymbol{u}$ and $\boldsymbol{u}$ as virtual and incremental variations of $\boldsymbol{x}$, which satisfy compatible displacement based boundary conditions on $\partial_{u} V$. With the help of the tensor cross product operation $\mathbf{x}$ introduced above, the first and second directional derivatives of the co-factor $\boldsymbol{H}$ and the Jacobian $J$ with respect to virtual and incremental variations of the geometry are evaluated as

$$
\begin{aligned}
D \boldsymbol{H}[\delta \boldsymbol{u}] & =\boldsymbol{F} \times \boldsymbol{\nabla}_{0} \delta \boldsymbol{u} ; & D^{2} \boldsymbol{H}[\delta \boldsymbol{u} ; \boldsymbol{u}] & =\nabla_{0} \delta \boldsymbol{u} \times \boldsymbol{\nabla}_{0} \boldsymbol{u} \\
D J[\delta \boldsymbol{u}] & =\boldsymbol{H}: \boldsymbol{\nabla}_{0} \delta \boldsymbol{u} ; & D^{2} J[\delta \boldsymbol{u} ; \boldsymbol{u}] & =\boldsymbol{F}:\left(\boldsymbol{\nabla}_{0} \delta \boldsymbol{u} \times \boldsymbol{\nabla}_{0} \boldsymbol{u}\right) .
\end{aligned}
$$

\subsection{Governing equations in nonlinear electromechanics}

The objective of this section is to present the governing equations which dictate the behaviour of dielectric elastomers, namely the Gauss and Faraday laws and the conservation of linear and angular momentum equations. For simplicity, the consideration of surrounding vacuum effects will be neglected in this work. The reader is referred to Reference [1] for the more general case where vacuum effects are not disregarded. 


\subsubsection{Gauss and Faraday laws}

The local version of the Gauss law in a Lagrangian setting can be written $\mathrm{as}^{3}$

$$
\begin{array}{ll}
\operatorname{DIV} \boldsymbol{D}_{0}=\rho_{0}^{e} & \text { in } V \\
\boldsymbol{D}_{0} \cdot \boldsymbol{N}=-\omega_{0}^{e} & \text { on } \partial_{\omega} V
\end{array}
$$

where $\rho_{0}^{e}$ represents an electric volume charge per unit of undeformed volume applied on the dielectric elastomer described in Section 2.1 in its material configuration $V, \omega_{0}^{e}$ is an electric surface charge per unit of undeformed area applied on $\partial_{\omega} V \subset \partial V$ and $\boldsymbol{D}_{0}$ is the Lagrangian electric displacement vector. Alternatively, equation (3) can be presented in a spatial setting in terms of the Eulerian electric displacement field $\boldsymbol{D}$, defined as $\boldsymbol{D}_{0}=\boldsymbol{H}^{T} \boldsymbol{D}[11,12]$. Analogously, the local form of the static Faraday law and its associated boundary conditions can be written as

$$
\begin{aligned}
\boldsymbol{E}_{0}=-\boldsymbol{\nabla}_{0} \varphi & \text { in } V ; \\
\varphi=(\varphi)_{\partial_{\varphi} V} & \text { on } \partial_{\varphi} V,
\end{aligned}
$$

where $\boldsymbol{E}_{0}$ is the Lagrangian electric field vector and $\varphi$ an electric potential field. In equation (4b), $\partial_{\varphi} V \subset \partial V$ represents the part of the boundary subjected to electric potential boundary conditions, such that $\partial_{\omega} V \cup \partial_{\varphi} V=$ $\partial V$ and $\partial_{\omega} V \cap \partial_{\varphi} V=\emptyset$. Equations (4) could alternatively be presented in a spatial or Eulerian setting in terms of the Eulerian electric field $\boldsymbol{E}$, related to its Lagrangian counterpart $\boldsymbol{E}_{0}$ via the standard relationship $\boldsymbol{E}_{0}=\boldsymbol{F}^{T} \boldsymbol{E}$ $[11,12]$.

\footnotetext{
${ }^{3}$ For the more general case where the effect of the surrounding vacuum cannot be neglected (as in Micro-Electro-Mechanical systems (MEMs) [21]), equation (3b) needs to account for the jump of the variable $\boldsymbol{D}_{0}$ across the boundary of the dielectric elastomer (refer to References [1] and [2]).
} 


\subsubsection{Translational and rotational equilibrium}

In the absence of inertial effects, the local conservation of linear momentum and its associated boundary conditions yield ${ }^{4}[52,86]$

$$
\begin{aligned}
\operatorname{DIV} \boldsymbol{P}+\boldsymbol{f}_{0} & =\mathbf{0} & & \text { in } V ; \\
\boldsymbol{P} \boldsymbol{N} & =\boldsymbol{t}_{0} & & \text { on } \partial_{t} V ; \\
\boldsymbol{\phi} & =(\boldsymbol{\phi})_{\partial_{u} V} & & \text { on } \partial_{u} V
\end{aligned}
$$

where $\boldsymbol{f}_{0}$ represents a body force per unit of undeformed volume in $V, \boldsymbol{t}_{0}$ a traction force per unit of undeformed area $\boldsymbol{t}_{0}$ on $\partial_{t} V \subset \partial V$, such that $\partial_{t} V \cup \partial_{u} V=\partial V$ and $\partial_{t} V \cap \partial_{u} V=\emptyset$ and $\boldsymbol{P}$, the first Piola-Kirchoff stress tensor. Furthermore, satisfaction of rotational equilibrium leads to the wellknown tensor condition $\boldsymbol{P} \boldsymbol{F}^{T}=\boldsymbol{F} \boldsymbol{P}^{T}[52]$.

\subsection{The internal energy density: multi-variable convexity in incompressible} electromechanics

For the closure of the system of equations defined by (3) and (5), an additional constitutive law satisfying appropriate constitutive inequalities [30] is needed. The most well accepted constitutive inequality, namely ellipticity, is automatically satisfied if the internal energy density functional $e$ per unit of undeformed volume $e=e\left(\boldsymbol{\nabla}_{0} \boldsymbol{x}, \boldsymbol{D}_{0}\right)$ is defined as [1]

$$
e\left(\boldsymbol{\nabla}_{0} \boldsymbol{x}, \boldsymbol{D}_{0}\right)=W\left(\boldsymbol{F}, \boldsymbol{H}, J, \boldsymbol{D}_{0}, \boldsymbol{d}\right) ; \quad \boldsymbol{d}=\boldsymbol{F} \boldsymbol{D}_{0},
$$

where $W$ represents a convex muti-variable functional in terms of the extended set of arguments $\mathcal{V}=\left\{\boldsymbol{F}, \boldsymbol{H}, J, \boldsymbol{D}_{0}, \boldsymbol{d}\right\}$.

In the context of truly or nearly incompressible scenarios, it is customary to additively decompose the internal energy into its isochoric and volumetric components (see References [52, 87-89]) $\hat{e}$ and $U$, respectively as

$e\left(\boldsymbol{\nabla}_{0} \boldsymbol{x}, \boldsymbol{D}_{0}\right)=\hat{e}\left(\boldsymbol{\nabla}_{0} \boldsymbol{x}, \boldsymbol{D}_{0}\right)+U\left(\operatorname{det} \boldsymbol{\nabla}_{0} \boldsymbol{x}\right) ; \quad \hat{e}\left(\boldsymbol{\nabla}_{0} \boldsymbol{x}, \boldsymbol{D}_{0}\right)=e\left(\left(\operatorname{det} \boldsymbol{\nabla}_{0} \boldsymbol{x}\right)^{-1 / 3} \boldsymbol{\nabla}_{0} \boldsymbol{x}, \boldsymbol{D}_{0}\right)$.

The first term on the left hand side of equation (7) leads to the deviatoric component of the first Piola-Kirchhoff tensor and the derivative of the function $U$ accounts for the pressure $p$. In the context of convex multi-variable

\footnotetext{
${ }^{4}$ For the more general case where the effect of the surrounding vacuum cannot be neglected, equation (5) needs to account for the jump of the variable $\boldsymbol{P}$ across the boundary of the dielectric elastomer (refer to References [1] and [2]).
} 
electro-mechanics, it is also possible to construct a similar decomposition to that in (7) as

$$
W\left(\boldsymbol{F}, \boldsymbol{H}, J, \boldsymbol{D}_{0}, \boldsymbol{d}\right)=\hat{W}\left(\boldsymbol{F}, \boldsymbol{H}, J, \boldsymbol{D}_{0}, \boldsymbol{d}\right)+U(J) .
$$

Following the ideas in References [46, 82, 87-89] in the context of incompressible elasticity, a simple way to ensure the correctness of the above additive decomposition (8), or equivalently, that only the $U$-term accounts for the pressure contribution, would be to construct $\hat{W}$ in terms of the isochoric components of $\boldsymbol{F}, \boldsymbol{H}$ and in terms of the spatial vector $\hat{\boldsymbol{d}}$, that is

$$
\hat{W}\left(\boldsymbol{F}, \boldsymbol{H}, J, \boldsymbol{D}_{0}, \boldsymbol{d}\right)=W\left(\hat{\boldsymbol{F}}, \hat{\boldsymbol{H}}, 1, \boldsymbol{D}_{0}, \hat{\boldsymbol{d}}\right),
$$

where the isochoric components of $\boldsymbol{F}$ and $\boldsymbol{H}[52]$ and the vector $\hat{\boldsymbol{d}}$ are defined as

$$
\hat{\boldsymbol{F}}=J^{-1 / 3} \boldsymbol{F} ; \quad \hat{\boldsymbol{H}}=J^{-2 / 3} \boldsymbol{H} ; \quad \hat{\boldsymbol{d}}=J^{-1 / 3} \boldsymbol{d} .
$$

As shown in Reference [1], the convex multi-variable nature of the internal energy $W$ (6) enables a one-to-one and invertible relationship between the elements of the extended set $\mathcal{V}$ and those in the associated set of work conjugates $\Sigma_{\mathcal{V}}=\left\{\boldsymbol{\Sigma}_{\boldsymbol{F}}, \boldsymbol{\Sigma}_{\boldsymbol{H}}, \Sigma_{J}, \boldsymbol{\Sigma}_{\boldsymbol{D}_{0}}, \boldsymbol{\Sigma}_{\boldsymbol{d}}\right\}$, defined as [1]

$$
\boldsymbol{\Sigma}_{\boldsymbol{F}}=\frac{\partial \hat{W}}{\partial \boldsymbol{F}} ; \quad \boldsymbol{\Sigma}_{\boldsymbol{H}}=\frac{\partial \hat{W}}{\partial \boldsymbol{H}} ; \quad \Sigma_{J}=\frac{\partial \hat{W}}{\partial J}+p ; \quad \boldsymbol{\Sigma}_{\boldsymbol{D}_{0}}=\frac{\partial \hat{W}}{\partial \boldsymbol{D}_{0}} ; \quad \boldsymbol{\Sigma}_{\boldsymbol{d}}=\frac{\partial \hat{W}}{\partial \boldsymbol{d}},
$$

where the pressure is related exclusively to the volumetric functional as $p=$ $U^{\prime}(J)$. As shown in References [1], it is possible to express the first PiolaKirchhoff stres tensor $\boldsymbol{P}$ and the material electric field $\boldsymbol{E}_{0}$ in terms of the arguments of the extended set $\Sigma_{\mathcal{V}}$ as

$$
\boldsymbol{P}=\boldsymbol{\Sigma}_{\boldsymbol{F}}+\boldsymbol{\Sigma}_{\boldsymbol{H}} \boldsymbol{x} \boldsymbol{F}+\Sigma_{J} \boldsymbol{H}+\boldsymbol{\Sigma}_{\boldsymbol{d}} \otimes \boldsymbol{D}_{0} ; \quad \boldsymbol{E}_{0}=\boldsymbol{\Sigma}_{\boldsymbol{D}_{0}}+\boldsymbol{F}^{T} \boldsymbol{\Sigma}_{\boldsymbol{d}}
$$

Alternatively, the first Piola-Kirchhoff stress tensor can be additively split into its deviatoric and volumetric components $\hat{\boldsymbol{P}}$ and $\boldsymbol{P}_{v}$, respectively, defined as

$$
\hat{\boldsymbol{P}}=\boldsymbol{\Sigma}_{\boldsymbol{F}}+\boldsymbol{\Sigma}_{\boldsymbol{H}} \times \boldsymbol{F}+\frac{\partial \hat{W}}{\partial J} \boldsymbol{H}+\boldsymbol{\Sigma}_{\boldsymbol{d}} \otimes \boldsymbol{D}_{0} ; \quad \boldsymbol{P}_{v}=p \boldsymbol{H}
$$


Following Reference [1], an expression for the Kirchhoff stress tensor and the spatial electric field (needed for post-processing purposes) emerges as

$$
\boldsymbol{\tau}=\boldsymbol{\Sigma}_{\boldsymbol{F}} \boldsymbol{F}^{T}+\left(\boldsymbol{\Sigma}_{\boldsymbol{H}} \boldsymbol{H}^{T}\right) \times \boldsymbol{I}+J \Sigma_{J} \boldsymbol{I}+\boldsymbol{\Sigma}_{\boldsymbol{d}} \otimes \boldsymbol{d} ; \quad \boldsymbol{E}=\boldsymbol{F}^{-T} \boldsymbol{\Sigma}_{\boldsymbol{D}_{0}}+\boldsymbol{\Sigma}_{\boldsymbol{d}}
$$

where $\boldsymbol{I}$ denotes the second order identity tensor.

As an example, let us consider the case of an ideal dielectric elastomer where the purely mechanical part of the internal energy is described via a Mooney-Rivlin material. The electro-mechanical internal energy representing this material based on an additive decomposition between isochoric and volumetric components can be defined as

$$
\hat{W}(\boldsymbol{F}, \boldsymbol{H}, J, \boldsymbol{d})=\eta J^{-2 / 3} I I_{\boldsymbol{F}}+\gamma J^{-2} I I_{\boldsymbol{H}}^{3 / 2}+\frac{I I_{\boldsymbol{d}}}{2 \varepsilon} J^{-2 / 3},
$$

where $I I_{(\bullet)}$ denotes the squared of the $L_{2}$ norm of the entity $(\bullet), \eta$ and $\gamma$ are two positive material parameters with units of $N / \mathrm{m}^{2}$ and $\varepsilon$, the electric permittivity of the material, related to that of the vacuum $\varepsilon_{0}=8,854 \times$ $10^{-12} \mathrm{~A}^{2} \mathrm{~s}^{4} \mathrm{~kg}^{-1} \mathrm{~m}^{-3}$ and the dimensionless relative permittivity $\varepsilon_{r}$ via the classical relationship $\varepsilon=\varepsilon_{r} \varepsilon_{0}$. Finally, the most commonly used expression for the volumetric strain energy component $U(J)$ is given by

$$
U(J)=\frac{\kappa}{2}(J-1)^{2},
$$

where $\kappa$ represents the bulk modulus of the material. In the context of convex multi-variable electromechanics, the volumetric term, exclusively depending upon the Jacobian $J$, must be a convex function, as that in equation (16).

\subsection{Tangent electromechanics operator for the internal energy}

With a Newton-Raphson type of solution process in mind, the internal energy $e=e\left(\boldsymbol{\nabla}_{0} \boldsymbol{x}, \boldsymbol{D}_{0}\right)$ can be further linearised leading to the tangent operator

$$
D^{2} e\left[\delta \boldsymbol{u}, \delta \boldsymbol{D}_{0} ; \boldsymbol{u}, \Delta \boldsymbol{D}_{0}\right]=D^{2} \hat{e}\left[\delta \boldsymbol{u}, \delta \boldsymbol{D}_{0} ; \boldsymbol{u}, \Delta \boldsymbol{D}_{0}\right]+D^{2} U(J)[\delta \boldsymbol{u} ; \boldsymbol{u}],
$$

where the tangent operator of both isochoric and volumetric components of the internal energy can be defined as

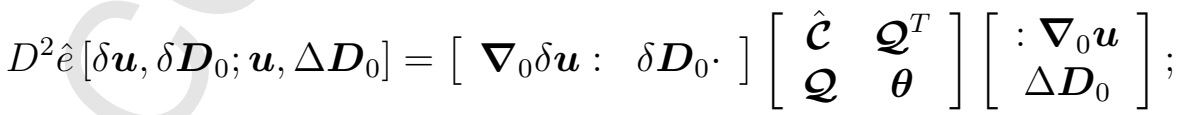

$$
\begin{aligned}
& D^{2} U[\delta \boldsymbol{u} ; \boldsymbol{u}]=\nabla_{0} \delta \boldsymbol{u}: \mathcal{C}_{p}: \nabla_{0} \boldsymbol{u},
\end{aligned}
$$


with the deviatoric and volumetric components of the fourth order elasticity tensor $\hat{\mathcal{C}}$ and $\mathcal{C}_{p}$, the third order piezoelectric tensor $\mathcal{Q}$ and the second order dielectric tensor $\boldsymbol{\theta}$ defined as

$$
\begin{array}{ll}
\hat{\mathcal{C}}=\left.\frac{\partial^{2} \hat{e}\left(\boldsymbol{F}, \boldsymbol{D}_{0}\right)}{\partial \boldsymbol{F} \partial \boldsymbol{F}}\right|_{\boldsymbol{F}=\boldsymbol{\nabla}_{0} \boldsymbol{x}} ; & \mathcal{C}_{p}=\left.\frac{\partial^{2} U(\boldsymbol{F})}{\partial \boldsymbol{F} \partial \boldsymbol{F}}\right|_{\boldsymbol{F}=\boldsymbol{\nabla}_{0} \boldsymbol{x}} ; \\
\mathcal{Q}=\left.\frac{\partial^{2} \hat{e}\left(\boldsymbol{F}, \boldsymbol{D}_{0}\right)}{\partial \boldsymbol{D}_{0} \partial \boldsymbol{F}}\right|_{\boldsymbol{F}=\boldsymbol{\nabla}_{0} \boldsymbol{x}} ; & \boldsymbol{\theta}=\left.\frac{\partial^{2} \hat{e}\left(\boldsymbol{F}, \boldsymbol{D}_{0}\right)}{\partial \boldsymbol{D}_{0} \partial \boldsymbol{D}_{0}}\right|_{\boldsymbol{F}=\boldsymbol{\nabla}_{0} \boldsymbol{x}} .
\end{array}
$$

With the help of the tensor cross product [1, 46, 81], a more physically insightful representation of the tangent operator of the isochoric and volumetric components of the internal energy (18) is obtained as

$$
\begin{aligned}
D^{2} \hat{e}\left[\delta \boldsymbol{u}, \delta \boldsymbol{D}_{0} ; \boldsymbol{u}, \Delta \boldsymbol{D}_{0}\right] & =\left[\mathbb{S}_{\delta}\right]^{T}\left[\mathbb{H}_{\hat{W}}\right]\left[\mathbb{S}_{\Delta}\right]+\left(\boldsymbol{\Sigma}_{\boldsymbol{H}}+\Sigma_{J} \boldsymbol{F}\right):\left(\boldsymbol{\nabla}_{0} \delta \boldsymbol{u} \times \boldsymbol{\nabla}_{0} \boldsymbol{u}\right) \\
& +\boldsymbol{\Sigma}_{\boldsymbol{d}} \cdot\left(\left(\boldsymbol{\nabla}_{0} \delta \boldsymbol{u}\right) \Delta \boldsymbol{D}_{0}+\left(\boldsymbol{\nabla}_{0} \boldsymbol{u}\right) \delta \boldsymbol{D}_{0}\right) \\
D^{2} U[\delta \boldsymbol{u} ; \boldsymbol{u}] & =U^{\prime \prime}(J)\left(\boldsymbol{H}: \boldsymbol{\nabla}_{0} \delta \boldsymbol{u}\right)\left(\boldsymbol{H}: \boldsymbol{\nabla}_{0} \boldsymbol{u}\right)+U^{\prime}(J) \boldsymbol{F}:\left(\boldsymbol{\nabla}_{0} \delta \boldsymbol{u} \times \boldsymbol{\nabla}_{0} \boldsymbol{u}\right),
\end{aligned}
$$

where

$$
\begin{aligned}
& {\left[\mathbb{S}_{\delta}\right]^{T}=} {\left[\begin{array}{lll}
\left(\boldsymbol{\nabla}_{0} \delta \boldsymbol{u}\right):\left(\boldsymbol{\nabla}_{0} \delta \boldsymbol{u} \times \boldsymbol{F}\right):\left(\boldsymbol{\nabla}_{0} \delta \boldsymbol{u}: \boldsymbol{H}\right) & \delta \boldsymbol{D}_{0} \cdot( & \left.\left(\left(\boldsymbol{\nabla}_{0} \delta \boldsymbol{u}\right) \boldsymbol{D}_{0}+\boldsymbol{F} \delta \boldsymbol{D}_{0}\right) \cdot\right]
\end{array}\right] } \\
& {\left[\mathbb{S}_{\Delta}\right]=\left[\begin{array}{c}
:\left(\boldsymbol{\nabla}_{0} \boldsymbol{u}\right) \\
:\left(\boldsymbol{F} \times \boldsymbol{\nabla}_{0} \boldsymbol{u}\right) \\
\left(\boldsymbol{H}: \boldsymbol{\nabla}_{0} \boldsymbol{u}\right) \\
\Delta \boldsymbol{D}_{0} \\
\boldsymbol{\nabla}_{0} \boldsymbol{u} \boldsymbol{D}_{0}+\boldsymbol{F} \Delta \boldsymbol{D}_{0}
\end{array}\right] }
\end{aligned}
$$

and with the extended Hessian operator $\left[\mathbb{H}_{\hat{W}}\right]$ denoting the symmetric positive definite operator containing the second derivatives of $\hat{W}\left(\boldsymbol{F}, \boldsymbol{H}, J, \boldsymbol{D}_{0}, \boldsymbol{d}\right)$ as

$$
\left[\mathbb{H}_{\hat{W}}\right]=\left[\begin{array}{ccccc}
\hat{W}_{\boldsymbol{F} \boldsymbol{F}} & \hat{W}_{\boldsymbol{F H}} & \hat{W}_{\boldsymbol{F} J} & \hat{W}_{\boldsymbol{F} \boldsymbol{D}_{0}} & \hat{W}_{\boldsymbol{F d}} \\
\hat{W}_{\boldsymbol{H} \boldsymbol{F}} & \hat{W}_{\boldsymbol{H} \boldsymbol{H}} & \hat{W}_{\boldsymbol{H} J} & \hat{W}_{\boldsymbol{H} \boldsymbol{D}_{0}} & \hat{W}_{\boldsymbol{H} \boldsymbol{d}} \\
\hat{W}_{J \boldsymbol{F}} & \hat{W}_{J \boldsymbol{H}} & \hat{W}_{J J} & \hat{W}_{J \boldsymbol{D}_{0}} & \hat{W}_{J \boldsymbol{d}} \\
\hat{W}_{\boldsymbol{D}_{0} \boldsymbol{F}} & \hat{W}_{\boldsymbol{D}_{0} \boldsymbol{H}} & \hat{W}_{\boldsymbol{D}_{0} J} & \hat{W}_{\boldsymbol{D}_{0} \boldsymbol{D}_{0}} & \hat{W}_{\boldsymbol{D}_{0} \boldsymbol{d}} \\
\hat{W}_{\boldsymbol{d} \boldsymbol{F}} & \hat{W}_{\boldsymbol{d H}} & \hat{W}_{\boldsymbol{d} J} & \hat{W}_{\boldsymbol{d \boldsymbol { D } _ { 0 }}} & \hat{W}_{\boldsymbol{d d}}
\end{array}\right] .
$$


As shown in Appendix A, it is possible to relate the constitutive tensors $\hat{\mathcal{C}}, \boldsymbol{Q}$ and $\boldsymbol{\theta}$ in equation (19) to the components of the Hessian operator $\left[\mathbb{H}_{\hat{W}}\right]$ in (22). Moreover, the volumetric component of the elasticity tensor $\mathcal{C}_{p}$ (19) can be related to the first and second derivatives of $U(J)$, as shown in Appendix A.

Extending the ideas presented in Reference [1], positive definiteness of the Hessian operator $\left[\mathbb{H}_{\hat{W}}\right]$ in above $(22)$ and positiveness of $U^{\prime \prime}(J)$ in $(20)_{b}$ extends the concept of ellipticity (rank-one convexity) to the field of electromechanics for the specific case of energy functionals additively decomposed into isochoric and volumetric components. As shown in Reference [3], the authors show that this condition has very strong physical implications as it guarantees the existence of physical wave speeds (Legendre-Hadamard condition [30]).

For instance, for the constitutive model defined in equation (15), the Hessian operator $\left[\mathbb{H}_{\hat{W}}\right]$ associated to the isochoric component of the internal energy $\hat{W}$ is defined as

$$
\left[\mathbb{H}_{\hat{W}}\right]=\left[\begin{array}{ccccc}
\frac{2 \eta}{J^{2 / 3}} \boldsymbol{I} & \mathbf{0} & -\frac{4 \eta}{3 J^{5 / 3}} \boldsymbol{F} & \mathbf{0} & \mathbf{0} \\
\mathbf{0} & \hat{W}_{\boldsymbol{H} \boldsymbol{H}} & -\frac{6 \gamma}{J^{3}} I I_{\boldsymbol{H}}^{1 / 2} \boldsymbol{H} & \mathbf{0} & \mathbf{0} \\
-\frac{4 \eta}{3 J^{5 / 3}} \boldsymbol{F} & -\frac{6 \gamma}{J^{3}} I I_{\boldsymbol{H}}^{1 / 2} \boldsymbol{H} & \hat{W}_{J J} & \mathbf{0} & -\frac{2}{3 \varepsilon J^{5 / 3}} \boldsymbol{d} \\
\mathbf{0} & \mathbf{0} & \mathbf{0} & \mathbf{0} & \mathbf{0} \\
\mathbf{0} & \mathbf{0} & -\frac{2}{3 \varepsilon J^{5 / 3}} \boldsymbol{d} & \mathbf{0} & \frac{1}{\varepsilon J^{2 / 3}} \boldsymbol{I}
\end{array}\right],
$$

where $\mathcal{I}$ denotes the fourth order identity tensor, i.e, $\mathcal{I}_{i I j J}=\delta_{i j} \delta_{I J}$ and with the slightly more convoluted terms $\hat{W}_{\boldsymbol{H} \boldsymbol{H}}$ and $\hat{W}_{J J}$, denoting the second derivatives of $\hat{W}$ with respect to $\boldsymbol{H}$ and $J$, respectively, defined as

$$
\begin{aligned}
\hat{W}_{\boldsymbol{H} \boldsymbol{H}} & =\frac{3 \gamma}{J^{2}} I I_{\boldsymbol{H}}^{1 / 2} \mathcal{I}+\frac{3 \gamma}{J^{2}} I I_{\boldsymbol{H}}^{-1 / 2} \boldsymbol{H} \otimes \boldsymbol{H} \\
\hat{W}_{J J} & =\frac{10 \eta}{9 J^{8 / 3}} I I_{\boldsymbol{F}}+\frac{6 \gamma}{J^{4}} I I_{\boldsymbol{H}}^{3 / 2}+\frac{5}{9 \varepsilon J^{8 / 3}} I I_{\boldsymbol{d}} .
\end{aligned}
$$

Positive definiteness of the exclusively mechanical contribution of the Hessian operator $\left[\mathbb{H}_{\hat{W}}\right]$ defined in equations (23) and (24) has been proved in Reference [40]. For completeness, we show positive definiteness of the electromechanical contribution of $\left[\mathbb{H}_{\hat{W}}\right]$, associated to the electromechanical energy component (15) $\hat{W}_{e m}$ of $\hat{W}$ (refer to (15)), defined as

$$
\hat{W}_{e m}=\frac{I I_{\boldsymbol{d}}}{2 \varepsilon} J^{-2 / 3}
$$


Proof of positive definiteness of the Hessian operator of the above electromechanical energy functional $\hat{W}_{e m}$, namely $\left[\mathbb{H}_{\hat{W}_{e m}}\right]$, follows from the following inequality

$$
\left[\begin{array}{l}
\delta \boldsymbol{d} \\
\delta J
\end{array}\right]^{T}\left[\mathbb{H}_{\hat{W}_{e m}}\right]\left[\begin{array}{l}
\delta \boldsymbol{d} \\
\delta J
\end{array}\right]=\frac{1}{\varepsilon} I I_{\boldsymbol{g}}+\frac{1}{9 \varepsilon}\left(\frac{\delta J}{J}\right)^{2} I I_{\hat{\boldsymbol{d}}} \geq 0
$$

where the vector $\boldsymbol{g}$ in above equation (26) is defined as $\boldsymbol{g}=\delta \hat{\boldsymbol{d}}-\sqrt{\frac{2}{3}} \frac{\delta J}{J} \hat{\boldsymbol{d}}$, with $\hat{\boldsymbol{d}}$ defined in equation (10) and with $\delta \hat{\boldsymbol{d}}=J^{-1 / 3} \delta \boldsymbol{d}$.

Finally, notice that the fourth row and column of the Hessian operator $\left[\mathbb{H}_{\hat{W}}\right]$ in above equation (23), associated to the derivatives of the energy functional with respect to $\boldsymbol{D}_{0}$, vanish. This is typical for the case of an ideal dielectric elastomer. More complex constitutive models incorporating electrostriction effects add contributions to this column and row, as shown in References [1, 3].

\subsection{The Hemlholtz's energy density functional}

The definition of multi-variable convexity in (6) ensures a one to one relationship between the variables $\boldsymbol{D}_{0}$ and $-\boldsymbol{\nabla}_{0} \varphi$. In this case, it is possible to define an alternative energy functional to the internal energy $e=e\left(\boldsymbol{\nabla}_{0} \boldsymbol{x}, \boldsymbol{D}_{0}\right)$ called the Helmholtz's energy funtional $\Phi=\Phi\left(\boldsymbol{\nabla}_{0} \boldsymbol{x},-\boldsymbol{\nabla}_{0} \varphi\right)$, by making use of the following Legendre transform $\mathrm{as}^{5}$.

$$
\Phi\left(\boldsymbol{\nabla}_{0} \boldsymbol{x},-\boldsymbol{\nabla}_{0} \varphi\right)=-\sup _{\boldsymbol{D}_{0}}\left\{-\boldsymbol{\nabla}_{0} \varphi \cdot \boldsymbol{D}_{0}-\hat{e}\left(\boldsymbol{\nabla}_{0} \boldsymbol{x}, \boldsymbol{D}_{0}\right)-U\left(\operatorname{det} \boldsymbol{\nabla}_{0} \boldsymbol{x}\right)\right\} .
$$

The above Legendre transformation enables to additively decompose the Helmholtz's energy functional in terms of isochoric $\hat{\Phi}$ and volumetric $U$ components as

$$
\Phi\left(\boldsymbol{\nabla}_{0} \boldsymbol{x},-\boldsymbol{\nabla}_{0} \varphi\right)=\hat{\Phi}\left(\boldsymbol{\nabla}_{0} \boldsymbol{x},-\boldsymbol{\nabla}_{0} \varphi\right)+U\left(\operatorname{det} \boldsymbol{\nabla}_{0} \boldsymbol{x}\right),
$$

leading to a definition for the stress and electric fields as

$$
\hat{\boldsymbol{P}}=\left.\frac{\partial \hat{\Phi}\left(\boldsymbol{F}, \boldsymbol{E}_{0}\right)}{\partial \boldsymbol{F}}\right|_{\substack{\boldsymbol{F}=\boldsymbol{\nabla}_{0} \boldsymbol{x} \\ \boldsymbol{E}_{0}=-\boldsymbol{\nabla}_{0} \varphi}} ; \quad \boldsymbol{P}_{v}=\left.\frac{\partial U(\operatorname{det} \boldsymbol{F})}{\partial \boldsymbol{F}}\right|_{\substack{\boldsymbol{F}=\nabla_{0} \boldsymbol{x} \\ \boldsymbol{E}_{0}=-\nabla_{0} \varphi}} ; \quad \boldsymbol{D}_{0}=-\left.\frac{\partial \hat{\Phi}\left(\boldsymbol{F}, \boldsymbol{E}_{0}\right)}{\partial \boldsymbol{E}_{0}}\right|_{\substack{\boldsymbol{F}=\boldsymbol{\nabla}_{0} \boldsymbol{x} \\ \boldsymbol{E}_{0}=-\boldsymbol{\nabla}_{0} \varphi}},
$$

\footnotetext{
${ }^{5}$ An advantage of employing a constitutive model defined by the energy functional in (6) is that it ensures the existence of the Helmholtz's energy density ab initio, which cannot be necessarily guaranteed otherwise.
} 
with the first Piola-Kirchhoff stress tensor $\boldsymbol{P}$ additively decomposed into deviatoric and volumetric components, namely $\boldsymbol{P}=\hat{\boldsymbol{P}}+\boldsymbol{P}_{v}$.

\subsection{Tangent operators for the Helmholtz's energy functional}

The tangent operator for the Helmholtz's energy functional $\Phi\left(\boldsymbol{\nabla}_{0} \boldsymbol{x},-\boldsymbol{\nabla}_{0} \varphi\right)$ (27) and (28) can be additively decomposed as

$$
D^{2} \Phi\left[\delta \boldsymbol{u}, \delta \boldsymbol{D}_{0} ; \boldsymbol{u}, \Delta \boldsymbol{D}_{0}\right]=D^{2} \hat{\Phi}\left[\delta \boldsymbol{u}, \delta \boldsymbol{D}_{0} ; \boldsymbol{u}, \Delta \boldsymbol{D}_{0}\right]+D^{2} U(J)[\delta \boldsymbol{u} ; \boldsymbol{u}],
$$

where the tangent operators for the volumetric component $U(J)$ is identical to that presented in equations $(18)_{b}$ and $(20)_{b}$. The tangent operator for the isochoric contribution $\hat{\Phi}$ is computed as

$$
D^{2} \hat{\Phi}[\delta \boldsymbol{u}, \delta \varphi ; \boldsymbol{u}, \Delta \varphi]=\left[\boldsymbol{\nabla}_{0} \delta \boldsymbol{u}: \quad-\boldsymbol{\nabla}_{0} \delta \varphi \cdot\right]\left[\begin{array}{cc}
\hat{\mathcal{C}}^{*} & -\mathcal{P}^{T} \\
-\mathcal{P} & -\varepsilon
\end{array}\right]\left[\begin{array}{c}
: \nabla_{0} \boldsymbol{u} \\
-\nabla_{0} \Delta \varphi
\end{array}\right]
$$

with the deviatoric part of the fourth order elastic tensor $\hat{\mathcal{C}}^{*}$, the third order piezoelectric tensor $\mathcal{P}$ and the second order dielectric tensor $\boldsymbol{\varepsilon}$ defined as

$$
\hat{\mathcal{C}}^{*}=\left.\frac{\partial^{2} \hat{\Phi}\left(\boldsymbol{F}, \boldsymbol{E}_{0}\right)}{\partial \boldsymbol{F} \partial \boldsymbol{F}}\right|_{\substack{\boldsymbol{F}=\nabla_{0} \boldsymbol{x} \\ \boldsymbol{E}_{0}=-\nabla_{0} \varphi}} ; \mathcal{P}=-\left.\frac{\partial^{2} \hat{\Phi}\left(\boldsymbol{F}, \boldsymbol{E}_{0}\right)}{\partial \boldsymbol{F} \partial \boldsymbol{E}_{0}}\right|_{\substack{\boldsymbol{F}=\nabla_{0} \boldsymbol{x} \\ \boldsymbol{E}_{0}=-\nabla_{0} \varphi}} ; \boldsymbol{\varepsilon}=-\left.\frac{\partial^{2} \hat{\Phi}\left(\boldsymbol{F}, \boldsymbol{E}_{0}\right)}{\partial \boldsymbol{E}_{0} \partial \boldsymbol{E}_{0}}\right|_{\substack{\boldsymbol{F}=\nabla_{0} \boldsymbol{x} \\ \boldsymbol{E}_{0}=-\nabla_{0} \varphi}} .
$$

As shown in Appendix B, it is possible to relate the constitutive tensors $\hat{\mathcal{C}}^{\star}, \mathcal{P}$ and $\varepsilon$ in (32) to those emerging from the tangent operator of the internal energy, namely $\hat{\mathcal{C}}, \mathcal{Q}$ and $\boldsymbol{\theta}$ in (19) and hence, to the components of the Hessian operator $\left[\mathbb{H}_{\hat{W}}\right]$ in (22) (as the necessary relationships between $\hat{\mathcal{C}}$, $\mathcal{Q}$ and $\boldsymbol{\theta}$ and the components of $\left[\mathbb{H}_{\hat{W}}\right]$ are included in Appendix A).

\section{Variational formulations}

This Section presents a series of mixed variational principles and their finite element implementation for nearly and truly incompressible electroelasticity. The more standard four (and three) displacement-potential-Jacobianpressure [90] (and displacement-potential-pressure) [65] variational principles and new more sophisticated extended $\mathrm{Hu}$-Washizu mixed variational principles obtained following the ideas in Reference [1] will be presented in this Section. 
3.1. Standard displacement and electric potential based variational principle

Some authors [90] advocate for a four-field mixed variational principle where the geometry, the electric potential, the Jacobian and the pressure are the unknown variables, defined as

$$
\begin{aligned}
\Pi_{\Phi_{N I}}\left(\boldsymbol{x}^{*}, \varphi^{*}, \widetilde{J}^{*}, p^{*}\right) & =\inf _{\boldsymbol{x}, \widetilde{J}} \sup _{\varphi, p}\left\{\int_{V} \hat{\Phi}\left(\boldsymbol{\nabla}_{0} \boldsymbol{x},-\boldsymbol{\nabla}_{0} \varphi\right) d V+\int_{V} U(\widetilde{J}) d V\right. \\
& \left.+\int_{V} p\left(J_{\boldsymbol{x}}-\widetilde{J}\right) d V-\Pi_{e x t}(\boldsymbol{x}, \varphi)\right\},
\end{aligned}
$$

where $(\bullet)^{*}$ in above equation (33) indicates the exact solution of the variable $(\bullet)$ and

$$
\Pi_{e x t}(\boldsymbol{x}, \varphi)=\int_{V} \boldsymbol{f}_{0} \cdot \boldsymbol{x} d V+\int_{\partial_{t} V} \boldsymbol{t}_{0} \cdot \boldsymbol{x} d A-\int_{V} \rho_{0}^{e} \varphi d V-\int_{\partial_{\omega} V} \omega_{0}^{e} \varphi d A,
$$

the total external work due to the action of external mechanical forces and electric charges.

Alternatively, other authors [65] resort to a three-field mixed variational principle, in terms of the complementary energy of the volumetric energy functional $U(\tilde{J})$, defined as

$$
\begin{aligned}
\Pi_{\Phi_{N I C}}\left(\boldsymbol{x}^{*}, \varphi^{*}, p^{*}\right) & =\inf _{\boldsymbol{x}} \sup _{\varphi, p}\left\{\int_{V} \hat{\Phi}\left(\boldsymbol{\nabla}_{0} \boldsymbol{x},-\boldsymbol{\nabla}_{0} \varphi\right) d V+\int_{V} \Gamma(p) d V\right. \\
& \left.+\int_{V} p J_{\boldsymbol{x}} d V-\Pi_{e x t}(\boldsymbol{x}, \varphi)\right\},
\end{aligned}
$$

where $\Gamma(p)$ denotes the complementary energy of the volumetric functional $U(\widetilde{J})$, defined as ${ }^{6}$

$$
\Gamma(p)=\inf _{\widetilde{J}}\{U(\widetilde{J})-p \widetilde{J}\}
$$

${ }^{6}$ An explicit definition of the complementary energy for the volumetric functional $U(\widetilde{J})$ in equation (16) can be obtained as

$$
\Gamma(p)=-p\left(\frac{p}{2 \kappa}+1\right) .
$$


For fully incompressible materials $(\Gamma=-p)$, the above variational principle can be modified as

$$
\begin{aligned}
\Pi_{\Phi_{I}}\left(\boldsymbol{x}^{*}, \varphi^{*}, p^{*}\right) & =\inf _{\boldsymbol{x}} \sup _{\varphi}\left\{\int_{V} \hat{\Phi}\left(\boldsymbol{\nabla}_{0} \boldsymbol{x},-\nabla_{0} \varphi\right) d V+\int_{V} p\left(J_{\boldsymbol{x}}-1\right) d V\right. \\
& \left.-\Pi_{e x t}(\boldsymbol{x}, \varphi)\right\} .
\end{aligned}
$$

3.2. Extended Hu-Washizu mixed variational principle for nearly incompressible and fully incompressible scenarios

Gil and Ortigosa [1] presented a series of novel Hu-Washizu mixed variational principles in the context of compressible electro-elasticity. Based on that work [1], a novel enhanced $\mathrm{Hu}$-Washizu mixed variational principle, tailor-made for nearly and truly incompressible materials, where the electromechanical internal energy is additively decomposed as in Section 2.3, can be defined as

$$
\begin{aligned}
& \Pi_{W_{N I}}\left(\boldsymbol{x}^{*}, \boldsymbol{F}^{*}, \boldsymbol{H}^{*}, J^{*}, \boldsymbol{\Sigma}_{\boldsymbol{F}}^{*}, \boldsymbol{\Sigma}_{\boldsymbol{H}}^{*}, \hat{\Sigma}_{J}^{*}, \varphi^{*}, \boldsymbol{D}_{0}^{*}, \boldsymbol{d}^{*}, \boldsymbol{\Sigma}_{\boldsymbol{d}}^{*}, \widetilde{J}^{*}, p^{*}\right) \\
& =\inf _{\boldsymbol{x}, \boldsymbol{F}, \boldsymbol{H}, J, \boldsymbol{D}_{0}, \boldsymbol{d}, \widetilde{J}} \sup _{\boldsymbol{\Sigma}_{\boldsymbol{F}}, \boldsymbol{\Sigma}_{\boldsymbol{H}}, \hat{\Sigma}_{J}, \varphi, \boldsymbol{\Sigma}_{\boldsymbol{d}}, p}\left\{\int_{V} \hat{W}(\mathcal{V}) d V+\int_{V} U(\widetilde{J}) d V+\int_{V} \boldsymbol{D}_{0} \cdot \nabla_{0} \varphi d V\right. \\
& +\int_{V}\left[\boldsymbol{\Sigma}_{\boldsymbol{F}}:\left(\boldsymbol{F}_{\boldsymbol{x}}-\boldsymbol{F}\right)+\boldsymbol{\Sigma}_{\boldsymbol{H}}:\left(\boldsymbol{H}_{\boldsymbol{x}}-\boldsymbol{H}\right)+\hat{\Sigma}_{J}\left(J_{\boldsymbol{x}}-J\right)\right. \\
& \left.\left.+\boldsymbol{\Sigma}_{\boldsymbol{d}} \cdot\left(\boldsymbol{F}_{\boldsymbol{x}} \boldsymbol{D}_{0}-\boldsymbol{d}\right)+p\left(J_{\boldsymbol{x}}-\widetilde{J}\right)\right] d V-\Pi_{e x t}(\boldsymbol{x}, \varphi)\right\},
\end{aligned}
$$

where $\left\{\boldsymbol{F}_{\boldsymbol{x}}, \boldsymbol{H}_{\boldsymbol{x}}, J_{\boldsymbol{x}}\right\}$ in above equation (38) denote the geometrically compatible strain measures

$$
\boldsymbol{F}_{\boldsymbol{x}}=\boldsymbol{\nabla}_{0} \boldsymbol{x} ; \quad \boldsymbol{H}_{\boldsymbol{x}}=\frac{1}{2} \boldsymbol{\nabla}_{0} \boldsymbol{x} \times \boldsymbol{\nabla}_{0} \boldsymbol{x} ; \quad J_{\boldsymbol{x}}=\operatorname{det} \boldsymbol{\nabla}_{0} \boldsymbol{x} .
$$

Notice in the above variational principle, the definition of two Jacobian variables $J$ and $\widetilde{J}$ and their respective work conjugates $\hat{\Sigma}_{J}$ and $p$. This is not the case for the mixed variational principles presented in Reference $[1,2]$, where only the work conjugate $\Sigma_{J}$ is considered. This explicit distinction is the consequence of the additive split of the internal energy in terms of its isochoric and volumetric components $\hat{W}(\mathcal{V})$ and $U(J)$, which guarantees that the Lagrange multiplier $p$ coincides (in a week manner) with the hydrostatic pressure. 
Following the ideas presented in Reference [46], an alternative mixed variational principle where the field $\tilde{J}$ does no longer feature in the formulation can be derived as

$$
\begin{aligned}
& \Pi_{W_{N I C}}\left(\boldsymbol{x}^{*}, \boldsymbol{F}^{*}, \boldsymbol{H}^{*}, J^{*}, \boldsymbol{\Sigma}_{\boldsymbol{F}}^{*}, \boldsymbol{\Sigma}_{\boldsymbol{H}}^{*}, \hat{\Sigma}_{J}^{*}, \varphi^{*}, \boldsymbol{D}_{0}^{*}, \boldsymbol{d}^{*}, \boldsymbol{\Sigma}_{\boldsymbol{d}}^{*}, p^{*}\right) \\
& =\inf _{\boldsymbol{x}, \boldsymbol{F}, \boldsymbol{H}, J, \boldsymbol{D}_{0}, \boldsymbol{d}, \widetilde{J}} \sup _{\boldsymbol{\Sigma}_{\boldsymbol{F}}, \boldsymbol{\Sigma}_{\boldsymbol{H}}, \hat{\Sigma}_{J}, \varphi, \boldsymbol{\Sigma}_{\boldsymbol{d}}, p}\left\{\int_{V} \hat{W}(\mathcal{V}) d V+\int_{V} \Gamma(p) d V\right. \\
& +\int_{V} \boldsymbol{D}_{0} \cdot \boldsymbol{\nabla}_{0} \varphi d V+\int_{V}\left[\boldsymbol{\Sigma}_{\boldsymbol{F}}:\left(\boldsymbol{F}_{\boldsymbol{x}}-\boldsymbol{F}\right)+\boldsymbol{\Sigma}_{\boldsymbol{H}}:\left(\boldsymbol{H}_{\boldsymbol{x}}-\boldsymbol{H}\right)+\hat{\Sigma}_{J}\left(J_{\boldsymbol{x}}-J\right)\right. \\
& \left.\left.+\boldsymbol{\Sigma}_{\boldsymbol{d}} \cdot\left(\boldsymbol{F}_{\boldsymbol{x}} \boldsymbol{D}_{0}-\boldsymbol{d}\right)+p J_{\boldsymbol{x}}\right] d V-\Pi_{e x t}(\boldsymbol{x}, \varphi)\right\} .
\end{aligned}
$$

For fully incompressible materials $(\Gamma(p)=-p)$, the above variational principle $\Pi_{W_{N I}}$ (38) can be modified as

$$
\begin{aligned}
& \Pi_{W_{I}}\left(\boldsymbol{x}^{*}, \boldsymbol{F}^{*}, \boldsymbol{H}^{*}, J^{*}, \boldsymbol{\Sigma}_{\boldsymbol{F}}^{*}, \boldsymbol{\Sigma}_{\boldsymbol{H}}^{*}, \hat{\Sigma}_{J}^{*}, \varphi^{*}, \boldsymbol{D}_{0}^{*}, \boldsymbol{d}^{*}, \boldsymbol{\Sigma}_{\boldsymbol{d}}^{*}, p^{*}\right) \\
& =\inf _{\boldsymbol{x}, \boldsymbol{F}, \boldsymbol{H}, J, \boldsymbol{D}_{0}, \boldsymbol{d}} \sup _{\boldsymbol{\Sigma}_{\boldsymbol{F}}, \boldsymbol{\Sigma}_{\boldsymbol{H}}, \Sigma_{J}, \varphi, \boldsymbol{\Sigma}_{\boldsymbol{d}}, p}\left\{\int_{V} \hat{W}(\mathcal{V}) d V+\int_{V} \boldsymbol{D}_{0} \cdot \boldsymbol{\nabla}_{0} \varphi d V\right. \\
& +\int_{V}\left[\boldsymbol{\Sigma}_{\boldsymbol{F}}:\left(\boldsymbol{F}_{\boldsymbol{x}}-\boldsymbol{F}\right)+\boldsymbol{\Sigma}_{\boldsymbol{H}}:\left(\boldsymbol{H}_{\boldsymbol{x}}-\boldsymbol{H}\right)+\hat{\Sigma}_{J}\left(J_{\boldsymbol{x}}-J\right)\right. \\
& \left.\left.+\boldsymbol{\Sigma}_{\boldsymbol{d}} \cdot\left(\boldsymbol{F}_{\boldsymbol{x}} \boldsymbol{D}_{0}-\boldsymbol{d}\right)+p\left(J_{\boldsymbol{x}}-1\right)\right] d V-\Pi_{e x t}(\boldsymbol{x}, \varphi)\right\} .
\end{aligned}
$$

Notice that following a similar procedure to that in Reference [46], it would be possible to define two partial and a total Legendre-transformations of the isochoric internal energy functional $\hat{W}(\mathcal{V})$ in the above three mixed variational principles $\Pi_{W_{N I}}(38), \Pi_{W_{N I C}}$, (40) and $\Pi_{W_{I}}$ (41). This would lead to the definition of nine additional mixed variational principles (three for each of the aforementioned variational principles, namely $\Pi_{W_{N I}}(38), \Pi_{W_{N I C}}$, (40) and $\left.\Pi_{W_{I}}(41)\right)$ in terms of the extended Helmholtz's, Gibb's and enthalpy energy functionals (refer to Section 4.6 in Reference [46] for a definition of these energy functionals).

\section{Convexification (stabilisation) of materially unstable invariants}

For isotropic materials, the isochoric component of the extended internal energy $W$ is typically represented $[11,12,15]$ as

$$
\hat{W}\left(\boldsymbol{F}, \boldsymbol{H}, J, \boldsymbol{D}_{0}, \boldsymbol{d}\right)=\hat{w}\left(I I_{\boldsymbol{F}}, I I_{\boldsymbol{H}}, J, I I_{\boldsymbol{D}_{0}}, I I_{\boldsymbol{d}}, I I_{\boldsymbol{h}}\right),
$$


where $\boldsymbol{h}=\boldsymbol{H} \boldsymbol{D}_{0}$. Unfortunately, since $\boldsymbol{h}$ is not included in the extended set $\mathcal{V}$, the invariant $I I_{\boldsymbol{h}}$ is not convex multi-variable itself. Moreover, consideration of the specific convexity restrictions for the isochoric representation of the second argument in above equation (42), namely $I I_{\hat{\boldsymbol{H}}}$ (see Reference [40] in the context of pure elasticity and the second term on the right hand side of equation (15)) enables us to conclude that the isochoric component of the internal energy $\hat{W}$ must be represented based on a suitable convex multi-variable combination of the following arguments, namely

$$
\hat{W}\left(\boldsymbol{F}, \boldsymbol{H}, J, \boldsymbol{D}_{0}, \boldsymbol{d}\right)=w\left(I I_{\hat{\boldsymbol{F}}},\left(I I_{\hat{\boldsymbol{H}}}\right)^{3 / 2}, 1, I I_{\boldsymbol{D}_{0}}, I I_{\hat{d}}\right) .
$$

As pointed out in Reference [1], not every possible combination of the convex multi-variable invariants in (43), namely $I I_{\hat{\boldsymbol{F}}},\left(I I_{\hat{\boldsymbol{H}}}\right)^{3 / 2}, I I_{\boldsymbol{D}_{0}}$ and $I I_{\hat{\boldsymbol{d}}}$ will result in a convex multi-variable energy functional according to (6).

Following the work of Gil and Ortigosa [1] in the context of compressible convex multi-variable electromechanics, two regularisation techniques based on the convexification of a priori non convex multi-variable isochoric invariants will be presented in this Section.

\subsection{Stabilisation example 1}

In this section, the following non-convex multi-variable isochoric invariants will be considered

$$
W_{1}=\left(I I_{\hat{\boldsymbol{F}}}\right)^{m_{1}} I I_{\boldsymbol{D}_{\mathbf{0}}} ; \quad W_{2}=\left(I I_{\hat{\boldsymbol{H}}}\right)^{3 m_{2} / 2} I I_{\boldsymbol{D}_{\mathbf{0}}},
$$

where $m_{1} \geq 1$ and $m_{2} \geq 1$. Following the ideas in Reference [1], it is possible to show that even the one-dimensional representations of the invariants $W_{1}$ and $W_{2}$ in above equation (44) do not satisfy the required convexity conditions to guarantee material stability for the entire range of stretches $\lambda$ and electric displacements $D_{0}$. A suitable convex multi-variable isochoric invariant combination is

$$
\hat{W}\left(\boldsymbol{F}, \boldsymbol{H}, J, \boldsymbol{D}_{0}, \boldsymbol{d}\right)=\alpha \underbrace{\left(\left(I I_{\hat{\boldsymbol{F}}}\right)^{m_{1}}+\gamma^{2} I I_{\boldsymbol{D}_{\mathbf{0}}}\right)^{2}}_{W_{\hat{\boldsymbol{F}} \boldsymbol{D}_{0}}\left(\boldsymbol{F}, J, \boldsymbol{D}_{0}\right)}+\beta \underbrace{\left(\left(I I_{\hat{\boldsymbol{H}}}\right)^{3 m_{2} / 2}+\gamma^{2} I I_{\boldsymbol{D}_{\mathbf{0}}}\right)^{2}}_{W_{\hat{\boldsymbol{H}} \boldsymbol{D}_{0}}\left(\boldsymbol{H}, J, \boldsymbol{D}_{0}\right)}+f\left(\hat{\boldsymbol{F}}, \hat{\boldsymbol{H}}, \boldsymbol{D}_{0}, \hat{\boldsymbol{d}}\right),
$$

with $\alpha, \beta$ and $\gamma^{7}$ positive material parameters and $f\left(\hat{\boldsymbol{F}}, \hat{\boldsymbol{H}}, \boldsymbol{D}_{0}, \hat{\boldsymbol{d}}\right)$, a convex function of the elements of the extended set $\mathcal{V}$. Appropriate values for $\alpha, \beta$

\footnotetext{
${ }^{7}$ The material parameter $\gamma$ is employed for re-scaling purposes.
} 
and $\gamma$ and suitable functions $f$ must be such that, at the initial configuration, the stress vanishes, the Hessian operator (22) is positive definite and material characterisation can be carried out against available data. Since $f$ is convex multi-variable and $\alpha$ and $\beta$ are positive material parameters, sufficient conditions to ensure the multi-variable convexity of the functional (45) are the convexity of the energy contributions $W_{\hat{\boldsymbol{F}} \boldsymbol{D}_{0}}$ with respect to its arguments $\left\{\boldsymbol{F}, J, \boldsymbol{D}_{0}\right\}$ and $W_{\hat{\boldsymbol{H}}, \boldsymbol{D}_{0}}$ with respect to its arguments $\left\{\boldsymbol{H}, J, \boldsymbol{D}_{0}\right\}$ (refer to equation (45)). Proof of convexity of $W_{\hat{\boldsymbol{F}} \boldsymbol{D}_{0}}$ and $W_{\hat{\boldsymbol{H}}, \boldsymbol{D}_{0}}$ with respect to $\left\{\boldsymbol{F}, J, \boldsymbol{D}_{0}\right\}$ and $\left\{\boldsymbol{H}, J, \boldsymbol{D}_{0}\right\}$ can be found in Appendix C.1.

\subsection{Stabilisation example 2}

The stabilisation of the non-convex multi-variable isochoric invariant $\hat{\boldsymbol{h}}=$ $\hat{\boldsymbol{H}} \boldsymbol{D}_{0}$ inherits the same convex restrictions as the isochoric component of the co-factor $\hat{\boldsymbol{H}}$ (see Reference [40] in the context of pure elasticity and the second term on the right hand side of equation (15)). Hence, although it might be tempting to propose the following invariant regularisation

$$
\hat{W}_{\hat{\boldsymbol{H}} \boldsymbol{D}_{0}}=I I_{\hat{\boldsymbol{H}}}^{2}+\gamma^{2} I I_{\hat{\boldsymbol{h}}}+\gamma^{4} I I_{\boldsymbol{D}_{0}}^{2},
$$

the resulting invariant $\hat{W}_{\hat{\boldsymbol{H}} \boldsymbol{D}_{0}}$ is not convex multi-variable. Alternatively, a suitable definition of the invariant $\hat{W}_{\hat{\boldsymbol{H}} \boldsymbol{D}_{0}}$ complying with the multi-variable convexity condition in equation (6) is proposed as

$$
\hat{W}\left(\boldsymbol{F}, \boldsymbol{H}, J, \boldsymbol{D}_{0}, \boldsymbol{d}\right)=\alpha \underbrace{\left[I I_{\hat{\boldsymbol{H}}}^{3}+m \gamma^{2} I I_{\hat{\boldsymbol{h}}}^{3 / 2}+\gamma^{4} I I_{\boldsymbol{D}_{\mathbf{0}}}^{3}\right]}_{\hat{W}_{\hat{\boldsymbol{H}} \boldsymbol{D}_{0}}\left(\boldsymbol{H}, J, \boldsymbol{D}_{0}\right)}+f\left(\hat{\boldsymbol{F}}, \hat{\boldsymbol{H}}, \boldsymbol{D}_{0}, \hat{\boldsymbol{d}}\right),
$$

with $m \in(0,0.053)^{8}$ and with $\alpha, \beta$ and $\gamma^{9}$ positive material parameters and $f$ a convex multi-variable function. A sufficient condition to ensure the multi-variable convexity of the functional (47) is the convexity of the energy contribution $\hat{W}_{\hat{\boldsymbol{H}} \boldsymbol{D}_{0}}$ with respect to its arguments $\left\{\boldsymbol{H}, J, \boldsymbol{D}_{0}\right\}$ (refer to equation (47)). Proof of convexity of $\hat{W}_{\hat{H} \boldsymbol{D}_{0}}$ with respect to $\left\{\boldsymbol{H}, J, \boldsymbol{D}_{0}\right\}$ can be found in Appendix C.2.

\footnotetext{
${ }^{8}$ Appendix C.2 shows that a value of $m$ within the interval $m \in(0,0.053)$ satisfies the multi-variable convexity of the resulting invariant.

${ }^{9}$ The material parameter $\gamma$ is employed for re-scaling purposes.
} 


\section{Finite Element computational implementation}

This Section presents the finite element implementation of the variational principles $\Pi_{\Phi_{N I C}}(35), \Pi_{\Phi_{I}}(37), \Pi_{W_{N I C}}(40)$ and $\Pi_{W_{I}}$ (41), presented in Section 3.

\subsection{Finite Element implementation of standard displacement and electric} potential based formulation. Stabilised linear tetrahedron finite element

The finite element implementation of the variational principle $\prod_{\Phi_{N I C}}(35)$ will be discussed in this Section. The same results would be obtained for the variational principle $\Pi_{\Phi_{I}}$ (37) whereby the complementary energy needs to be particularised to $\Gamma(p)=-p$. In this case, we consider a discretisation where geometry, electric potential and pressure fields are interpolated using linear tetrahedral elements in terms of the same standard shape functions $N_{a}$ as

$$
\boldsymbol{x}=\sum_{a=1}^{4} \boldsymbol{x}_{a} N_{a} ; \quad \varphi=\sum_{a=1}^{4} \varphi_{a} N_{a} ; \quad p=\sum_{a=1}^{4} p_{a} N_{a} .
$$

Unfortunately, the resulting choice of interpolation spaces does not satisfy the LBB condition and leads to unstable solutions [67]. The classical solution to this problem is to introduce Petrov-Galerkin stabilisation [53, 60, 64, 91]. Following the work by Bonet et al. [46] in the context of polyconvex elasticity, such stabilisation entails a redefinition of the virtual velocity and pressure fields as

$$
\delta \boldsymbol{u}^{s t}=\delta \boldsymbol{u}-\tau_{\boldsymbol{v}}\left(\boldsymbol{H}_{\boldsymbol{x}} \boldsymbol{\nabla}_{0} \delta p\right) ; \quad \delta p^{s t}=\delta p-\tau_{p}\left(\boldsymbol{H}_{\boldsymbol{x}}: \boldsymbol{\nabla}_{0} \delta \boldsymbol{u}\right) .
$$

The stabilisation parameter $\tau_{p}$ has the same units as an elastic constant and $\tau_{\boldsymbol{v}}$ is defined following [91], namely $\tau_{\boldsymbol{v}}=\frac{\alpha h^{2}}{2 \mu}$, where $\mu$ is an elastic constant, $h$ is the mesh size and $\alpha$, a non-dimensional stabilisation parameter, typically ranging from 0 to 0.1 .

The discrete stationary conditions of the variational principle $\prod_{\Phi_{N I C}}(35)$ with respect to virtual changes in the geometry, electric potential and pressure (i.e. equilibrium, Gauss law and incompressibility constraint) are

$$
D \Pi_{\Phi_{N I C}}^{s t}[\delta \boldsymbol{u}]=\sum_{a=1}^{4} \boldsymbol{R}_{\boldsymbol{x}}^{a} \cdot \delta \boldsymbol{u}^{a} ; \quad D \Pi_{\Phi_{N I C}}^{s t}[\delta \varphi]=\sum_{a=1}^{4} R_{\varphi}^{a} \delta \varphi^{a} ; \quad D \Pi_{\Phi_{N I C}}^{s t}[\delta p]=\sum_{a=1}^{4} R_{p}^{a} \delta p^{a},
$$


with the associated residuals $\boldsymbol{R}_{x}^{a}, R_{\varphi}^{a}$ and $R_{p}^{a}$ defined as

$$
\begin{aligned}
\boldsymbol{R}_{\boldsymbol{x}}^{a} & =\int_{V} \boldsymbol{P}_{N I C}^{s t} \boldsymbol{\nabla}_{0} N_{a} d V-\int_{V} \boldsymbol{f}_{0} N_{a} d A-\int_{\partial_{t} V} \boldsymbol{t}_{0} N_{a} d V \\
R_{\varphi}^{a} & =\int_{V} \boldsymbol{D}_{0, \varphi} \cdot \boldsymbol{\nabla}_{0} N_{a} d V-\int_{V} \rho_{0} N_{a} d V-\int_{\partial_{\omega}} \omega_{0} d A \\
R_{p}^{a} & =\int_{V} N_{a}\left(J_{\boldsymbol{x}}+\frac{\partial \Gamma(p)}{\partial p}\right) d V-\int_{V} \tau_{\boldsymbol{v}} d V\left(\boldsymbol{H}_{\boldsymbol{x}} \boldsymbol{\nabla}_{0} N_{a}\right) \cdot\left(\boldsymbol{H}_{\boldsymbol{x}} \boldsymbol{\nabla}_{0} p\right) d V,
\end{aligned}
$$

where the stabilised first Piola-Kirchhoff stress tensors is defined as

$$
\boldsymbol{P}_{N I C}^{s t}=\boldsymbol{P}_{\boldsymbol{x}}+\tau_{p}\left(J_{\boldsymbol{x}}+\frac{\partial \Gamma(p)}{\partial p}\right) \boldsymbol{H}_{\boldsymbol{x}}
$$

with $\boldsymbol{P}_{\boldsymbol{x}}$ is additively decomposed in terms of its deviatoric and volumetric contributions as in equations $(29)_{a}$ and $(29)_{b}$, respectively.

The stiffness matrices arising from the variational principle $\prod_{\Phi_{N I C}}(35)$ emerge as

$$
D^{2} \Pi_{\Phi_{N I C}}[\delta \boldsymbol{u}, \delta \varphi ; \delta p ; \boldsymbol{u} ; \Delta \varphi ; \Delta p]=\left[\begin{array}{c}
\delta \mathbf{u} \\
\delta \boldsymbol{\phi} \\
\delta \mathbf{p}
\end{array}\right]^{T}\left[\begin{array}{ccc}
\boldsymbol{K}_{x \boldsymbol{x}} & \boldsymbol{K}_{\boldsymbol{x} \varphi} & \boldsymbol{K}_{\boldsymbol{x p}} \\
\boldsymbol{K}_{\varphi \boldsymbol{x}} & \boldsymbol{K}_{\varphi \varphi} & \mathbf{0} \\
\boldsymbol{K}_{p \boldsymbol{x}} & \mathbf{0} & \boldsymbol{K}_{p p}
\end{array}\right]\left[\begin{array}{c}
\mathbf{u} \\
\Delta \boldsymbol{\phi} \\
\Delta \mathbf{p}
\end{array}\right]
$$

The expressions for the non-zero blocks in the stiffness matrix defined in equation (53) are presented below. The diagonal component $\boldsymbol{K}_{\boldsymbol{x} \boldsymbol{x}}$ in above equation can be broken down into four components: a constitutive component associated to the isochoric stress contribution denoted as $\boldsymbol{K}_{\hat{\phi}}$, a volumetric component $\boldsymbol{K}_{p}$, an initial stress term $\boldsymbol{K}_{0}$ and a contribution from geometric derivative of the stabilisation term $\boldsymbol{K}_{\tau_{p}}$ as

$$
\boldsymbol{K}_{\boldsymbol{x} \boldsymbol{x}}=\left(\boldsymbol{K}_{\hat{\phi}}+\boldsymbol{K}_{p}+\boldsymbol{K}_{0}+\boldsymbol{K}_{\tau_{p}}\right) .
$$

The constitutive component of the isochoric contribution of the Helmhotz's energy functional $\hat{\Phi}$ gives

$$
\delta \boldsymbol{u}_{a} \cdot \boldsymbol{K}_{\hat{\Phi}}^{a b} \boldsymbol{u}_{b}=\int_{V}\left(\delta \boldsymbol{u}_{a} \otimes \nabla_{0} N_{a}\right): \hat{\mathcal{C}}^{*}:\left(\boldsymbol{u}_{b} \otimes \nabla_{0} N_{b}\right) d V,
$$


with $\hat{\mathcal{C}}^{*}$ defined in (32) and in (B.3) in terms of the components of the Hessian operator of the internal energy $\hat{e}\left(\boldsymbol{\nabla}_{0} \boldsymbol{x}, \boldsymbol{D}_{0}\right)$. Following References $[1,46]$, it is possible to obtain both contributions $\boldsymbol{K}_{0}$ and $\boldsymbol{K}_{\tau_{p}}$ as

$$
\left[\boldsymbol{K}_{p}^{a b}\right]_{i j}=\mathcal{E}_{i j k}\left[\boldsymbol{k}_{p}^{a b}\right]_{k} ; \quad\left[\boldsymbol{K}_{0}^{a b}\right]_{i j}=\mathcal{E}_{i j k}\left[\boldsymbol{k}_{0}^{a b}\right]_{k}
$$

with

$$
\begin{aligned}
\boldsymbol{k}_{p}^{a b} & =\int_{V} p \boldsymbol{F}_{x}\left(\boldsymbol{\nabla}_{0} N_{a} \times \boldsymbol{\nabla}_{0} N_{b}\right) d V \\
\boldsymbol{k}_{0}^{a b} & =\int_{V} \tau_{p}\left(J_{\boldsymbol{x}}+\frac{\partial \Gamma(p)}{\partial p}\right) \boldsymbol{F}_{\boldsymbol{x}}\left(\boldsymbol{\nabla}_{0} N_{a} \times \boldsymbol{\nabla}_{0} N_{b}\right) d V .
\end{aligned}
$$

Following Reference [46], it is possible to show that the last contribution of the stiffness $\boldsymbol{K}_{\boldsymbol{x} \boldsymbol{x}}$, i.e, $\boldsymbol{K}_{\tau_{p}}$ is defined as

$$
\boldsymbol{K}_{\tau_{p}}^{a b}=\int_{V} \tau_{p}\left(\boldsymbol{H}_{\boldsymbol{x}} \boldsymbol{\nabla}_{0} N_{a}\right) \otimes\left(\boldsymbol{H}_{\boldsymbol{x}} \boldsymbol{\nabla}_{0} N_{b}\right) d V
$$

The off-diagonal term $\boldsymbol{K}_{\boldsymbol{x} \varphi}$ (and similarly its symmetric counterpart $\boldsymbol{K}_{\varphi \boldsymbol{x}}$, $\boldsymbol{K}_{\varphi \boldsymbol{x}}=\boldsymbol{K}_{\boldsymbol{x} \varphi}$ ) yields

$$
\delta \boldsymbol{u}_{a} \cdot \boldsymbol{K}_{\boldsymbol{x} \varphi}^{a b} \Delta \varphi=\int_{V}\left(\boldsymbol{u}_{a} \otimes \nabla_{0} N_{a}\right): \mathcal{P}:\left(\varphi^{b} \nabla_{0} N^{b}\right) d V
$$

with $\mathcal{P}$ defined in equation (32). The diagonal stiffness $\boldsymbol{K}_{\varphi \varphi}$ is obtained as

$$
\boldsymbol{K}_{\varphi \varphi}=-\int_{V} \nabla_{0} N_{a} \cdot \varepsilon \nabla_{0} N_{b} d V
$$

with the dielectric tensor defined in equation (32). The off-diagonal stiffness contribution $\boldsymbol{K}_{x p}$ gives

$$
\boldsymbol{K}_{\boldsymbol{x} p}^{a b}=\int_{V} N_{b} \boldsymbol{H}_{\boldsymbol{x}} \boldsymbol{\nabla}_{0} N_{a} d V .
$$

Following Reference [46], the last off-diagonal stiffness contribution, namely $\boldsymbol{K}_{p \boldsymbol{x}}$ can be additively decomposed as

$$
\boldsymbol{K}_{p \boldsymbol{x}}=\boldsymbol{K}_{J}-\boldsymbol{K}_{\boldsymbol{H}},
$$


with

$$
\begin{aligned}
\boldsymbol{K}_{J}^{a b} & =\int_{V} N_{a} \boldsymbol{H}_{\boldsymbol{x}} \boldsymbol{\nabla}_{0} N_{b} d V \\
\boldsymbol{K}_{\boldsymbol{H}}^{a b} & =\int_{V} \tau_{\boldsymbol{v}}\left[\left(\boldsymbol{H}_{\boldsymbol{x}} \boldsymbol{\nabla}_{0} N_{a} \otimes \boldsymbol{\nabla}_{0} p\right) \times \boldsymbol{F}_{\boldsymbol{x}}+\left(\boldsymbol{H}_{\boldsymbol{x}} \boldsymbol{\nabla}_{0} p \otimes \boldsymbol{\nabla}_{0} N_{a}\right) \times \boldsymbol{F}_{\boldsymbol{x}}\right] \boldsymbol{\nabla}_{0} N_{b} d V .
\end{aligned}
$$

The last stiffness contribution, i.e, $\boldsymbol{K}_{p p}$ is finally obtained as

$$
\boldsymbol{K}_{p p}^{a b}=\int_{V} N_{a} N_{b} \frac{\partial^{2} \Gamma(p)}{\partial p^{2}} d V-\int_{V} \tau_{\boldsymbol{v}}\left(\boldsymbol{H}_{\boldsymbol{x}} \boldsymbol{\nabla}_{0} N_{a}\right)\left(\boldsymbol{H}_{\boldsymbol{x}} \boldsymbol{\nabla}_{0} N_{b}\right) d V .
$$

Notice that for the variational principle $\Pi_{\Phi_{I}}(37)$, the first term on the right hand side of above equation (64) would vanish as $\Gamma(p)=-p$.

\subsection{Finite element implementation twelve-field extended Hu-Washizu formu- lation}

The finite element implementation of the variational principle $\Pi_{W_{N I C}}(40)$ will be discussed in this Section. The same results would be obtained for the variational principle $\Pi_{W_{I}}$ (41), whereby the complementary energy needs to be particularised to $\Gamma(p)=-p$. For notational convenience, the following set $q$ of variables is introduced

$$
q=\left\{\boldsymbol{x}, \varphi, \mathcal{Y}, \Sigma_{\mathcal{Y}}, \boldsymbol{D}_{0}, p\right\}
$$

where the sets $\mathcal{Y}$ and $\Sigma_{\mathcal{Y}}$ are

$$
\mathcal{Y}=\{\boldsymbol{F}, \boldsymbol{H}, J, \boldsymbol{d}\} ; \quad \Sigma_{\mathcal{Y}}=\left\{\boldsymbol{\Sigma}_{\boldsymbol{F}}, \boldsymbol{\Sigma}_{\boldsymbol{H}}, \hat{\Sigma}_{J}, \boldsymbol{\Sigma}_{\boldsymbol{d}}\right\}
$$

Virtual and incremental variations of the elements in the sets $\mathcal{Y}$ and $\Sigma_{\mathcal{Y}}$ in above equation (66) are denoted as

$$
\begin{array}{rlrl}
\delta \mathcal{Y} & =\left\{\delta \boldsymbol{F}, \delta \boldsymbol{H}, \delta J, \delta \boldsymbol{\Sigma}_{\boldsymbol{D}_{0}}\right\} ; & \delta \Sigma_{\mathcal{Y}} & =\left\{\delta \boldsymbol{\Sigma}_{\boldsymbol{F}}, \delta \boldsymbol{\Sigma}_{\boldsymbol{H}}, \delta \hat{\Sigma}_{J}, \delta \boldsymbol{\Sigma}_{\boldsymbol{d}}\right\} \\
\Delta \mathcal{Y} & =\left\{\Delta \boldsymbol{F}, \Delta \boldsymbol{H}, \Delta J, \Delta \boldsymbol{\Sigma}_{\boldsymbol{d}}\right\} ; & \Delta \Sigma_{\mathcal{Y}}=\left\{\Delta \boldsymbol{\Sigma}_{\boldsymbol{F}}, \Delta \boldsymbol{\Sigma}_{\boldsymbol{H}}, \Delta \hat{\Sigma}_{J}, \Delta \boldsymbol{\Sigma}_{\boldsymbol{d}}\right\} .
\end{array}
$$

The implementation of the variational principle $\Pi_{W_{N I C}}(40)$ is based on a finite element partition (tesellation) of the domain $V$ (representing an electro active polymer in its initial configuration) into a set of elements. Inside each 
element, the problem variables $q$ (refer to equations (65) and (66)) can be interpolated in terms of a set of nodal shape functions as

$$
\begin{aligned}
& \boldsymbol{x}=\sum_{a=1}^{n_{\boldsymbol{x}}} \boldsymbol{x}_{a} N_{a}^{\boldsymbol{x}} ; \quad \varphi=\sum_{a=1}^{n_{\varphi}} \varphi_{a} N_{a}^{\varphi} ; \quad \mathcal{Y}=\sum_{a=1}^{n \mathcal{Y}} \mathcal{Y}_{a} N_{a}^{\mathcal{Y}} \\
& \Sigma_{\mathcal{Y}}=\sum_{a=1}^{n_{\Sigma_{\mathcal{Y}}}} \Sigma_{\mathcal{Y}_{a}} N_{a}^{\Sigma_{\mathcal{Y}}} ; \quad \boldsymbol{D}_{0}=\sum_{a=1}^{n_{\boldsymbol{D}_{0}}} \boldsymbol{D}_{0 a} N_{a}^{\boldsymbol{D}_{0}} ; \quad p=\sum_{a=1}^{n_{p}} p_{a} N_{a}^{p}
\end{aligned}
$$

where $a$ denotes the nodes used in the interpolation of the above variables and $n_{(\bullet)}$ denotes the number of nodes associated to the variable $(\bullet)$. The choice of interpolation spaces for the different variables considered will be described in Section 6.

The directional derivatives of the variational principle $\Pi_{W_{N I C}}(40)$ with respect to geometry $\boldsymbol{x}$, electric potential $\varphi$ and the additional unknown fields $\mathcal{Y}$, $\Sigma_{\mathcal{Y}}$ and $\boldsymbol{D}_{0}$ (and their discretisations), namely $D \Pi_{W_{N I C}}\left[\delta \boldsymbol{u}, \delta \varphi, \delta \mathcal{Y}, \delta \Sigma_{\mathcal{Y}}, \delta \boldsymbol{D}_{0}\right]$, have been presented in References $[1,2]$ in the context of compressible electromechanics. The only difference with respect to the formulation presented in References $[1,2]$ resides on the additive decomposition of the first PiolaKirchhoff stress tensor (refer to equation (13)), featuring in the directional derivative with respect to geometry.

The directional derivatives of the variational principle $\Pi_{W_{N I C}}(40)$ with respect to virtual variations of the pressure (not considered in References $[1,2])$ can be expressed as

$$
D \Pi_{W_{N I C}}[\delta p]=\int_{V} \delta p\left(J_{\boldsymbol{x}}+\frac{\partial \Gamma(p)}{\partial p}\right) d V
$$

The discretised expression for the above stationary condition (69) is

$$
D \Pi_{W_{N I C}}[\delta p]=\sum_{a=1}^{n_{p}} R_{p}^{a} \delta p^{a},
$$

where the residual $R_{p}^{a}$ is defined as

$$
R_{p}^{a}=\int_{V} N_{a}^{p}\left(J_{x}+\frac{\partial \Gamma(p)}{\partial p}\right) d V
$$


The stiffness matrices emerging after linearisation and discretisation of the variational principle $\Pi_{W_{N I C}}(40)$ emerge as

$$
D^{2} \Pi_{W_{N I C}}[\delta q ; \Delta q]=\left[\begin{array}{c}
\delta \mathbf{u} \\
\delta \boldsymbol{\phi} \\
\delta \mathcal{Y} \\
\delta \boldsymbol{\Sigma}_{\mathcal{Y}} \\
\delta \mathbf{D}_{0} \\
\delta \mathbf{p}
\end{array}\right]^{T}\left[\begin{array}{ccccccc}
\boldsymbol{K}_{\boldsymbol{x} \boldsymbol{x}} & \mathbf{0} & \mathbf{0} & \boldsymbol{K}_{\boldsymbol{x} \Sigma_{\mathcal{Y}}} & \boldsymbol{K}_{\boldsymbol{x} \boldsymbol{D}_{0}} & \boldsymbol{K}_{\boldsymbol{x} p} \\
\mathbf{0} & \mathbf{0} & \mathbf{0} & \mathbf{0} & \boldsymbol{K}_{\varphi \boldsymbol{D}_{0}} & \mathbf{0} \\
\mathbf{0} & \mathbf{0} & \boldsymbol{K}_{\mathcal{Y Y}} & \boldsymbol{K}_{\mathcal{Y} \Sigma_{\mathcal{Y}}} & \boldsymbol{K}_{\mathcal{Y} \boldsymbol{D}_{0}} & \mathbf{0} \\
\boldsymbol{K}_{\Sigma_{\mathcal{y}}} & \mathbf{0} & \boldsymbol{K}_{\Sigma_{\mathcal{Y}} \mathcal{Y}} & \mathbf{0} & \boldsymbol{K}_{\Sigma_{\mathcal{Y}} \boldsymbol{D}_{0}} & \mathbf{0} \\
\boldsymbol{K}_{\boldsymbol{D}_{0} \boldsymbol{x}} & \boldsymbol{K}_{\boldsymbol{D}_{0} \varphi} & \boldsymbol{K}_{\boldsymbol{D}_{0} \mathcal{Y}} & \boldsymbol{K}_{\boldsymbol{D}_{0} \Sigma_{\mathcal{Y}}} & \boldsymbol{K}_{\boldsymbol{D}_{0} \boldsymbol{D}_{0}} & \mathbf{0} \\
\boldsymbol{K}_{p \boldsymbol{x}} & \mathbf{0} & \mathbf{0} & \mathbf{0} & \mathbf{0} & \boldsymbol{K}_{p p}
\end{array}\right]\left[\begin{array}{c}
\mathbf{u} \\
\Delta \boldsymbol{\phi} \\
\Delta \mathcal{Y} \\
\Delta \boldsymbol{\Sigma}_{\mathcal{Y}} \\
\Delta \mathbf{D}_{0} \\
\Delta \mathbf{p}
\end{array}\right],
$$

where the expressions for the stiffness matrices within the upper left $5 \times 5$ block have been presented in Reference [2]. The off-diagonal contributions $\boldsymbol{K}_{\boldsymbol{x} p}$ and $\boldsymbol{K}_{p \boldsymbol{x}}$ in above (72) for the variational principle $\Pi_{W_{N I C}}$ (40) yields

$$
\boldsymbol{K}_{\boldsymbol{x} p}^{a b}=\int_{V} N_{b}^{p} \boldsymbol{H}_{\boldsymbol{x}} \boldsymbol{\nabla}_{0} N_{a}^{\boldsymbol{x}} d V ; \quad \boldsymbol{K}_{p \boldsymbol{x}}^{a b}=\left(\int_{V} N_{a}^{p} \boldsymbol{H}_{\boldsymbol{x}} \boldsymbol{\nabla}_{0} N_{b}^{\boldsymbol{x}} d V\right)^{T} .
$$

Finally, the $\boldsymbol{K}_{p p}$ contribution in above (72) can be expressed as

$$
\boldsymbol{K}_{p p}^{a b}=\int_{V} N_{a}^{p} N_{b}^{p} \frac{\partial^{2} \Gamma(p)}{\partial p^{2}} d V
$$

\section{Representative numerical examples}

The objective of this section is to compare the behaviour of the different mixed formulations presented in this paper. Regarding the mixed variational principles $\Pi_{W_{I}}$ (41) and $\Pi_{W_{N I C}}(40)$, two Finite Element discretisations have be used, named as $\mathbf{M W} \hat{\mathbf{W}}$ and $\mathbf{M} \hat{\mathbf{W F}}{ }^{+}$. For both formulations, a tetrahedral Finite Element has been used with piecewise linear (discontinuous) interpolation for the fields $\left\{\boldsymbol{F}, \boldsymbol{\Sigma}_{\boldsymbol{F}}, \boldsymbol{H}, \boldsymbol{\Sigma}_{\boldsymbol{H}}, \boldsymbol{D}_{0}, \boldsymbol{d}, \boldsymbol{\Sigma}_{\boldsymbol{d}}\right\}$. Moreover, a quadratic continuous interpolation of the electric potential field $\varphi$ and linear continuous interpolation of the pressure field has been considered in both $\mathbf{M W F}$ and $\mathbf{M} \hat{\mathbf{W F}}^{+}$discretisations. The differentiating element resides in the use of quadratic continuous interpolation for the geometry and constant interpolation of the fields $\left\{J, \hat{\Sigma}_{J}\right\}$ for the $\mathbf{M W F}$ discretisation. Notice that the constant interpolation of the fields $\left\{J, \hat{\Sigma}_{J}\right\}$ leads to a non-optimum order of convergence for the different fields involved in the formulation [2].

Alternatively, in order to preserve the optimum order of convergence, the $\mathbf{M} \hat{\mathbf{W F}}^{+}$considers a quadratic continuous interpolation of the geometry 
enriched with four cubic bubble functions added at the barycentre of each face of the tetrahedron and a quartic bubble function added at the barycentre of the tetrahedron itself. Additionally, a linear discontinuous interpolation of the fields $\left\{J, \hat{\Sigma}_{J}\right\}$ is considered in this discretisation. Notice that the resulting element is an extension of the classical $P 2^{+} P 1$ Crouzeix-Raviart element [92], typically used in $u-p$ formulations

Regarding the three-field mixed variational principles $\Pi_{\Phi_{N I C}}(35)$ and $\Pi_{\Phi_{I}}(37)$, three possible discretisations have been considered. The first one, denoted as P2P1, considers a tetrahedral Finite Element where a quadratic continuous interpolation of the geometry and the electric potential field is considered. Furthermore, a continuous linear interpolation of the pressure field is used. Notice that the resulting element is an extension of the classical P2P1 Taylor-Hood [92] element used in incompressible elasticity. The second possible discretisation has been denoted as B-bar. In this case, a hexahedral Finite Element where a trilinear continuous interpolation of the geometry and the electric potential field is used. The pressure field is constant within every element. The resulting element is an extension of the classical B-bar element used in incompressible elasticity [67]. Finally, the last discretisation used for the three-field formulation has been denoted as P1P1. In this case, a tetrahedral Finite Element where a linear continuous interpolation of the three unknown fields stabilised according to Section 5.1 has been employed.

Table 2 shows a helpful schematic representation of the different Finite Element spaces employed for the discretisation of the different mixed variational principles considered. 


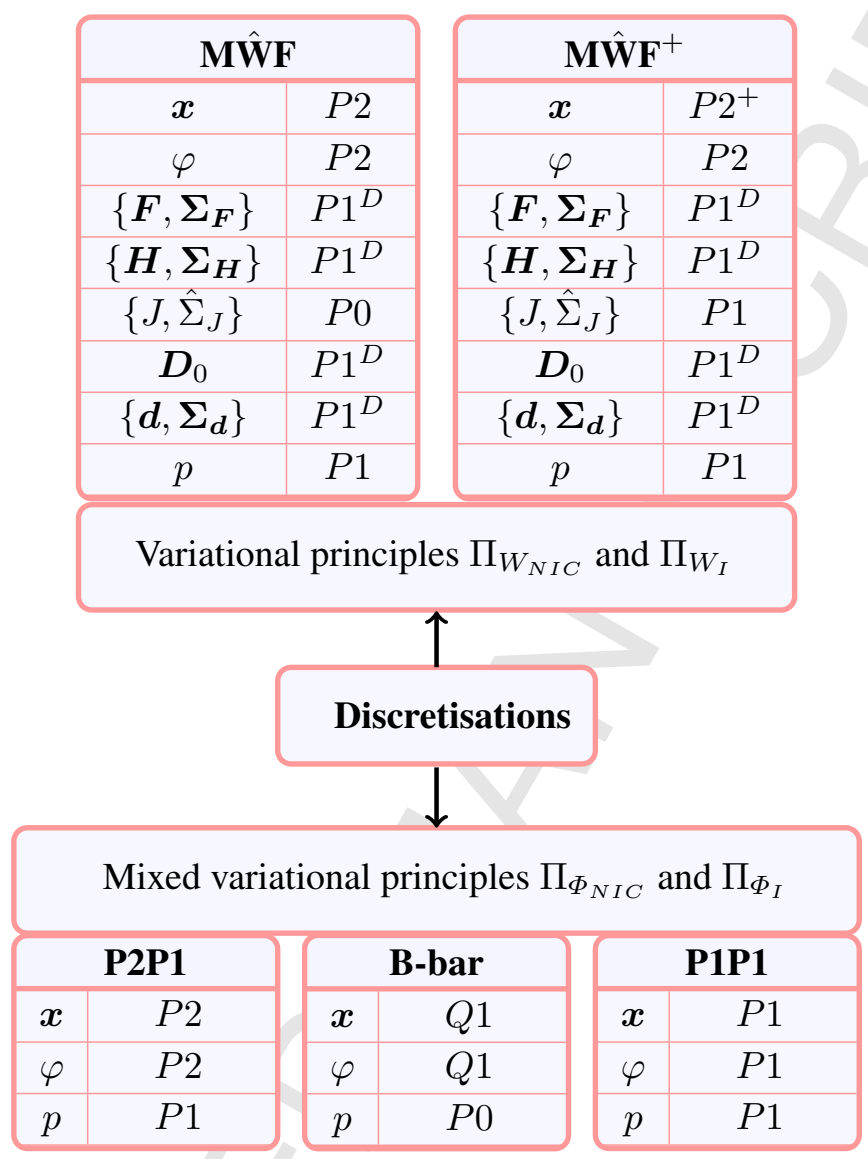

Figure 2: Different Finite Element spaces employed for the discretisation of the mixed variational principles $\Pi_{W_{N I C}}(40)$ and $\Pi_{I}$ (41) and for the threefield mixed variational principles $\Pi_{\Phi_{N I C}}$ (35) and $\Pi_{\Phi_{I}}$ (37). The superscript + denotes the use of stabilising bubble functions. The superscript $D$ denotes the use of element by element discontinuous interpolation spaces.

\subsection{Convergence of the proposed formulation}

The objective of this example is to demonstrate the $p$-order of accuracy of the different mixed formulations, as a function of the chosen finite element approximation spaces. The constitutive model considered is based on the 
following nearly incompressible convex multi-variable expansion

$$
W_{\text {simple }}=\mu_{1} J^{-2 / 3} I I_{\boldsymbol{F}}+\mu_{2} J^{-2} I I_{\boldsymbol{H}}^{3 / 2}+\frac{\kappa}{2}(J-1)^{2}+\frac{1}{2 \varepsilon_{1}} J^{-2 / 3} I I_{\boldsymbol{d}}+\frac{1}{2 \varepsilon_{2}} I I_{\boldsymbol{D}_{0}} .
$$

Notice that the material parameters in above constitutive model correspond to a shear modulus $\mu$, bulk modulus $\kappa_{0}$ and electric permittivity $\varepsilon$ in the reference configuration of,

$$
\mu=2 \mu_{1}+3 \sqrt{3} \mu_{2} ; \quad \kappa_{0}=\kappa ; \quad \frac{1}{\varepsilon}=\frac{1}{\varepsilon_{1}}+\frac{1}{\varepsilon_{2}} .
$$

The material parameters in (75) are presented in Table 1 , which correspond to a shear modulus of $\mu=4.5 P a$, a Poisson ratio of $\nu=0.49$ and an electric permittivity of $\varepsilon=1$.

\begin{tabular}{|c|c|c|c|c|}
\hline$\mu_{1}(P a)$ & $\mu_{2}(P a)$ & $\kappa(P a)$ & $\varepsilon_{1}\left(N / V^{2}\right)$ & $\varepsilon_{2}\left(N / V^{2}\right)$ \\
\hline 1 & $\frac{1}{2}$ & 228 & 2 & 2 \\
\hline
\end{tabular}

Table 1: Material properties for example 6.1.

In order to study the $p$-order of convergence of the new mixed Finite Element formulations, the analysis of an $a d-h o c$ test problem is carried out. This problem has been presented in Reference [2] in the context of compressible electro-elasticity. The problem is constructed so that smoothness of the solution is guaranteed. For that purpose, the following simple exact fields associated to the deformed or Eulerian configuration $\boldsymbol{x}$ and the electric potential $\varphi$ are considered

$$
\boldsymbol{x}^{\text {exact }}=\boldsymbol{X}+\left[\begin{array}{l}
A X_{1}^{3} \\
B X_{2}^{3} \\
C X_{3}^{3}
\end{array}\right] ; \quad \varphi^{\text {exact }}=\varphi_{0} X_{1}^{3},
$$

where the superscript in (77) is used to denote the exact solution with $A=$ $0.01, B=0.02, C=0.03$ and $\varphi_{0}=10^{-4}$. The deformation gradient tensor $\left(\boldsymbol{F}^{\text {exact }}=\boldsymbol{\nabla}_{0} \boldsymbol{x}^{\text {exact }}\right)$ and the Lagrangian electric field $\left(\boldsymbol{E}_{0}^{\text {exact }}=-\boldsymbol{\nabla}_{0} \varphi^{\text {exact }}\right)$ are obtained as

$$
\boldsymbol{F}^{\text {exact }}=\left[\begin{array}{ccc}
1+3 A X_{1}^{2} & 0 & 0 \\
0 & 1+3 B X_{2}^{2} & 0 \\
0 & 0 & 1+3 C X_{3}^{2}
\end{array}\right] ; \quad \boldsymbol{E}_{0}^{\text {exact }}=-\left[\begin{array}{c}
3 \varphi_{0} X_{1}^{2} \\
0 \\
0
\end{array}\right] .
$$


The remaining variables defining multi-variable convexity, namely $\left\{\boldsymbol{H}, J, \boldsymbol{D}_{0}, \boldsymbol{d}\right\}$ need to be obtained for the smooth displacement and electric potential fields in equation (77). Particularisation of equation (1) for the smooth fields in (77) enables $\boldsymbol{H}^{\text {exact }}$ and $J^{\text {exact }}$ to be obtained as

$$
\boldsymbol{H}^{\text {exact }}=\frac{1}{2} \boldsymbol{F}^{\text {exact }} \times \boldsymbol{F}^{\text {exact }} ; \quad J^{\text {exact }}=\frac{1}{3} \boldsymbol{H}^{\text {exact }}: \boldsymbol{F}^{\text {exact }} .
$$

Particularisation of equation $(12)_{b}$ to the constitutive model in equation (75) leads to

$$
\frac{1}{\varepsilon_{2}} \boldsymbol{D}_{0}^{\text {exact }}+\frac{1}{\varepsilon_{1}}\left(\boldsymbol{F}^{\text {exact }}\right)^{T} \boldsymbol{d}^{\text {exact }}=\boldsymbol{E}_{0}^{\text {exact }} .
$$

Making use of the relationship $\boldsymbol{d}^{\text {exact }}=\boldsymbol{F}^{\text {exact }} \boldsymbol{D}_{0}^{\text {exact }}$ in (80) results in the final expression of the Lagrangian electric displacement field $\boldsymbol{D}_{0}^{\text {exact }}$ as

$$
\left(\frac{1}{\varepsilon_{2}} \boldsymbol{I}+\frac{1}{\varepsilon_{1}} \boldsymbol{C}^{\text {exact }}\right) \boldsymbol{D}_{0}^{\text {exact }}=\boldsymbol{E}_{0}^{\text {exact }} \Rightarrow \boldsymbol{D}_{0}^{\text {exact }}=\left(\frac{1}{\varepsilon_{2}} \boldsymbol{I}+\frac{1}{\varepsilon_{1}} \boldsymbol{C}^{\text {exact }}\right)^{-1} \boldsymbol{E}_{0}^{\text {exact }} .
$$

where $\boldsymbol{C}^{\text {exact }}$ is the right Cauchy-Green tensor. Once all the elements of the set $\mathcal{V}^{\text {exact }}$ have been determined, it is possible to obtain the set of exact work conjugates $\Sigma_{\mathcal{V}}^{\text {exact }}$ via equation (12). Moreover, the hydrostatic pressure associated to the constitutive model in equation (75) for the smooth displacement field in equation (77) is obtained as

$$
p^{\text {exact }}=\kappa\left(J^{\text {exact }}-1\right) .
$$

The associated volumetric force and electric charge in mechanical and electrical equilibrium with the exact first Piola Kirchhoff stress tensor $\boldsymbol{P}^{\text {exact }}$ and exact Lagrangian electric displacement field $\boldsymbol{D}_{0}^{\text {exact }}$ are determined from equations (5) and (3a), respectively as

$$
\boldsymbol{f}_{0}\left(\boldsymbol{x}^{\text {exact }}, \varphi^{\text {exact }}\right)=-\operatorname{DIV} \boldsymbol{P}^{\text {exact }} ; \quad \rho_{0}\left(\boldsymbol{x}^{\text {exact }}, \varphi^{\text {exact }}\right)=\operatorname{DIV} \boldsymbol{D}_{0}^{\text {exact }},
$$

where $\boldsymbol{D}_{0}^{\text {exact }}$ has been obtained in (81) and where $\boldsymbol{P}^{\text {exact }}$ can be obtained after application of equation $(12)_{a}$. The analytical traction per unit undeformed area $\boldsymbol{t}_{0}$ and electric surface charge per unit undeformed area $\omega_{0}$ in the boundary of the dielectric elastomer associated to the smooth fields in equation (77) is obtained as

$$
\boldsymbol{t}_{0}^{\text {exact }}=\boldsymbol{P}^{\text {exact }} \boldsymbol{N} ; \quad \omega_{0}^{\text {exact }}=-\boldsymbol{D}_{0}^{\text {exact }} \cdot \boldsymbol{N},
$$


where $\boldsymbol{N}$ represents the normal vector in the boundary of the dielectric elastomer.

It is then that the rate of convergence of the different variables $\left\{\boldsymbol{x}, \varphi, \mathcal{V}, \Sigma_{\mathcal{V}}, p\right\}$ to their analytical counterparts, namely $\left\{\boldsymbol{x}^{\text {exact }}, \varphi^{\text {exact }}, \mathcal{V}^{\text {exact }}, \Sigma_{\mathcal{V}}^{\text {exact }}, p^{\text {exact }}\right\}$ can be studied. For that purpose, a computational domain corresponding to a cube such that $0 \leq X_{1} \leq 1,0 \leq X_{2} \leq 1$ and $0 \leq X_{3} \leq 1$ is initially discretised with $(2 \times 2 \times 2) \times 6$ tetrahedral elements and, subsequently, $h$-refinement is carried out generating a total of four discretisations. The convergence of these variables is studied for the $\mathbf{M} \hat{\mathbf{W F}}{ }^{+}$(using three discretisations), the B-bar (using three discretisations) and the stabilised P1P1 (using a total of four discretisations) Finite Elements.

Neumann boundary conditions are applied in the faces $X_{3}=0$ and $X_{3}=$ 1 , where the analytical traction and surface electric charge $\boldsymbol{t}_{0}^{\text {exact }}$ and $\omega_{0}^{\text {exact }}$ (refer to (84)) are applied. In the reminder faces of the cube, Dirichlet boundary conditions complying with the analytical fields $\boldsymbol{x}^{\text {exact }}$ and $\varphi^{\text {exact }}$ (refer to (84)) are applied.

The $L^{1}$ norm of the error for a particular component of the fields $\boldsymbol{x}, \boldsymbol{F}$, $\boldsymbol{H}, J, \boldsymbol{D}_{0}, \boldsymbol{d}, \boldsymbol{\Sigma}_{\boldsymbol{F}}, \boldsymbol{\Sigma}_{\boldsymbol{H}}, \hat{\Sigma}_{J}, \boldsymbol{\Sigma}_{\boldsymbol{D}_{0}}$ and $\boldsymbol{\Sigma}_{\boldsymbol{d}}$ is investigated for the $\mathbf{M} \hat{\mathbf{W}} \mathbf{F}$, the B-bar and the P1P1 elements.

Figure 3 shows the order of accuracy of the different unknown variables for the different mixed formulations. Figures 3(a), (c) and (e) display the convergence of the variables $\left\{\boldsymbol{x}, \boldsymbol{F}, \boldsymbol{H}, J, \boldsymbol{D}_{0}, \boldsymbol{d}\right\}$ for the $\mathbf{M} \hat{\mathbf{W F}} \mathbf{F}^{+}$, the $\mathbf{B}$-bar and the P1P1 elements whereas Figure 3(b), (d) and (f) display the convergence of the variables $\left\{\boldsymbol{\Sigma}_{\boldsymbol{F}}, \boldsymbol{\Sigma}_{\boldsymbol{H}}, \hat{\Sigma}_{J}, \boldsymbol{\Sigma}_{\boldsymbol{D}_{0}}, \boldsymbol{\Sigma}_{\boldsymbol{d}}, p\right\}$ for the those Finite Elements. The expected order of convergence is obtained for the different Finite Elements employed. It is worthwhile emphasising that the linear interpolation of both conjugate pairs $\left\{J, \Sigma_{J}\right\}$ and the addition of bubble functions for the interpolation of the geometry preserves the expected $p+1$ order of convergence for the $\mathbf{M} \hat{\mathbf{W F}}^{+}$element. Finally, as expected, the rate of convergence for the pressure is the same as that for the geometry and the electric potential in the stabilised $\mathbf{P} 1 \mathbf{P} 1$ formulation.

Finally, for completeness, the quadratic convergence of the Newton-Raphson algorithm is displayed in Figure 4.

\subsection{Bending actuator applications}

The objective of this section is to compare the solution obtained with the different formulations described above in scenarios where the applica- 


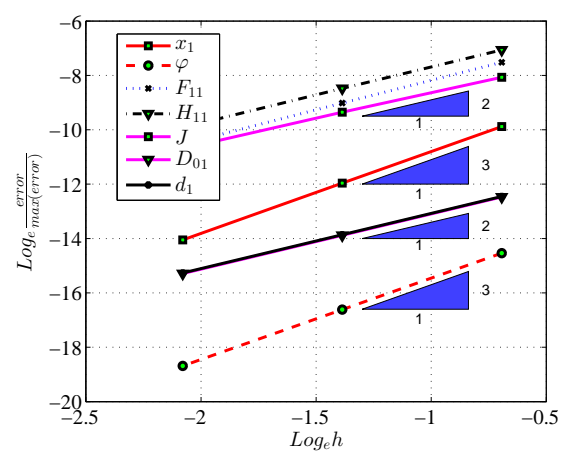

(a)

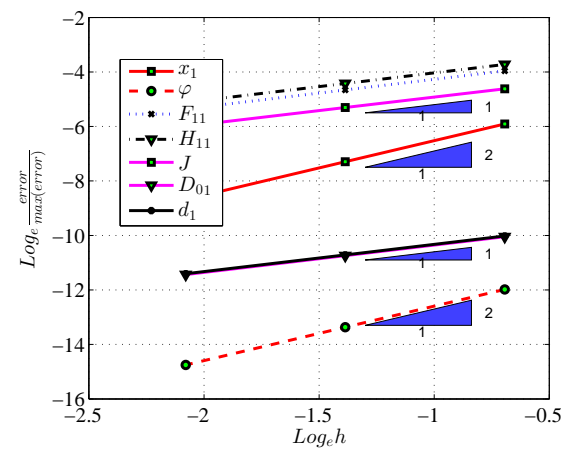

(c)

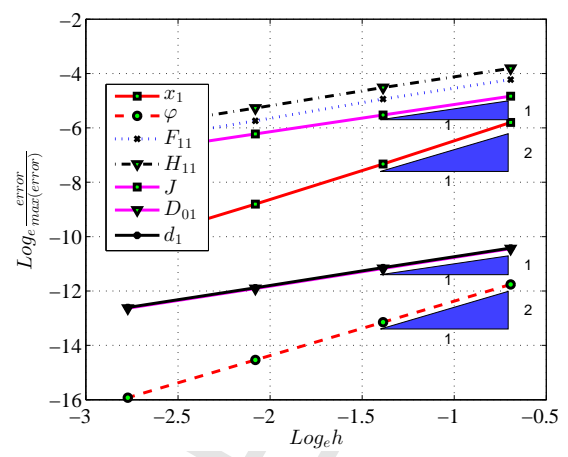

(e)

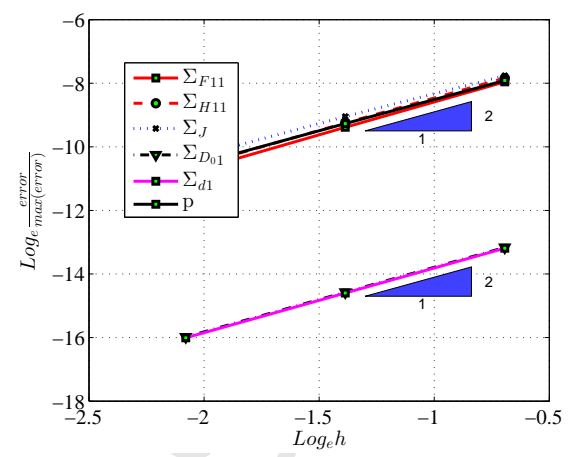

(b)

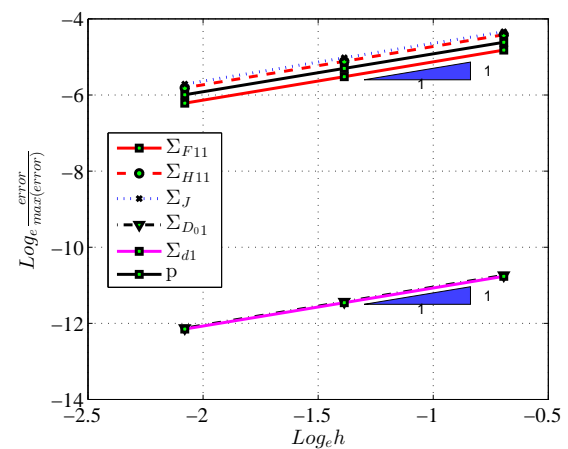

(d)

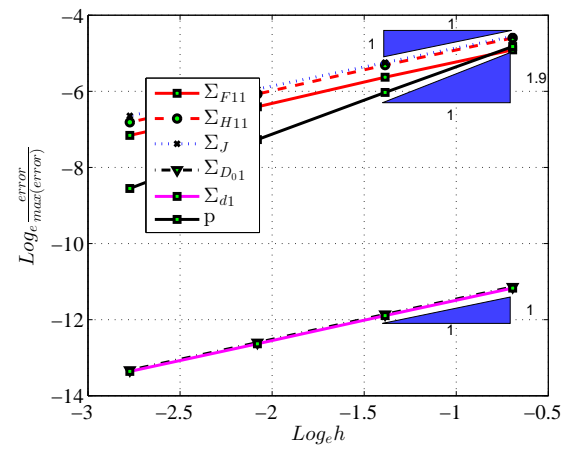

(f)

Figure 3: Convergence of the different formulations: order of accuracy of different strain, stress and electric magnitudes for the mixed formulations. Order of accuracy of the variables $\left\{\boldsymbol{x}, \varphi, \boldsymbol{F}, \boldsymbol{H}, J, \boldsymbol{D}_{0}, \boldsymbol{d}\right\}$ for (a) the $\mathbf{M} \hat{\mathbf{W}} \mathbf{F}^{+}$ formulation, (c) the B-bar formulation and (e), the P1P1 formulation. Order of accuracy of the variables $\left\{\boldsymbol{\Sigma}_{\boldsymbol{F}}, \boldsymbol{\Sigma}_{\boldsymbol{H}}, \hat{\Sigma}_{J}, \boldsymbol{\Sigma}_{\boldsymbol{D}_{0}}, \boldsymbol{\Sigma}_{\boldsymbol{d}}, p\right\}$ for (b) $\mathbf{M} \hat{\mathbf{W}} \mathbf{F}^{+}$ formulation, (d) the B-bar formulationt and (f), the P1P1 formulation. Constitutive model in equation (75). 


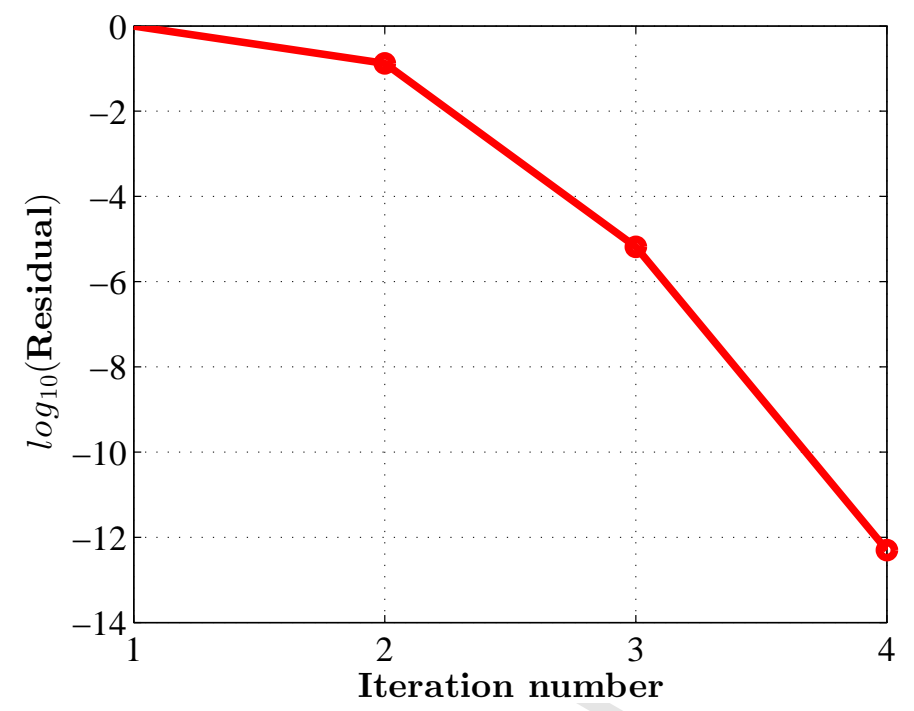

Figure 4: Quadratic convergence of the Newton-Raphson algorithm.

tion of an electric field on the electro active material leads to bending-type deformations.

\subsubsection{Bending actuator configuration 1}

The objective of this example is to compare the solution obtained with the different formulations described in the introduction of this section. In particular, the $\mathbf{M} \hat{\mathbf{W F}}$, the $\mathbf{P} \mathbf{2} \mathbf{P} \mathbf{1}$, the $\mathbf{B}-\mathbf{B a r}$ and the $\mathbf{P} \mathbf{1 P 1}$ Finite Elements will be compared.

This example considers the actuation device with geometry and boundary conditions described in Figure 5, where the application of an electric potential of $\varphi=0 \mathrm{~V}$ at $X_{3}=0.25 \mathrm{~m}$ and a surface electric charge per unit undeformed area $\omega_{0}$ at $X_{3}=0 \mathrm{~m}$ leads to a bending-type deformation around the axis $O X_{2}$ (refer to Figure 5). The deformation of the dielectric elastomer is constrained at both $X_{2}=0 \mathrm{~m}$ and $X_{2}=2 \mathrm{~m}$ in the direction parallel to the axis $O X_{2}$ and completely constrained at $X_{1}=0 \mathrm{~m}$. Finally, all the degrees of freedom for the displacement field are completely constrained at $X_{1}=0 \mathrm{~m}$.

The constitutive model used in this example is that of a simple incompressible ideal dielectric elastomer (with no electrostrictive effects), where the mechanical contribution of the electromechanical internal energy has been 


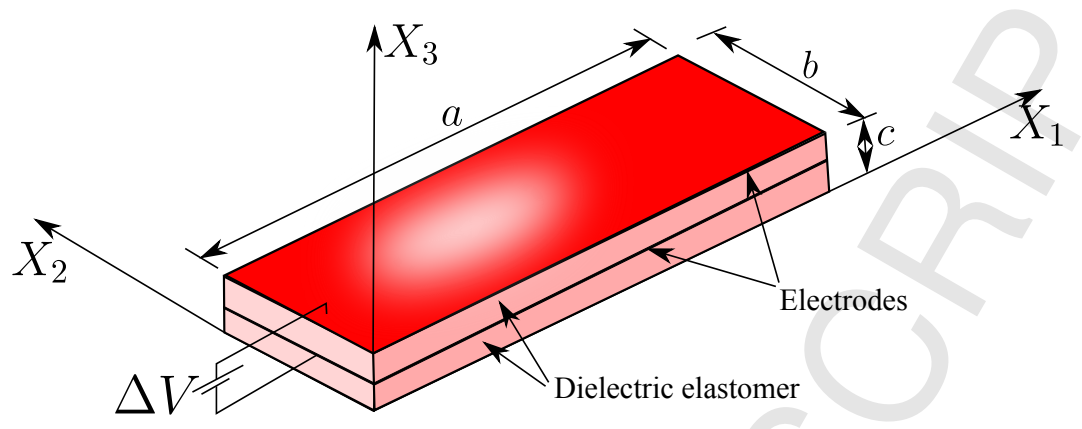

Figure 5: Bending actuator configuration 1: Electrical boundary conditions. $a=10 \mathrm{~m}, b=10 \mathrm{~m}$ and $c=0.5 \mathrm{~m}$.

chosen as that of an incompressible Mooney-Rivlin, shown in equation (15). The material parameters for this model are

$$
\eta=\frac{\mu}{4} ; \quad \gamma=\frac{\mu}{6 \sqrt{3}} ; \quad \varepsilon=4.68 \varepsilon_{0},
$$

with the shear modulus in the reference configuration defined as $\mu=10^{5} \mathrm{~Pa}$ and with a Poisson ratio of $\nu=0.5$. Hence a fully incompressible scenario is considered in this particular example.

Figure 6 presents a comparison for the $\mathbf{M W F}, \mathbf{P} \mathbf{2 P 1}$, B-bar and P1P1 Finite Elements for the Jacobian variable $J$, the hydrostatic pressure $p$ and the electric displacement field $\boldsymbol{D}_{0_{1}}$. All the results in this Figure have been obtained for the same value of the applied surface electric charge $\omega_{0}$. A value of $\alpha=0.1$ has been chosen for the stabilisation parameter (refer to equation (49)) in order to eliminate the presence of spurious pressure oscillations for the P1P1 element. Nevertheless, it is well known that this formulation suffers an additional shortcoming in bending dominated scenarios, namely bending locking $[57,60]$. However, $h$-refinement across the thickness of the domain alleviates this shortcoming. A post-processing nodal average of the pressure (pressure smoothing) has been applied for the B-bar element, yielding a qualitatively good agreement between all the formulations for the three variables considered. It is worthwhile emphasising that regarding the Jacobian variable $J$ (left column of above Figure 6), only in Figure 6(a) this variable $J$ is an unknown of the problem, as this result corresponds to the MWF element, which considers a constant interpolation of $J$ and its work conjugate $\Sigma_{J}$. 


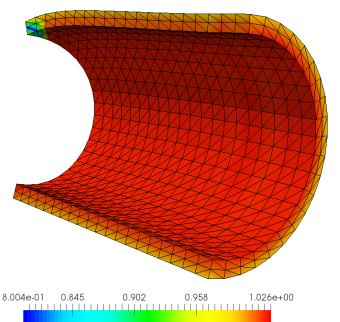

(a)

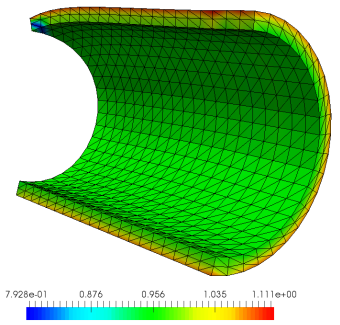

(d)

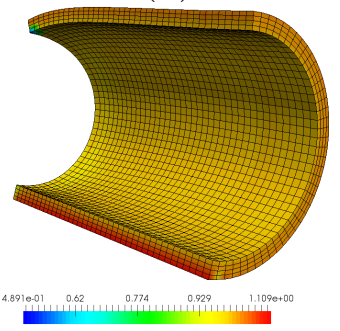

(g)

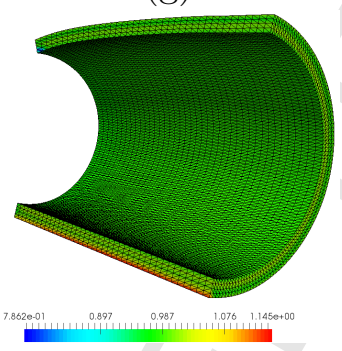

(j)

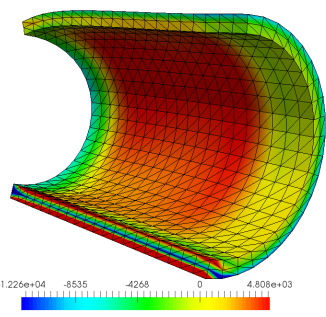

(b)

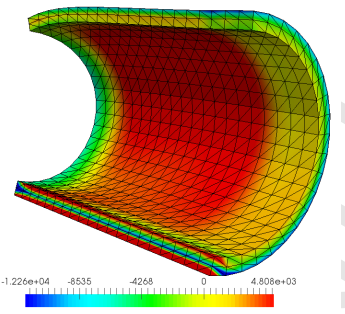

(e)

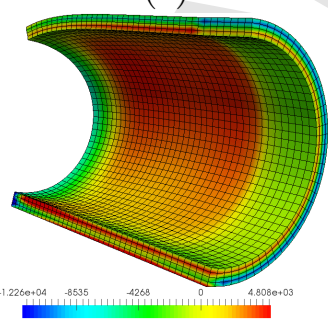

(h)

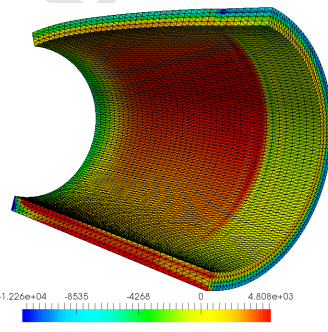

(k)

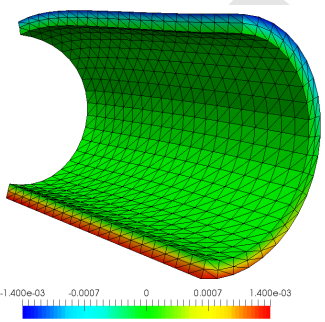

(c)

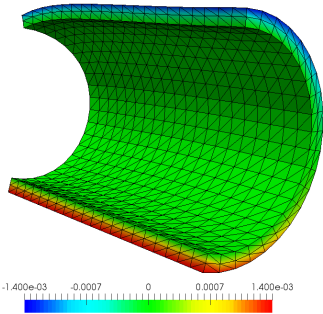

(f)

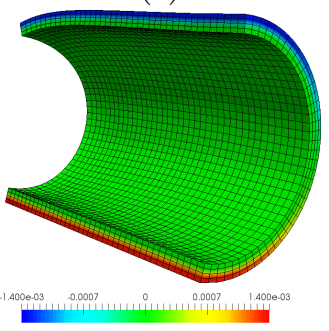

(i)

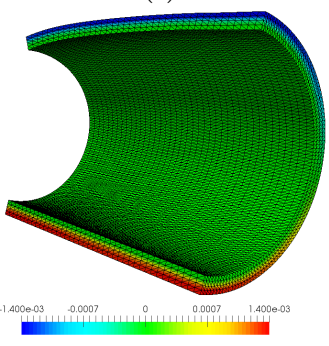

(l)

Figure 6: Bending actuator configuration 1. Contour plot of the Jacobian $J$ (left column), hydrostatic pressure $p$ (centre column) and the electric displacement field component $\boldsymbol{D}_{0_{1}}$ for (a), (b), (c) the MWWF element; (d), (e), (f) the P2P1 element; (g), (h), (i) the B-bar element and (j), (k), (l), the P1P1 element. Incompressible model in equation (15). Results obtained for a value of the applied surface electric charge of $\omega_{0}=1.5 \times 10^{-3} \mathrm{Q} / \mathrm{m}^{2}$ using a discretisation of $(20 \times 20 \times 2) \times 6$ elents for the $\mathbf{M W F}$ and $\mathbf{P} 2 \mathbf{P} 1$ Finite Elements; $40 \times 40 \times 4$ for the B-bar element and $(100 \times 40 \times 4) \times 6$ for the P1P1 element. 


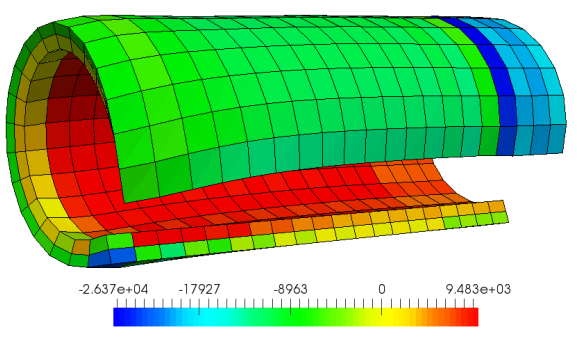

(a)

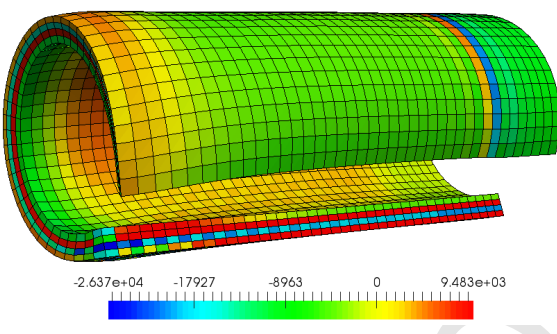

(c)

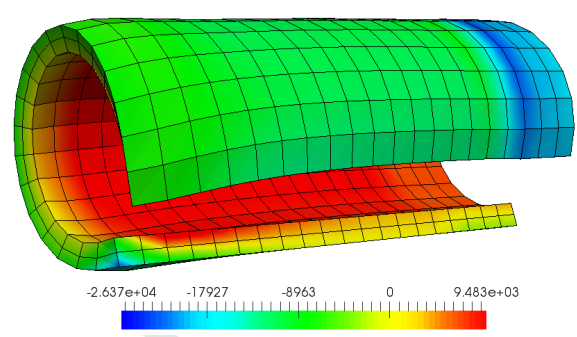

(b)

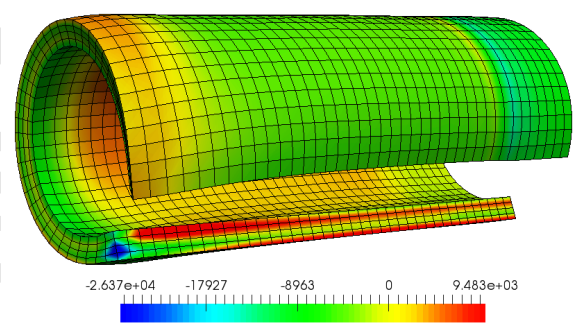

(d)

Figure 7: Bending actuator configuration 1. Comparison of results for the B-bar element: (a) without a posteriori pressure smoothing for $20 \times 20 \times 2$ elements; (b) with a posteriori pressure smoothing for $20 \times 20 \times 2$ elements; (c) without a posteriori pressure smoothing for $40 \times 40 \times 4$ elements; with a posteriori pressure smoothing for $40 \times 40 \times 4$ elements. Incompressible constitutive model in equation (15). Results obtained for a value of the applied surface electric charge of $\omega_{0}=2 \times 10^{-3} \mathrm{Q} / \mathrm{m}^{2}$. 


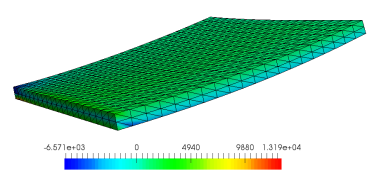

(a)

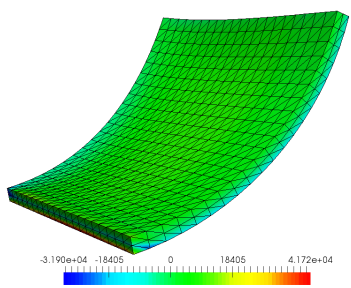

(d)

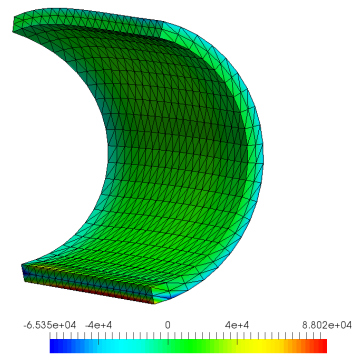

$(\mathrm{g})$

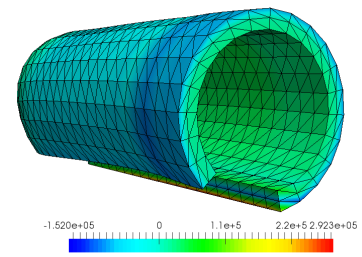

(j)

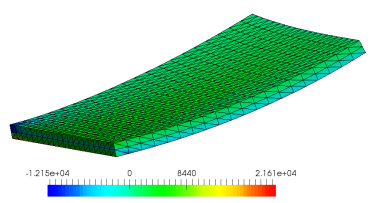

(b)

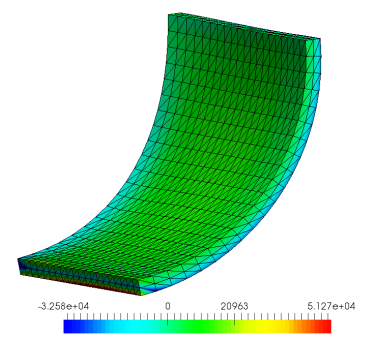

(e)

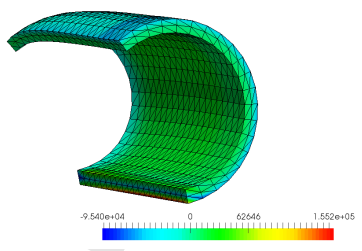

(h)

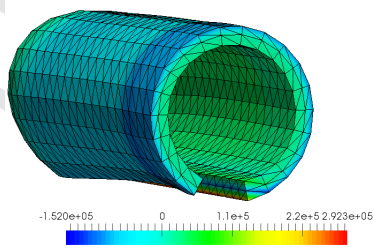

$(\mathrm{k})$

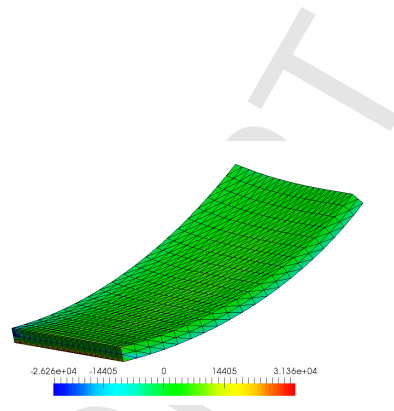

(c)

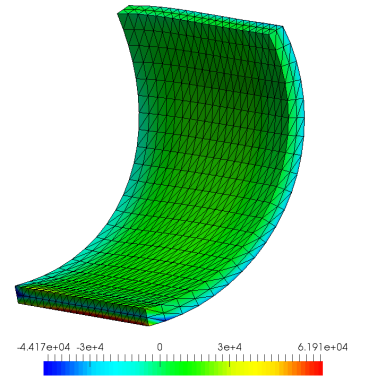

(f)

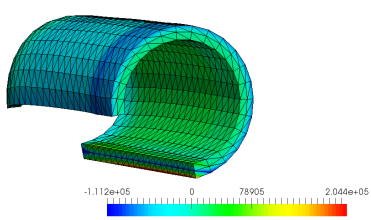

(i)

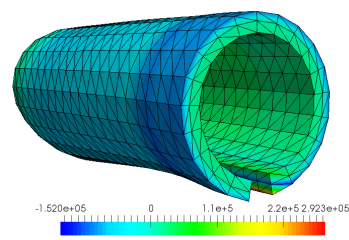

(l)

Figure 8: Bending actuator configuration 1. Contour plot of the pressure $p$ with the $\mathbf{M W F}$ element for an applied surface electric charge $\omega_{0}=\lambda \times(3 \times$ $10^{-3}$ ) $Q / m^{2}$, for a value of the accumulated factor $\lambda$ of: (a) $\lambda=0.107$, (b) $\lambda=0.146$, (c) $\lambda=0.218$, (d) $\lambda=0.272$, (e) $\lambda=0.317$, (f) $\lambda=0.359$, (g) $\lambda=0.437$, (h) $\lambda=0.513$, (i) $\lambda=0.593$, (j) $\lambda=0.636$, (k) $\lambda=0.659$ and (l) $\lambda=0.683$. Incompressible model in equation (15) using a discretisation of $(20 \times 20 \times 2) \times 6$ elements. 
Very interestingly, Figure 7 illustrates the presence of pressure modes associated with the B-bar element in the context of a coupled electromechanical problem. Figure 7(a) shows the contour plot of the pressure variable $p$ obtained with this formulation when pressure smoothing ${ }^{10}$ is not considered, hence, displaying a constant value of the pressure for every element of the computational domain. It can be observed that there some oscillations in the pressure field which could indicate the presence of a mode. However, 7(b) shows the contour plot of $p$ when pressure smoothing is applied at the post-processing level. Both Figures, namely 7(a) and 7(b) have been obtained for a coarse discretisation.

In order to corroborate the presence of the pressure mode, a fine mesh has been considered in both Figures $7(\mathrm{c})$ and $7(\mathrm{~d})$. Figure $7(\mathrm{a})$ shows the contour plot of the pressure variable $p$ obtained with this formulation when pressure smoothing is not considered. Clearly, the pressure field shows spurious oscillations which are now exacerbated in comparison to the coarser discretisation showed in Figure 7(a). Figure 7(d), clearly shows that pressure smoothing helps alleviating this detrimental feature associated to the B-bar element.

Finally, Figure 8 displays the contour plot of the pressure and the evolution of the deformation of the dielectric elastomer as the value of the applied surface electric charge $\omega_{0}$ increases, leading to the final extremely large deformation shown in Figure 8(l). These results have been obtained with the MŴF element.

\subsubsection{Bending actuator configuration 2}

In this section, the electrical boundary conditions, depicted in Figure 9, are different to those described in the previous example in Section 6.2.1, leading to a more complicated electrically deformation pattern. A fixed value of the electric potential of $\varphi=0 \mathrm{~V}$ is prescribed at $X_{3}=0.25 \mathrm{~m}$. Then, equal values of the applied surface electric charge per unit undeformed area $\omega_{0}$ are applied in the region $0 m \leq X_{1} \leq 5 m$ at $X_{3}=0 m$ and in the region $5 m \leq X_{1} \leq 10 m$ at $X_{3}=0.5 m$. The deformation of the dielectric elastomer is completely constrained at $X_{1}=0 \mathrm{~m}$ and the deformation in the direction parallel to the axis $O X_{2}$ is constrained at $X_{2}=0 \mathrm{~m}$.

\footnotetext{
${ }^{10}$ The pressure smoothing is carried out by averaging each of the constant pressure element contributions associated with a particular node of the Finite Element mesh.
} 
The constitutive model considered in this example corresponds to an incompressible electrostrictive dielectric elastomer, with an electromechanical internal energy defined as

$$
\begin{aligned}
\hat{W} & =\mu_{1} J^{-2 / 3} I I_{\boldsymbol{F}}+\mu_{2} J^{-2} I I_{\boldsymbol{H}}^{3 / 2}+\frac{J^{-2 / 3}}{2 \varepsilon_{1}} I I_{\boldsymbol{d}}+\frac{1}{2} I I_{\boldsymbol{D}_{0}} \\
& +\mu_{e} J^{-4 / 3}\left(I I_{\boldsymbol{F}}^{2}+\frac{2}{\mu_{e} \varepsilon_{e}} I I_{\boldsymbol{F}} I I_{\boldsymbol{d}}+\frac{1}{\mu_{e}^{2} \varepsilon_{e}^{2}} I I_{\boldsymbol{d}}^{2}\right) .
\end{aligned}
$$

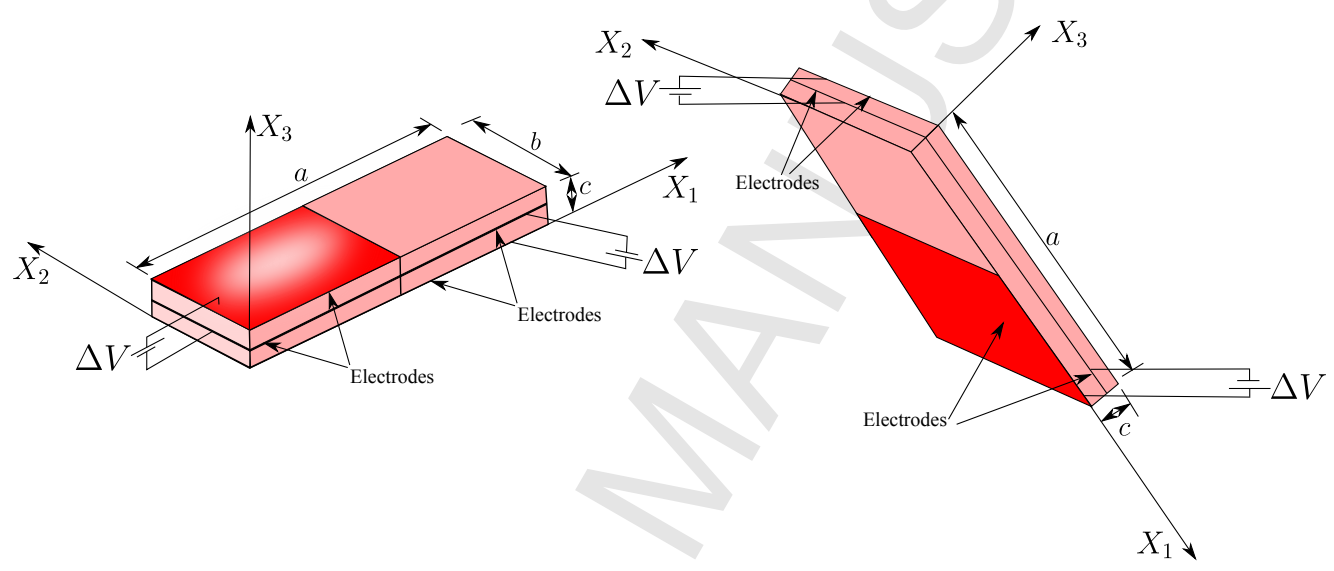

Figure 9: Bending actuator configuration 2: Electrical boundary conditions. $a=10 m, b=2 m$ and $c=0.5 m$.

Notice that the above constitutive model is convex multi-variable in the sense described in equation (6). The last invariant in above equation (86) accounts for electrostrictive and electric saturation effects (refer to References $[1,2])$. Notice that this invariant includes the non-convex multi-variable invariant $I I_{\boldsymbol{F}} I I_{\boldsymbol{d}}$, which has been stabilised via the convexification strategy described in Section 4.1.

The constitutive model described by the energy functional in (86) represents a generalisation of the electrostrictive convex multi-variable constitutive model proposed by Gil and Ortigosa in References $[1,2]$ to the context of nearly and fully incompressible electro-elasticity. In this model, the material parameters $\mu_{1}, \mu_{2}$ and $\mu_{e}$ are elastic parameters $\left(N / m^{2}\right)$ and both $\varepsilon_{1}$ and $\varepsilon_{e}$ are electric parameters with units $\left(N / V^{2}\right)$. Particularisation of the deviatoric elasticity tensor $\hat{\mathcal{C}}$ and the dielectric tensor $\boldsymbol{\theta}$ in equations (19), 
(A.2) and (A.9) to the reference configuration, namely $\boldsymbol{F}=\boldsymbol{H}=\boldsymbol{I}, J=1$ and $\boldsymbol{d}=\boldsymbol{D}_{0}=\mathbf{0}$, enables the material parameters in (86) to be related to the shear modulus and dielectric permittivity of the material in the reference configuration, namely $\mu$ and $\varepsilon$ as

$$
2 \mu_{1}+3 \sqrt{3} \mu_{2}+4 \mu_{e}=\mu ; \quad \frac{1}{\varepsilon_{1}}+\frac{1}{\varepsilon_{2}}+\frac{12}{\varepsilon_{e}}=\frac{1}{\varepsilon_{r} \varepsilon_{0}},
$$

with $\mu=10^{5} \mathrm{~Pa}$ and $\varepsilon_{r}=4.68$. Following Reference [1], the amount of electrostriction and electric saturation can be controlled via two auxiliar parameters, namely $f_{e}$ and $f_{s}$, respectively defined as

$$
\varepsilon_{1}=f_{e} \varepsilon ; \quad 12 \mu_{e}=f_{s} \mu .
$$

The value of the material parameters chosen for this particular example are shown in Table 2.

\begin{tabular}{|c|c|c|c|c|c|}
\hline$\mu_{1}(P a)$ & $\mu_{2}(P a)$ & $\varepsilon_{1}\left(N / V^{2}\right)$ & $\left(\varepsilon_{2}\right)^{-1}\left(V^{2} / N\right)$ & $f_{e}$ & $f_{s}$ \\
\hline $0.225 \mu$ & $0.5 \mu_{1}$ & $4.68 \varepsilon_{0}$ & 0 & 1.0515 & 0.0996 \\
\hline
\end{tabular}

Table 2: Material properties for example 6.2.2. Parameters $f_{e}$ and $f_{s}$ defined in equation (88), respectively.

Figure 10 presents a comparison for the $\mathbf{M W} \hat{\mathbf{W}}, \mathbf{P} \mathbf{2 P 1}$, B-bar and P1P1 Finite Elements for the Jacobian variable $J$, the hydrostatic pressure $p$ and the electric displacement field $\boldsymbol{D}_{0_{1}}$. All the results in this Figure have been obtained for the same value of the applied surface electric charge $\omega_{0}$. A value of $\alpha=0.1$ has been chosen for the stabilisation parameter (refer to equation (49)) for the P1-P1 formulation. A post-processing nodal average of the pressure (pressure smoothing) has been applied for the B-bar formulation, yielding a qualitatively good agreement between all the formulations for the three variables considered, except for the $\mathbf{P 1 P} 1$ element, strongly affected by bending locking. It is worthwhile emphasising that regarding the Jacobian variable $J$, it is the $\mathbf{M} \hat{\mathbf{W}} \mathbf{F}$ element whose contour plot distribution whose extreme values are closer to 1 . Notice that irrespectively of the element considered, the incompressibility condition is satisfied in a weak sense. Hence, it is not expected to obtain a distribution for the Jacobian variable exactly equal to one at each gauss node of the computational domain. 


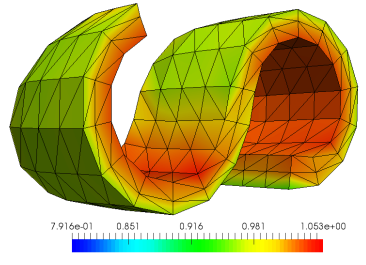

(a)

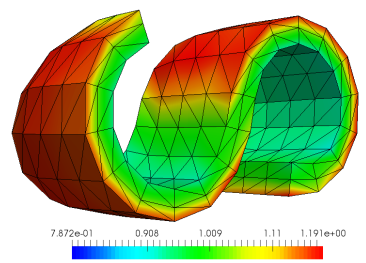

(d)

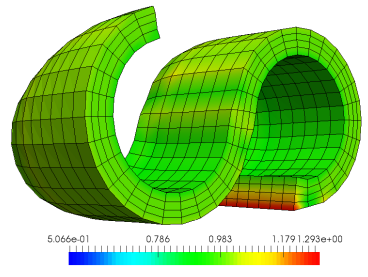

$(\mathrm{g})$

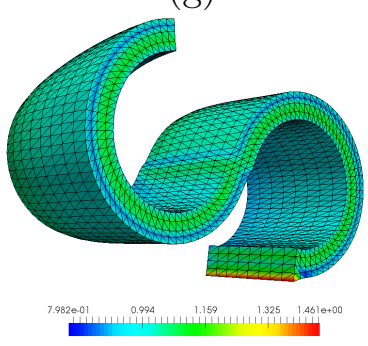

(j)

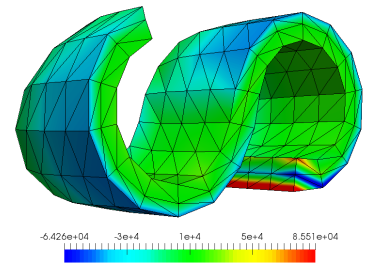

(b)

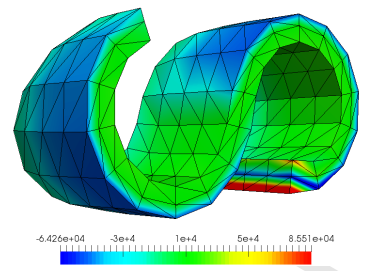

(e)

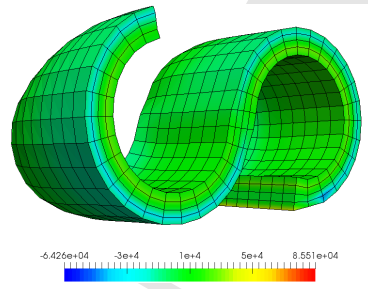

(h)

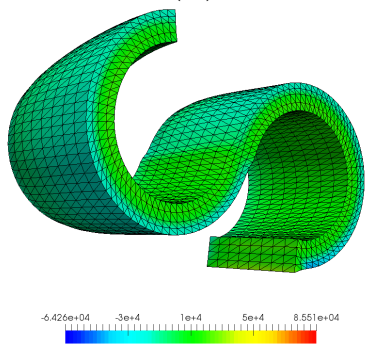

$(\mathrm{k})$

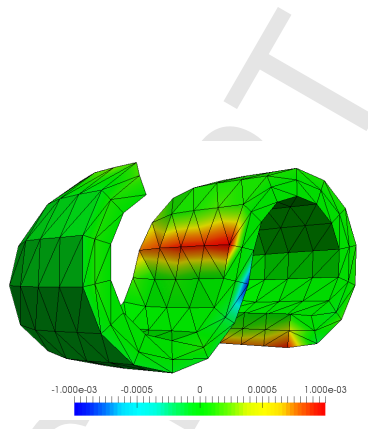

(c)

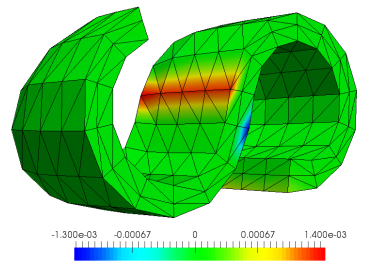

(f)

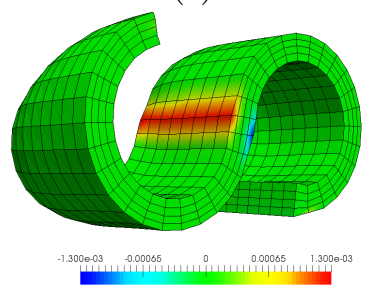

(i)

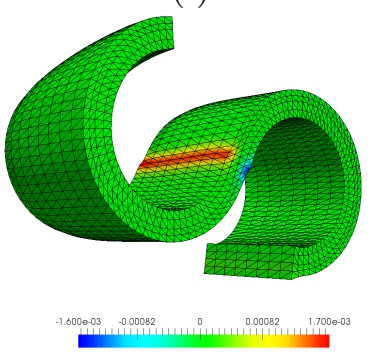

(1)

Figure 10: Bending actuator configuration 2. Contour plot of Jacobian variable $J$ (left column), hydrostatic pressure $p$ (center column) and the electric displacement field component $\boldsymbol{D}_{0_{1}}$ for (a), (b), (c) the $\mathbf{M} \hat{\mathbf{W F}}$ formulation; (d), (e), (f) the P2P1 element; (g), (h), (i) the B-bar formulation and (j), (k), (l), the P1P1 element. Incompressible constitutive model in equation (86). Results obtained for a value of the applied surface electric charge of $\omega_{0}=3 \times 10^{-3} \mathrm{Q} / \mathrm{m}^{2}$. Results for a discretisation of $(20 \times 4 \times 2) \times 6$ elements for the $\mathbf{M W F}$ and P2P1 elements; $40 \times 8 \times 4$ for the B-bar element and $(100 \times 8 \times 4) \times 6$ for the $\mathbf{P} \mathbf{1 P 1}$ element. 
An additional objective of this example is the study of the influence of the stabilisation parameter $\alpha\left(\tau_{\boldsymbol{v}}=\frac{\alpha h^{2}}{2 \mu}\right)$ in the stabilised P1P1 element. Different values of the stabilisation parameters $\alpha$ are considered in Figure 11 for this formulation. The results for the pressure are then compared to the more accurate $\mathbf{P} \mathbf{2 P} 1$ element for the same value of the applied surface electric charge $\omega_{0}$. It is clear that for low values of $\alpha$ (Figure 11(a)), the pressure contour shows spurious oscillations. For high values of $\alpha$ (Figure 11(e)), excessive dissipative results are obtained. For this particular example, it seems that the optimum value of the stabilisation parameter $\alpha$ which renders comparable results to those of the P2P1 element (Figure 11(f)) is $\alpha=0.05$.

Finally, Figure 12 displays the contour plot of the pressure and the evolution of the deformation of the dielectric elastomer as the value of the applied surface electric charge $\omega_{0}$ increases, leading to the final extremely large deformation shown in Figure 12(l). These results have been obtained with the B-bar element.

\subsection{Torsional actuator applications}

The objective of this example is to observe how the behaviour of an incompressible dielectric elastomer matrix can be modified when fibres are introduced in a specific direction characterised by the unit normal vector $\boldsymbol{N}$. This example is exactly the same as example 5.4 described in Reference [2], with the particularity that the material considered hereby is incompressible. The geometry and boundary conditions and the arrangement of the fibres (spherically parametrised) have been included in Figure 13 for the sake of completeness.

In this example, we consider the same distribution of fibres in both regions (unlike in Reference [2]) characterised by a preferred direction of $\boldsymbol{N}=\left[\begin{array}{lll}0 & \sqrt{2} / 2 & \sqrt{2} / 2\end{array}\right]^{T}(\psi=\pi / 4, \theta=\pi / 2)$. The material is incompressible. The constitutive model is based on an additive decomposition accounting for the matrix contribution and for the fibres. The matrix contribution has been defined according to the energy functional in equation (15) whereas the fibres contribution do not obey isochoric-volumetric split, in agreement with the physically motivated argument in Reference [93]. The convex multi-variable internal energy of the resulting constitutive model is defined as

$$
W=\hat{W}_{\text {matrix }}+W_{\text {fibre }}(\boldsymbol{F}, \boldsymbol{H}, J, \boldsymbol{N}),
$$




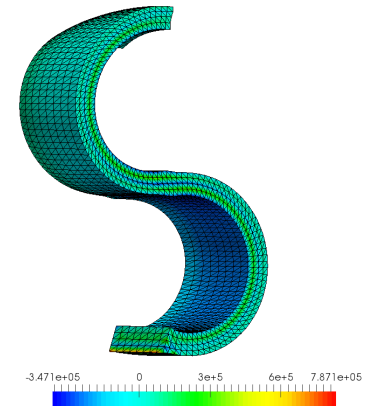

(a)

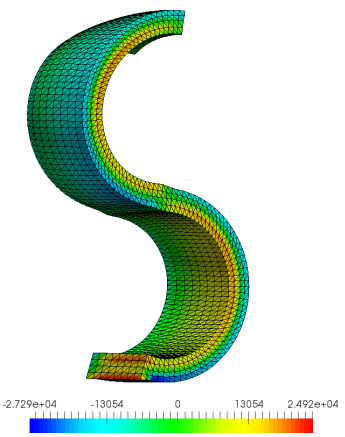

(d)

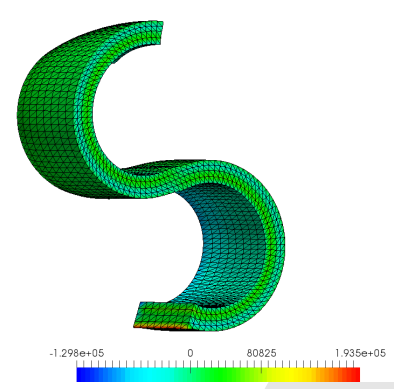

(b)

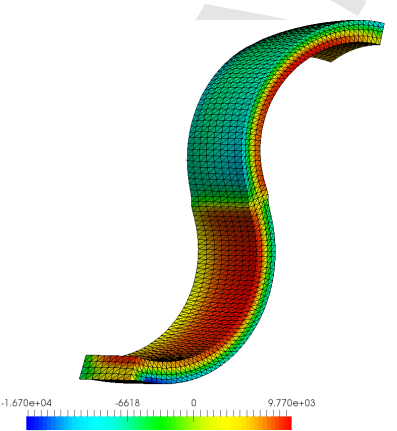

(e)

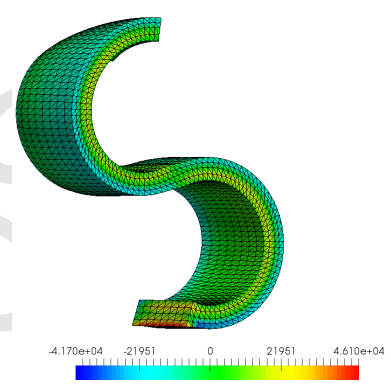

(c)

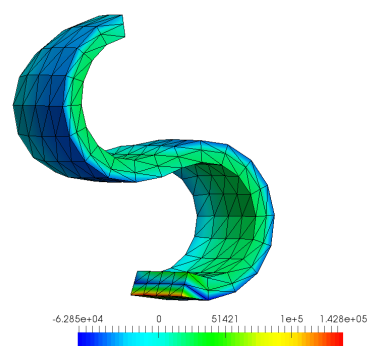

(f)

Figure 11: Bending actuator configuration 2. Contour plot of the hydrostatic pressure $p$ for the $\mathbf{P 1 P} \mathbf{1}$ element for a value of the stabilisation parameter $\alpha$ of: (a) $\alpha=0.0005$, (b) $\alpha=0.005$, (c) $\alpha=0.05$, (d) $\alpha=0.1$, (e) $\alpha=0.6$, (f) P2P1 element (for comparison purposes). Incompressible constitutive model in equation (86). Fixed value of the surface electric charge per unit undeformed area of $\omega_{0}=3 \times 10^{-2} \mathrm{Q} / \mathrm{m}^{2}$. 


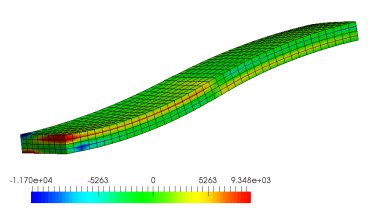

(a)

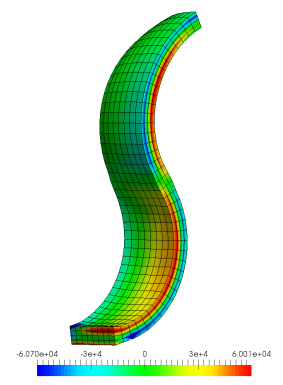

(d)

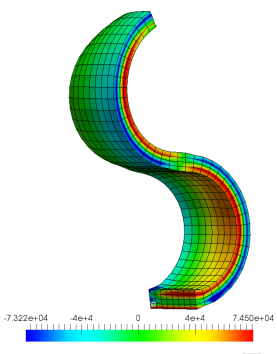

$(\mathrm{g})$

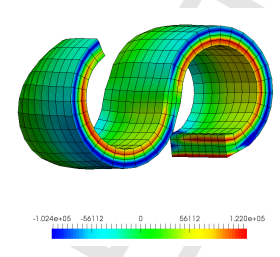

(j)

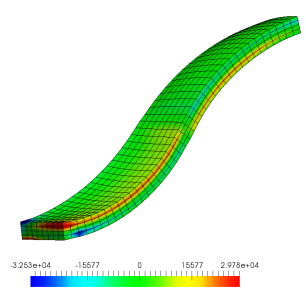

(b)

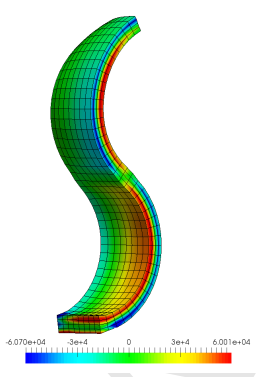

(e)

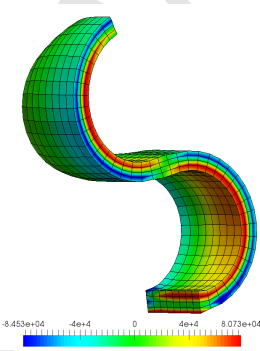

(h)

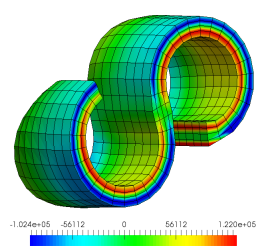

$(\mathrm{k})$

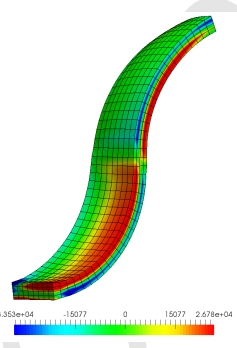

(c)

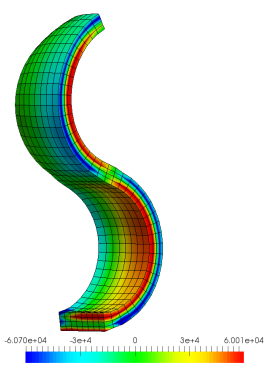

(f)

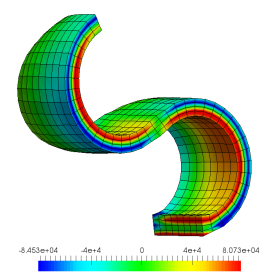

(i)

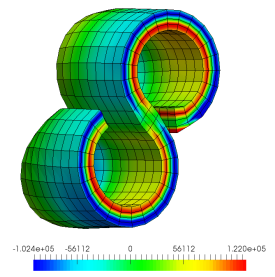

(l)

Figure 12: Bending actuator configuration 2. Contour plot of the pressure $p$ with the B-bar (with pressure smoothing) element for an applied surface electric charge $\omega_{0}=\lambda \times\left(3 \times 10^{-3}\right) Q / m^{2}$, for a value of the accumulated factor $\lambda$ of: (a) $\lambda=0.236$, (b) $\lambda=0.418$, (c) $\lambda=0.556$, (d) $\lambda=0.674$, (e) $\lambda=0.729$, (f) $\lambda=0.785$, (g) $\lambda=0.840$, (h) $\lambda=0.897$, (i) $\lambda=0.956$, (j) $\lambda=1.086$, (k) $\lambda=1.158$ and $\left(\mathrm{l}^{43} \lambda=1.239\right.$. Incompressible model in equation (86) using a discretisation of $40 \times 8 \times 4$ elements. 

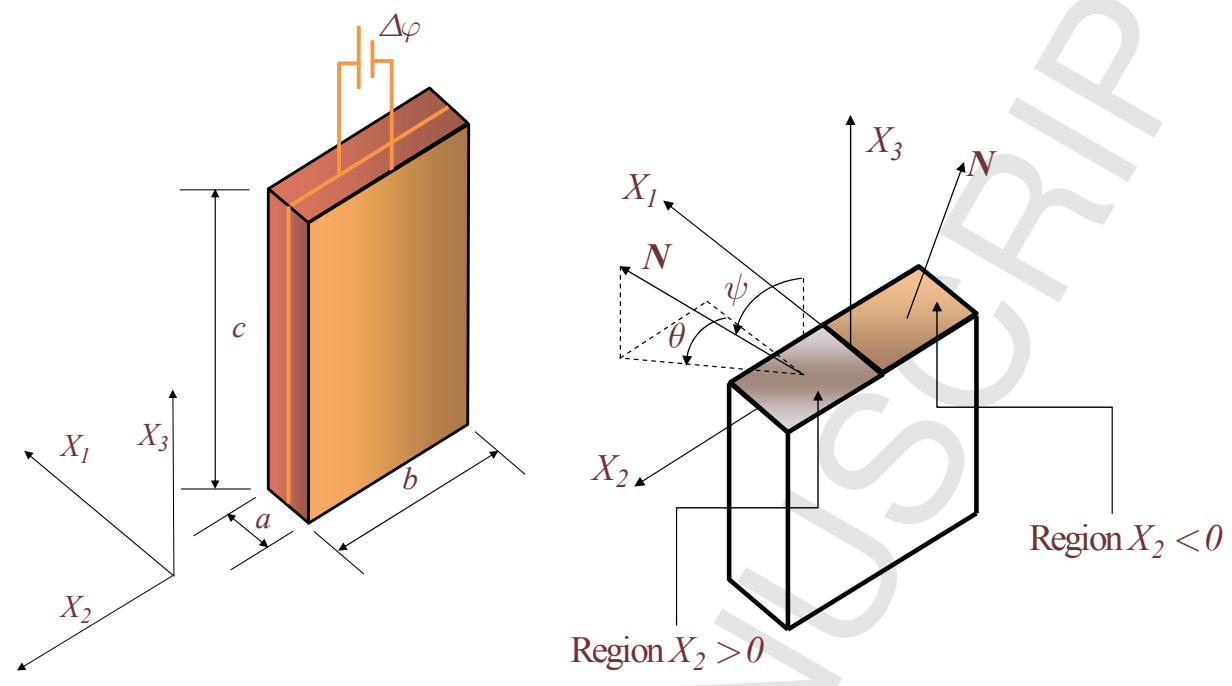

Figure 13: Torsional actuator. (a) Geometry and boundary conditions. (b) Arrangement of the fibres within the isotropic matrix. Illustration of spherical parametrisation of the vector $\boldsymbol{N}$ in the region $X_{2}>0$.

with $W_{\text {matrix }}$ defined as in (15) and with $W_{\text {fibre }}(\boldsymbol{F}, \boldsymbol{H}, J, \boldsymbol{N})$ defined as

$$
W_{\text {fibre }}(\boldsymbol{F}, \boldsymbol{H}, J, \boldsymbol{N})=\mu_{3} I I_{\boldsymbol{F} \boldsymbol{N}}+\mu_{3} I I_{\boldsymbol{H N}}-2 \mu_{3} \ln J .
$$

Notice that for the above definition of the internal energy (89) and (90), the Kirchhoff stress tensor associated fibre contribution will not be traceless. Hence, this fibre contribution of the energy functional will contribute to the pressure of the constitutive model. As a result, the variable $p$ in the various mixed variational principles described in Section 3 does not correspond to the hydrostatic pressure for this case. Making use of the relationship between both the Cauchy stress tensor $\boldsymbol{\sigma}$ and the Kirchhoff stress tensor $\boldsymbol{\tau}$, namely $\boldsymbol{\sigma}=J^{-1} \boldsymbol{\tau}$ and considering the definition of $\boldsymbol{\tau}$ in equation (14), the hydrostatic pressure for this particular example has to be computed as

$$
p_{\text {total }}=p+J^{-1}\left(\frac{\partial W_{\text {fibre }}}{\partial \boldsymbol{F}} \boldsymbol{F}^{T}+\left(\frac{\partial W_{\text {fibre }}}{\partial \boldsymbol{H}} \boldsymbol{H}^{T}\right) \times \boldsymbol{I}+\frac{\partial W_{\text {fibre }}}{\partial J} \boldsymbol{I}\right) .
$$

Figure 14 presents a comparison for the $\mathbf{M W F}, \mathbf{P} 2 \mathbf{P} 1, \mathbf{B}-$ bar and P1P1 elements for the Jacobian variable $J$, the hydrostatic pressure $p$ and the 


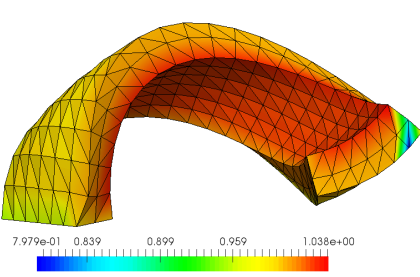

(a)

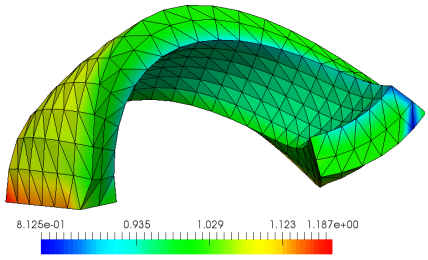

(d)

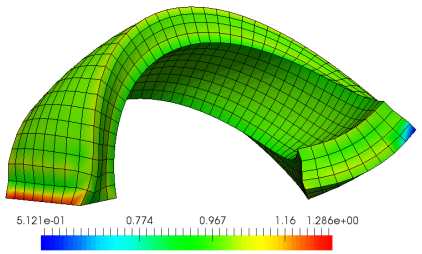

(g)

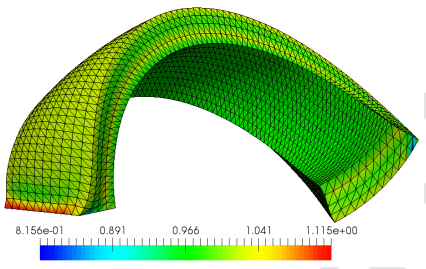

(j)

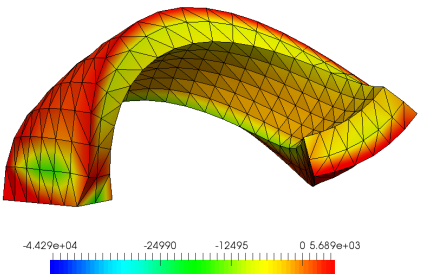

(b)

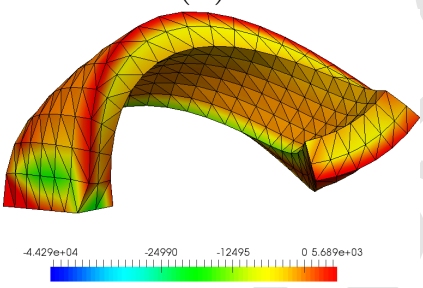

(e)

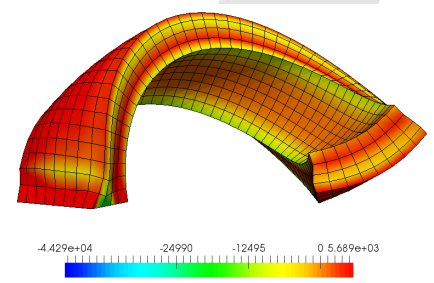

(h)

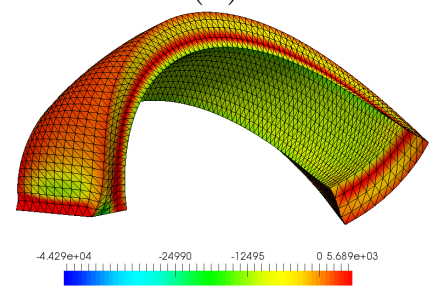

(k)

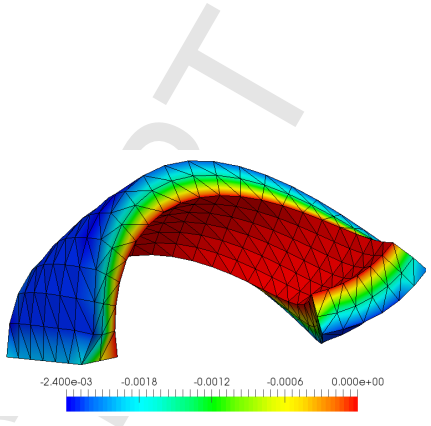

(c)

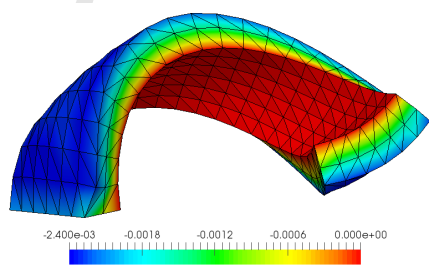

(f)

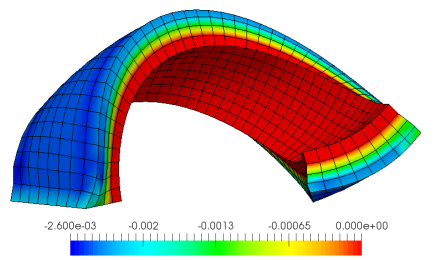

(i)

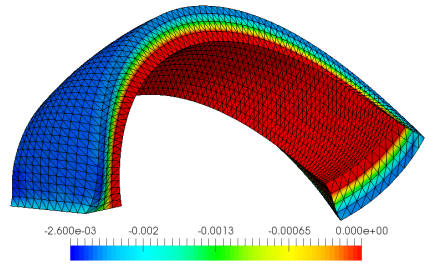

(l)

Figure 14: Torsional actuator. Contour plot of Jacobian variable $J$ (left column), hydrostatic pressure $p$ (center column) and the electric displacement field component $\boldsymbol{D}_{0_{1}}$ for (a), (b), (c) the $\mathbf{M} \hat{\mathbf{W}} \mathbf{F}$ element; (d), (e), (f) the P2P1 element; (g), (h), (i) the B-bar element and (j), (k), (l), the P1P1 element. Incompressible constitutive model in equation (89). Results obtained for a value of the applied surface electric charge of $\omega_{0}=0.0024 \mathrm{Q} / \mathrm{m}^{2}$. Results for a discretisation of $(2 \times 6 \times 20) \times 6$ elements for the $\mathbf{M} \hat{\mathbf{W}} \mathbf{F}$ and P2P1 elements; $4 \times 8 \times 40$ for the B-bar element and $(4 \times 10 \times 80) \times 6$ for the $\mathbf{P} 1 \mathbf{P} 1$ element. 


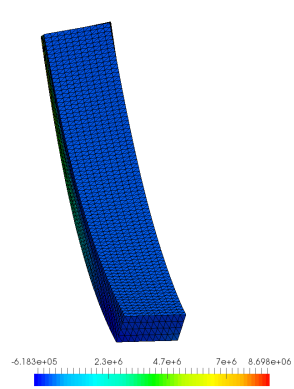

(a)

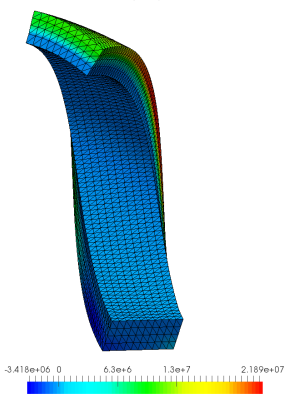

(d)

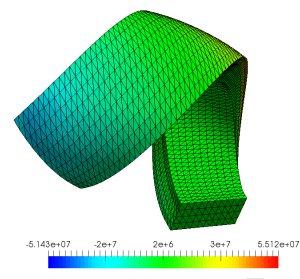

(g)

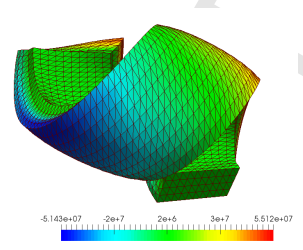

(j)

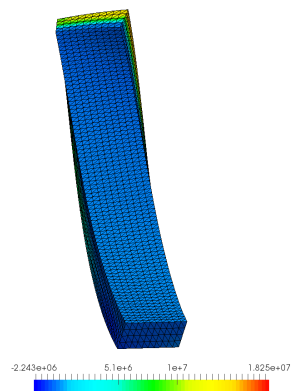

(b)

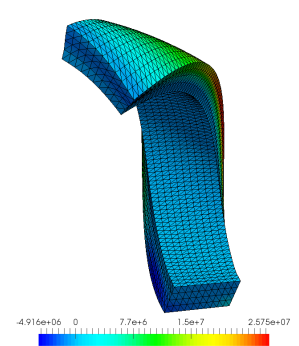

(e)

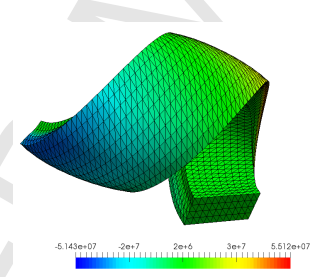

(h)

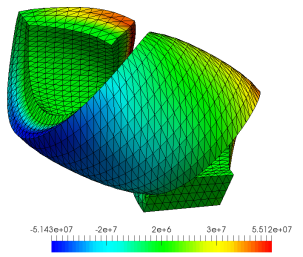

(k)

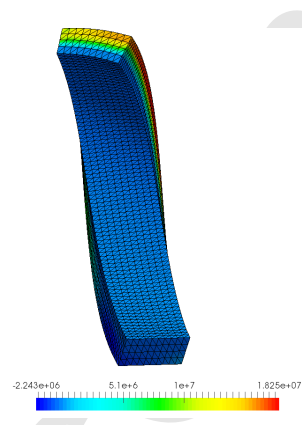

(c)

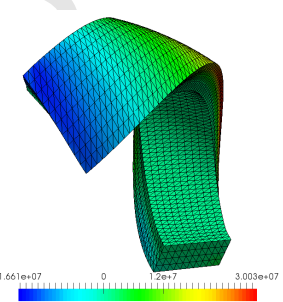

(f)

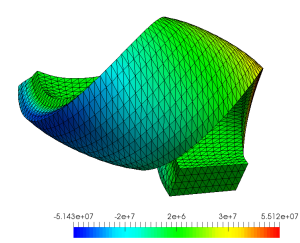

(i)

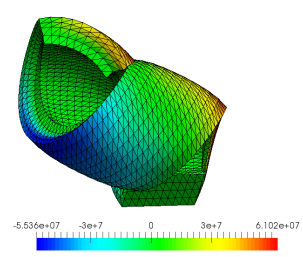

(1)

Figure 15: Torsional actuator. Contour plot of the Eulerian electric field $\boldsymbol{E}_{2}$ with the P1P1 (with $\alpha=0.1$ ) element for an applied surface electric charge $\omega_{0}=\lambda \times\left(3 \times 10^{-3}\right) Q / m^{2}$, for a value of the accumulated factor $\lambda$ of: (a) $\lambda=0.295$, (b) $\lambda=0.393$, (c) $\lambda=0.485$, (d) $\lambda=0.576$, (e) $\lambda=0.673$, (f) $\lambda=0.779$, (g) $\lambda=0.903$, (h) $\lambda=1.055$, (i) $\lambda=1.197$, (j) $\lambda=1.318$, (k) $\lambda=1.430$ and (l) $\lambda=1.562$. Incomp 4 sssible model in equation (89) using a discretisation of $4 \times 10 \times 80$ elements. 
electric displacement field $\boldsymbol{D}_{0_{1}}$. All the results in this Figure have been obtained for the same value of the applied surface electric charge $\omega_{0}$. A value of $\alpha=0.1$ has been chosen for the stabilisation parameter (refer to equation (49)) for the P1P1 element. A post-processing nodal average of the pressure (pressure smoothing) has been applied for the B-bar element, yielding a qualitatively good agreement between all the formulations for the three variables considered.

Finally, Figure 15 displays the contour plot of the Eulerian electric field variable $\boldsymbol{E}_{2}$ and the evolution of the deformation of the dielectric elastomer as the value of the applied surface electric charge $\omega_{0}$ increases, leading to the final extremely large deformation shown in Figure 15(1). These results have been obtained with the stabilised P1P1 element.

\section{Concluding remarks}

This paper has presented an extension of the variational and computational convex multi-variable framework for large strain/large electric field electro-elasticity developed by Gil and Ortigosa in References $[1,2,2]$ to nearly and truly incompressible scenarios. Following the ideas presented in Reference [1], the authors have shown some convexification or stabilisation techniques which enable to create convex multi-variable isochoric electromechanical invariants by modifying a priori non-convex multi-variable isochoric invariants.

Furthermore, an extended $\mathrm{Hu}$-Washizu mixed variational principle and its Finite Element computational implementation for nearly and truly incompressible scenarios has been presented in this work extending the ideas of the authors in References $[1,2]$. Additionally, a static condensation procedure has been applied in order to condense out the element-wise extra fields, leading to a computational cost comparable to that of the more standard three-field displacement-potential-pressure mixed formulation.

The computational framework for the standard three-field mixed variational principle in nearly and truly incompressible scenarios has also been presented within the context of multi-variable convexity. In addition, a Petrov-Galerkin stabilisation technique has been applied on the three-field formulation for the circumvention of the LBB condition, enabling the use of linear tetrahedral finite elements for the interpolation of the unknowns of the problem. Finally, a series of very challenging numerical examples have 
been presented in order to provide an exhaustive comparison of the different variational formulations and their different associated discretisations.

\section{Acknowledgements}

Both authors acknowledge the financial support provided by the Sêr Cymru National Research Network for Advanced Engineering and Materials. 


\section{Appendix A. Relationship between the Hessian operators of the extended internal energy $W(\mathcal{V})$ and that for the in- ternal energy $e\left(\nabla_{0} x, D_{0}\right)$}

The objective of this Section is to obtain a relationship between the deviatoric and volumetric components of the fourth order elasticity tensors $\hat{\mathcal{C}}$ and $\mathcal{C}_{p}$ (19), respectively, the third order piezoelectric tensor $\mathcal{Q}$ (19) and the second order dielectric tensor $\boldsymbol{\theta}$ (19) in terms of the components of the Hessian operator $\mathbb{H}_{\hat{W}}(22)$ and the first and second derivatives of the purely volumetric component of the internal energy $U(J)$.

\section{Appendix A.1. Elasticity tensor}

From equation (18), the second directional derivative of the internal energy $e$ with respect to changes of the geometry can be obtained as

$$
D^{2} e[\delta \boldsymbol{u} ; \boldsymbol{u}]=\nabla_{0} \delta \boldsymbol{u}: \hat{\mathcal{C}}: \nabla_{0} \boldsymbol{u}+\nabla_{0} \delta \boldsymbol{u}: \mathcal{C}_{p}: \nabla_{0} \boldsymbol{u}
$$

Comparison of equations (A.1) and (20) $)_{a}$ enables the deviatoric component of the elasticity tensor $\hat{\mathcal{C}}$ to be alternatively re-written in terms of the derivatives of the electro-kinematic variable set $\mathcal{V}$ as

$$
\begin{aligned}
\hat{\mathcal{C}} & =\hat{W}_{\boldsymbol{F} \boldsymbol{F}}+\boldsymbol{F} \times\left(\hat{W}_{\boldsymbol{H} \boldsymbol{H}} \times \boldsymbol{F}\right)+\hat{W}_{J J} \boldsymbol{H} \otimes \boldsymbol{H}+\mathcal{C}_{1} \\
& +2\left(\hat{W}_{\boldsymbol{F} \boldsymbol{H}} \times \boldsymbol{F}\right)^{\mathrm{sym}}+2\left(\hat{W}_{\boldsymbol{F} J} \otimes \boldsymbol{H}\right)^{\mathrm{sym}}+2\left(\hat{W}_{\boldsymbol{F} \boldsymbol{d}} \otimes \boldsymbol{D}_{0}\right)^{\mathrm{sym}} \\
& +2\left(\left(\boldsymbol{F} \times \hat{W}_{\boldsymbol{H} J}\right) \otimes \boldsymbol{H}\right)^{\mathrm{sym}}+2\left(\left(\boldsymbol{F} \times \hat{W}_{\boldsymbol{H} \boldsymbol{d}}\right) \otimes \boldsymbol{D}_{0}\right)^{\mathrm{sym}} \\
& +2\left(\boldsymbol{H} \otimes\left(\hat{W}_{J \boldsymbol{d}} \otimes \boldsymbol{D}_{0}\right)\right)^{\mathrm{sym}}+\mathcal{A},
\end{aligned}
$$

where

$$
\mathcal{A}_{i I j J}=\mathcal{E}_{i j p} \mathcal{E}_{I J P}\left(\boldsymbol{\Sigma}_{\boldsymbol{H}}+\hat{\Sigma}_{J} \boldsymbol{F}\right)_{p P} ; \quad \hat{\Sigma}_{J}=\frac{\partial \hat{W}}{\partial J} ; \quad \mathcal{C}_{1, i I j J}=\left(\hat{W}_{\boldsymbol{d d}}\right)_{i j} D_{0 I} D_{0 J} .
$$

Moreover, for any fourth order tensor $\mathcal{T}$ included in equation (A.2), the symmetrised tensor $\mathcal{T}^{\text {sym }}$ is defined as $\mathcal{T}_{i I j J}^{\text {sym }}=\frac{1}{2}\left(\mathcal{T}_{i I j J}+\mathcal{T}_{i J j I}\right)$.

For the volumetric component of the elasticity tensor $\mathcal{C}_{p}$, following a similar procedure, comparison of equations (A.1) and $(20)_{b}$ enables the following relationship for $\mathcal{C}_{p}$ in terms of the first and second derivatives of the volumetric energy $U(J)$ to be obtained as

$$
\mathcal{C}_{p}=U^{\prime \prime} \boldsymbol{H} \otimes \boldsymbol{H}+\mathcal{B} ; \quad \mathcal{B}_{i I j J}=U^{\prime}(J) \mathcal{E}_{i j p} \mathcal{E}_{I J P} F_{p P} .
$$




\section{Appendix A.2. Piezoelectric tensor}

From equation (18), the second directional derivative of the internal energy $e$ with respect to changes in geometry and electric displacement field leads to the following expression

$$
D^{2} e\left[\delta \boldsymbol{u} ; \Delta \boldsymbol{D}_{0}\right]=\left(\nabla_{0} \delta \boldsymbol{u}: \mathcal{Q}^{T}\right) \cdot \Delta \boldsymbol{D}_{0} .
$$

Comparison of equations (A.5) and $(20)_{a}$ allows to re-express the piezoelectric tensor $\mathcal{Q}^{T}$ in terms of the elements of the set $\mathcal{V}$ as

$$
\begin{aligned}
\mathcal{Q}^{T} & =\hat{W}_{\boldsymbol{F} \boldsymbol{D}_{0}}+\boldsymbol{F} \times \hat{W}_{\boldsymbol{H} \boldsymbol{D}_{0}}+\boldsymbol{H} \otimes \hat{W}_{J \boldsymbol{D}_{0}}+\boldsymbol{\mathcal { Q }}_{1}^{T} \\
& +\mathcal{Q}_{2}^{T}+\mathcal{Q}_{3}^{T}+\mathcal{Q}_{4}^{T}+\mathcal{Q}_{5}^{T}+\boldsymbol{\Sigma}_{\boldsymbol{d}} \otimes \boldsymbol{I} .
\end{aligned}
$$

where the expressions for the tensors $\mathcal{Q}_{i}^{T}$ in above equation (A.6) are given as

$$
\begin{aligned}
& \left(\mathcal{Q}_{1}^{T}\right)_{i I J}=\left(\hat{W}_{\boldsymbol{d} \boldsymbol{D}_{0}}\right)_{i J} D_{0 I} ; \\
& \left(\boldsymbol{\mathcal { Q }}_{2}^{T}\right)_{i I J}=\left(\hat{W}_{\boldsymbol{F d}}\right)_{i I j} F_{j J} ; \\
& \left(\mathcal{Q}_{3}^{T}\right)_{i I J}=\left(\boldsymbol{F} \times \hat{W}_{\boldsymbol{H} \boldsymbol{d}}\right)_{i I j} F_{j J} \\
& \left(\mathcal{Q}_{4}^{T}\right)_{i I J}=\left(\boldsymbol{H} \otimes \hat{W}_{J \boldsymbol{d} d}\right)_{i I j} F_{j J} ; \\
& \left(\mathcal{Q}_{5}^{T}\right)_{i I J}=\left(\hat{W}_{\boldsymbol{d} \boldsymbol{d}}\right)_{i j} F_{j J} D_{0 I}
\end{aligned}
$$

\section{Appendix A.3. Dielectric tensor}

The second directional derivative of the internal energy $e$ with respect to changes in the electric displacement field can be identified from equation (18) as

$$
D^{2} e\left[\delta \boldsymbol{D}_{0} ; \Delta \boldsymbol{D}_{0}\right]=\delta \boldsymbol{D}_{0} \cdot \boldsymbol{\theta} \Delta \boldsymbol{D}_{0} .
$$

Comparison of equations (A.8) and (20) $)_{a}$ enables the inverse of the dielectric tensor $\boldsymbol{\theta}$ to be re-expressed in terms of the elements of the set $\mathcal{V}$ as

$$
\boldsymbol{\theta}=\hat{W}_{\boldsymbol{D}_{0} \boldsymbol{D}_{0}}+\left(\hat{W}_{\boldsymbol{D}_{0} \boldsymbol{d}} \boldsymbol{F}+\boldsymbol{F}^{T} \hat{W}_{\boldsymbol{d} \boldsymbol{D}_{0}}\right)+\boldsymbol{F}^{T} \hat{W}_{\boldsymbol{d d}} \boldsymbol{F}
$$




\section{Appendix B. Relationships between the Hessian operator of the Helmholtz's energy functional and that for the in- ternal energy}

When an explicit representation of the Helmholtz's energy functional introduced in Section 2.6, namely $\Phi\left(\boldsymbol{\nabla}_{0} \boldsymbol{x},-\boldsymbol{\nabla}_{0} \varphi\right)(27)$ is not available, the associated Hessian operators need to be obtained in terms of the components of the Hessian operators associated to the internal energy function $e\left(\boldsymbol{\nabla}_{0} \boldsymbol{x}, \boldsymbol{D}_{0}\right)$, by exploiting the partial Legendre transforms presented in equation (27), respectively.

Following Gil and Ortigosa [2], the purely electric constitutive tensors $\varepsilon$ (32) and $\boldsymbol{\theta}$ (19) can be related as

$$
\varepsilon=\boldsymbol{\theta}^{-1}
$$

The expression relating the coupled contributions of both Hessian operators, namely $\mathcal{P}(32)$ and $\mathcal{Q}(19)$ yields

$$
-\mathcal{P}^{T}=\mathcal{Q}^{T} \bullet \boldsymbol{\varepsilon},
$$

where the operation $\bullet$ in above (B.2) indicates the contraction of the last and first components of the tensors on the left and right hand sides of the operation symbol $\bullet$, respectively. Finally, both deviatoric and volumetric contributions of the mechanical component of the Hessian operator of the Helmholtz energy, namely $\hat{\mathcal{C}}^{\star}$ (19) can be obtained as

$$
\hat{\mathcal{C}}^{\star}=\hat{\mathcal{C}}+\mathcal{Q} \bullet \mathcal{P} ; \quad \mathcal{C}_{p}^{\star}=\mathcal{C}_{p}
$$

with $\hat{\mathcal{C}}$ and $\mathcal{C}_{p}$ defined in (19). 


\section{Appendix C. Proof of convexity of stabilised invariants}

Appendix C.1. Stabilisation strategy 1 in Section 4.1

Convexity of invariant $W_{\hat{\boldsymbol{F}} \boldsymbol{D}_{0}}\left(\boldsymbol{F}, J, \boldsymbol{D}_{0}\right)$ in equation (45) is subject to positiveness of the variable $\mathcal{F}$ defined as

$$
\begin{aligned}
\mathcal{F}=D^{2} W_{\hat{\boldsymbol{F}} \boldsymbol{D}_{0}}\left[\delta \boldsymbol{F}, \delta J, \delta \boldsymbol{D}_{0} ; \delta \boldsymbol{F}, \delta J, \delta \boldsymbol{D}_{0}\right] & =D^{2} W_{\hat{\boldsymbol{F}} \boldsymbol{D}_{0}}[\delta \boldsymbol{F} ; \delta \boldsymbol{F}]+D^{2} W_{\hat{\boldsymbol{F}} \boldsymbol{D}_{0}}[\delta J ; \delta J] \\
& +D^{2} W_{\hat{\boldsymbol{F}} \boldsymbol{D}_{0}}\left[\delta \boldsymbol{D}_{0} ; \delta \boldsymbol{D}_{0}\right]+2 D^{2} W_{\hat{\boldsymbol{F}} \boldsymbol{D}_{0}}[\delta \boldsymbol{F} ; \delta J] \\
& +2 D^{2} W_{\hat{\boldsymbol{F}} \boldsymbol{D}_{0}}\left[\delta \boldsymbol{F} ; \delta \boldsymbol{D}_{0}\right]+2 D^{2} W_{\hat{\boldsymbol{F}} \boldsymbol{D}_{0}}\left[\delta J ; \delta \boldsymbol{D}_{0}\right],
\end{aligned}
$$

or equivalently,

$$
\mathcal{F}=\left[\begin{array}{lll}
\delta \boldsymbol{F}: & \delta J & \delta \boldsymbol{D}_{0} \cdot
\end{array}\right]\left[\begin{array}{ccc}
\frac{\partial^{2} W_{\hat{\boldsymbol{F}} \boldsymbol{D}_{0}}}{\partial \boldsymbol{F} \partial \boldsymbol{F}} & \frac{\partial^{2} W_{\hat{\boldsymbol{F}} \boldsymbol{D}_{0}}}{\partial \boldsymbol{F} \partial J} & \frac{\partial^{2} W_{\hat{\boldsymbol{F}} \boldsymbol{D}_{0}}}{\partial \boldsymbol{F} \partial \boldsymbol{D}_{0}} \\
\frac{\partial^{2} W_{\hat{\boldsymbol{F}} \boldsymbol{D}_{0}}}{\partial J \partial \boldsymbol{F}} & \frac{\partial^{2} W_{\hat{\boldsymbol{F}} \boldsymbol{D}_{0}}}{\partial J \partial J} & \frac{\partial^{2} W_{\hat{\boldsymbol{F}} \boldsymbol{D}_{0}}}{\partial J \partial \boldsymbol{D}_{0}} \\
\frac{\partial^{2} W_{\hat{\boldsymbol{F}} \boldsymbol{D}_{0}}}{\partial \boldsymbol{D}_{0} \partial \boldsymbol{F}} & \frac{\partial^{2} W_{\hat{\boldsymbol{F}} \boldsymbol{D}_{0}}}{\partial \boldsymbol{D}_{0} \partial J} & \frac{\partial^{2} W_{\hat{\boldsymbol{F}} \boldsymbol{D}_{0}}}{\partial \boldsymbol{D}_{0} \partial \boldsymbol{D}_{0}}
\end{array}\right]\left[\begin{array}{c}
: \delta \boldsymbol{F} \\
\delta J \\
\delta \boldsymbol{D}_{0}
\end{array}\right]
$$

Each of the terms featuring in (C.2) are obtained as

$$
\begin{aligned}
D^{2} W_{\hat{\boldsymbol{F}} \boldsymbol{D}_{0}}[\delta \boldsymbol{F} ; \delta \boldsymbol{F}] & =4\left(J^{-4 / 3} I I_{\boldsymbol{F}}+\gamma^{2} J^{-2 / 3} I I_{\boldsymbol{D}_{0}}\right) I I_{\delta \boldsymbol{F}}+8 J^{-4 / 3}(\delta \boldsymbol{F}: \boldsymbol{F})^{2} ; \\
D^{2} W_{\hat{\boldsymbol{F}} \boldsymbol{D}_{0}}[\delta J ; \delta J] & =\frac{28}{9} J^{-10 / 3} \delta J^{2} I I_{\boldsymbol{F}}^{2}+\frac{20}{9} \gamma^{2} J^{-8 / 3} \delta J^{2} I I_{\boldsymbol{F}} I I_{\boldsymbol{D}_{0}} ; \\
D^{2} W_{\hat{\boldsymbol{F}} \boldsymbol{D}_{0}}\left[\delta \boldsymbol{D}_{0} ; \delta \boldsymbol{D}_{0}\right] & =8 \gamma^{4}\left(\delta \boldsymbol{D}_{0} \cdot \boldsymbol{D}_{0}\right)^{2}+4 \gamma^{2}\left(J^{-2 / 3} I I_{\boldsymbol{F}}+\gamma^{2} I I_{\boldsymbol{D}_{0}}\right) I I_{\delta \boldsymbol{D}_{0}} \\
D^{2} W_{\hat{\boldsymbol{F}} \boldsymbol{D}_{0}}[\delta \boldsymbol{F} ; \delta J] & =-\frac{16}{3} J^{-7 / 3} \delta J I I_{\boldsymbol{F}}(\delta \boldsymbol{F}: \boldsymbol{F})-\frac{8}{3} \gamma^{2} J^{-5 / 3} \delta J I I_{\boldsymbol{D}_{0}}(\delta \boldsymbol{F}: \boldsymbol{F}) ; \\
D^{2} W_{\hat{\boldsymbol{F}} \boldsymbol{D}_{0}}\left[\delta \boldsymbol{F} ; \delta \boldsymbol{D}_{0}\right] & =8 \gamma^{2} J^{-2 / 3}(\delta \boldsymbol{F}: \boldsymbol{F})\left(\delta \boldsymbol{D}_{0} \cdot \boldsymbol{D}_{0}\right) ; \\
D^{2} W_{\hat{\boldsymbol{F}} \boldsymbol{D}_{0}}\left[\delta J ; \delta \boldsymbol{D}_{0}\right] & =-\frac{8}{3} \gamma^{2} J^{-5 / 3} \delta J I I_{\boldsymbol{F}}\left(\delta \boldsymbol{D}_{0} \cdot \boldsymbol{D}_{0}\right)
\end{aligned}
$$


Introduction of above equation (C.3) into (C.2) yields

$$
\begin{aligned}
& \mathcal{F}=\underbrace{8 J^{-4 / 3}(\delta \boldsymbol{F}: \boldsymbol{F})^{2}+4 J^{-4 / 3} I I_{\boldsymbol{F}} I I_{\delta \boldsymbol{F}}}_{\mathcal{F}_{1}}+\underbrace{4 \gamma^{2} J^{-2 / 3} I I_{\boldsymbol{D}_{0}} I I_{\delta \boldsymbol{F}}}_{\mathcal{F}_{2}} \\
& +\underbrace{8 \gamma^{4}\left(\delta \boldsymbol{D}_{0} \cdot \boldsymbol{D}_{0}\right)^{2}+4 \gamma^{4} I I_{\boldsymbol{D}_{0}} I I_{\delta \boldsymbol{D}_{0}}}_{\mathcal{F}_{3}}+\underbrace{\frac{28}{9} J^{-10 / 8} I I_{\boldsymbol{F}}^{2} \delta J^{2}}_{\mathcal{F}_{4}}+\underbrace{\frac{20}{9} \gamma^{2} J^{-8 / 3} I I_{\boldsymbol{F}} I I_{\boldsymbol{D}_{0}} \delta J^{2}}_{\mathcal{F}_{5}} \\
& -\underbrace{\frac{32}{3} J^{-7 / 3} \delta J I I_{\boldsymbol{F}}(\boldsymbol{F}: \delta \boldsymbol{F})}_{\mathcal{F}_{6}}-\underbrace{\frac{16}{3} \gamma^{2} J^{-5 / 3} \delta J I I_{\boldsymbol{D}_{0}}(\boldsymbol{F}: \delta \boldsymbol{F})}_{\mathcal{F}_{7}}+\underbrace{16 \gamma^{2} \delta J^{-2 / 3}(\boldsymbol{F}: \delta \boldsymbol{F})\left(\delta \boldsymbol{D}_{0} \cdot \boldsymbol{D}_{0}\right)}_{\mathcal{F}_{8}} \\
& -\underbrace{\frac{16}{3} \gamma^{2} J^{-5 / 3} \delta J I I_{\boldsymbol{F}}\left(\delta \boldsymbol{D}_{0} \cdot \boldsymbol{D}_{0}\right)}_{\mathcal{F}_{9}}+\underbrace{4 \gamma^{2} J^{-2 / 3} I I_{\boldsymbol{F}} I I_{\delta \boldsymbol{D}_{0}}}_{\mathcal{F}_{10}}
\end{aligned}
$$

Application of the Cauchy-Schwarz inequality to the tensors $\boldsymbol{F}$ and $\delta \boldsymbol{F}$ and to the vectors $\boldsymbol{D}_{0}$ and $\delta \boldsymbol{D}_{0}$ reads as,

$$
I I_{\boldsymbol{F}} I I_{\delta \boldsymbol{F}} \geq(\delta \boldsymbol{F}: \boldsymbol{F})^{2} ; \quad I I_{\boldsymbol{D}_{0}} I I_{\delta \boldsymbol{D}_{0}} \geq\left(\delta \boldsymbol{D}_{0} \cdot \boldsymbol{D}_{0}\right)^{2} .
$$

Equation (C.5) enables a set of inequalities for $\mathcal{F}_{1}$ and $\mathcal{F}_{3}$ in (C.4) to be written as,

$$
\begin{aligned}
& \mathcal{F}_{1}=8 J^{-4 / 3}(\delta \boldsymbol{F}: \boldsymbol{F})^{2}+4 J^{-4 / 3} I I_{\boldsymbol{F}} I I_{\delta \boldsymbol{F}} \geq 12 J^{-4 / 3}(\delta \boldsymbol{F}: \boldsymbol{F})^{2} ; \\
& \mathcal{F}_{3}=8 \gamma^{4}\left(\delta \boldsymbol{D}_{0} \cdot \boldsymbol{D}_{0}\right)^{2}+4 \gamma^{4} I I_{\boldsymbol{D}_{0}} I I_{\delta \boldsymbol{D}_{0}} \geq 12 \gamma^{4}\left(\delta \boldsymbol{D}_{0} \cdot \boldsymbol{D}_{0}\right)^{2} .
\end{aligned}
$$

Introduction of the inequalities for $\mathcal{F}_{1}$ and $\mathcal{F}_{3}$ in (C.6) into equation (C.4) and consideration of $\mathcal{F}_{10} \geq 0$ yields the following inequality

$$
\begin{aligned}
\mathcal{F} \geq & =\underbrace{12 J^{-4 / 3}(\delta \boldsymbol{F}: \boldsymbol{F})^{2}}_{\mathcal{F}_{1}^{\star}}+\underbrace{4 \gamma^{2} J^{-2 / 3} I I_{\boldsymbol{D}_{0}} I I_{\delta \boldsymbol{F}}}_{\mathcal{F}_{2}}+\underbrace{12 \gamma^{4}\left(\delta \boldsymbol{D}_{0} \cdot \boldsymbol{D}_{0}\right)^{2}}_{\mathcal{F}_{3}^{\star}} \\
& +\underbrace{\frac{28}{9} J^{-10 / 8} I I_{\boldsymbol{F}}^{2} \delta J^{2}}_{\mathcal{F}_{5}}+\underbrace{\frac{20}{9} \gamma^{2} J^{-8 / 3} I I_{\boldsymbol{F}} I I_{\boldsymbol{D}_{0}} \delta J^{2}}_{\mathcal{F}_{7}}-\underbrace{\frac{32}{3} J^{-7 / 3} \delta J I I_{\boldsymbol{F}}(\boldsymbol{F}: \delta \boldsymbol{F})}_{\mathcal{F}_{6}} \\
& -\underbrace{\frac{16}{3} \gamma^{2} J^{-5 / 3} \delta J I I_{\boldsymbol{D}_{0}}(\boldsymbol{F}: \delta \boldsymbol{F})}_{\mathcal{F}_{8}}+\underbrace{16 \gamma^{2} \delta J^{-2 / 3}(\boldsymbol{F}: \delta \boldsymbol{F})\left(\delta \boldsymbol{D}_{0} \cdot \boldsymbol{D}_{0}\right)}_{\mathcal{F}_{9}} \\
& -\underbrace{\frac{16}{3} \gamma^{2} J^{-5 / 3} \delta J I I_{\boldsymbol{F}}\left(\delta \boldsymbol{D}_{0} \cdot \boldsymbol{D}_{0}\right)} .
\end{aligned}
$$


Careful analysis of above terms $\mathcal{F}_{2}, \mathcal{F}_{5}$ and $\mathcal{F}_{7}$ yields the following inequality

$$
\mathcal{F}_{2}+\mathcal{F}_{5}+\mathcal{F}_{7}=J^{-8 / 3} \gamma^{2} I I_{\boldsymbol{D}_{0}} I I_{\boldsymbol{A}}+\frac{4}{9} \gamma^{2} J^{-8 / 3} I I_{\boldsymbol{F}} I I_{\boldsymbol{D}_{0}} \delta J^{2} \geq 0
$$

with $\boldsymbol{A}$ defined as $\boldsymbol{A}=\left(2 J \delta \boldsymbol{F}-\frac{4}{3} \delta J \boldsymbol{F}\right)$. Above inequality (C.8) enables to modify the inequality in equation (C.7) to give

$$
\begin{aligned}
\mathcal{F} \geq & =\underbrace{12 J^{-4 / 3}(\delta \boldsymbol{F}: \boldsymbol{F})^{2}}_{\mathcal{F}_{1}^{\star}}+\underbrace{12 \gamma^{4}\left(\delta \boldsymbol{D}_{0} \cdot \boldsymbol{D}_{0}\right)^{2}}_{\mathcal{F}_{3}^{\star}}+\underbrace{\frac{28}{9} J^{-10 / 8} I I_{\boldsymbol{F}}^{2} \delta J^{2}}_{\mathcal{F}_{4}}-\underbrace{\frac{32}{3} J^{-7 / 3} \delta J I I_{\boldsymbol{F}}(\boldsymbol{F}: \delta \boldsymbol{F})}_{\mathcal{F}_{6}} \\
& +\underbrace{16 \gamma^{2} \delta J^{-2 / 3}(\boldsymbol{F}: \delta \boldsymbol{F})\left(\delta \boldsymbol{D}_{0} \cdot \boldsymbol{D}_{0}\right)}_{\mathcal{F}_{8}}-\underbrace{\frac{16}{3} \gamma^{2} J^{-5 / 3} \delta J I I_{\boldsymbol{F}}\left(\delta \boldsymbol{D}_{0} \cdot \boldsymbol{D}_{0}\right)}_{\mathcal{F}_{9}} .
\end{aligned}
$$

Let us now consider the following auxiliary notation, i.e,

$$
a=J^{-2 / 3}(\delta \boldsymbol{F}: \boldsymbol{F}) ; \quad b=\gamma^{2}\left(\delta \boldsymbol{D}_{0} \cdot \boldsymbol{D}_{0}\right) ; \quad c=J^{-5 / 8} I I_{\boldsymbol{F}}
$$

With the help of the auxiliary notation in equation (C.10), above inequality (C.9) can be conveniently re-written as

$$
\mathcal{F} \geq=\underbrace{12 a^{2}}_{\mathcal{F}_{1}^{\star}}+\underbrace{12 b^{2}}_{\mathcal{F}_{3}^{\star}}+\underbrace{\frac{28}{9} c^{2}}_{\mathcal{F}_{4}}-\underbrace{\frac{32}{3} a c}_{\mathcal{F}_{6}}+\underbrace{16 a b}_{\mathcal{F}_{8}}-\underbrace{\frac{16}{3} b c}_{\mathcal{F}_{9}} \geq\left(4 a+2 b-\frac{4}{3} c\right)^{2} \geq 0 .
$$

Positiveness of $\mathcal{F}^{*}$ in above (C.11) yields positiveness of $\mathcal{F}$ in (C.7). Hence, the invariant $W_{\hat{\boldsymbol{F}} \boldsymbol{D}_{0}}\left(\boldsymbol{F}, J, \boldsymbol{D}_{0}\right)$ in equation (45) is convex with respect to its arguments, namely $\left\{\boldsymbol{F}, J, \boldsymbol{D}_{0}\right\}$.

Appendix C.2. Stabilisation strategy 2 in Section 4.2

Convexity of invariant $W_{\hat{\boldsymbol{H}} \boldsymbol{D}_{0}}$ in equation (47) is subject to positiveness of the variable $\mathcal{G}$ defined as

$$
\begin{aligned}
\mathcal{G} & =D^{2} W_{\hat{\boldsymbol{H}} \boldsymbol{D}_{0}}[\delta \boldsymbol{H} ; \delta \boldsymbol{H}]+D^{2} W_{\hat{\boldsymbol{H}} \boldsymbol{D}_{0}}[\delta J ; \delta J]+D^{2} W_{\hat{\boldsymbol{H}} \boldsymbol{D}_{0}}\left[\delta \boldsymbol{D}_{0} ; \delta \boldsymbol{D}_{0}\right] \\
& +2 D^{2} W_{\hat{\boldsymbol{H}} \boldsymbol{D}_{0}}[\delta \boldsymbol{H} ; \delta J]+2 D^{2} W_{\hat{\boldsymbol{H}} \boldsymbol{D}_{0}}\left[\delta \boldsymbol{H} ; \delta \boldsymbol{D}_{0}\right]+2 D^{2} W_{\hat{\boldsymbol{H}} \boldsymbol{D}_{0}}\left[\delta J ; \delta \boldsymbol{D}_{0}\right]
\end{aligned}
$$


where each of the terms on the right hand side of above equation (C.12) are defined as

$$
\begin{aligned}
D^{2} W_{\hat{\boldsymbol{H}} \boldsymbol{D}_{0}}[\delta \boldsymbol{H} ; \delta \boldsymbol{H}] & =24 J^{-4}(\delta \boldsymbol{H}: \boldsymbol{H})^{2} I I_{\boldsymbol{H}}+6 J^{-4} I I_{\boldsymbol{H}}^{2} I I_{\delta \boldsymbol{H}} \\
& +3 m \gamma^{2} J^{-2} I I_{\boldsymbol{H} \boldsymbol{D}_{0}}^{1 / 2} I I_{\delta \boldsymbol{H} \boldsymbol{D}_{0}}+3 m \gamma^{2} J^{-2} I I_{\boldsymbol{H} \boldsymbol{D}_{0}}^{1 / 2}\left(\boldsymbol{H} \boldsymbol{D}_{0} \cdot \delta \boldsymbol{H} \boldsymbol{D}_{0}\right)^{2} \\
D^{2} W_{\hat{\boldsymbol{H}} \boldsymbol{D}_{0}}[\delta J ; \delta J] & =20 J^{-6} \delta J^{2} I I_{\boldsymbol{H}}^{3}+6 m \gamma^{2} J^{-4} \delta J^{2} I I_{\boldsymbol{H} \boldsymbol{D}_{0}}^{3 / 2} ; \\
D^{2} W_{\hat{\boldsymbol{H}} \boldsymbol{D}_{0}}\left[\delta \boldsymbol{D}_{0} ; \delta \boldsymbol{D}_{0}\right] & =24 \gamma^{4}\left(\delta \boldsymbol{D}_{0} \cdot \boldsymbol{D}_{0}\right)^{2} I I_{\boldsymbol{D}_{0}}+6 \gamma^{4} I I_{\boldsymbol{D}_{0}}^{2} I I_{\delta \boldsymbol{D}_{0}} \\
& +3 m \gamma^{2} J^{-2} I I_{\boldsymbol{H} \boldsymbol{D}_{0}}^{1 / 2} I I_{\boldsymbol{H} \delta \boldsymbol{D}_{0}}+3 m \gamma^{2} J^{-2} I I_{\boldsymbol{H} \boldsymbol{D}_{0}}^{-1 / 2}\left(\boldsymbol{H} \boldsymbol{D}_{0} \cdot \boldsymbol{H} \delta \boldsymbol{D}_{0}\right)^{2} \\
D^{2} W_{\hat{\boldsymbol{H}} \boldsymbol{D}_{0}}[\delta \boldsymbol{H} ; \delta J] & =-24 J^{-5} \delta J(\delta \boldsymbol{H}: \boldsymbol{H}) I I_{\boldsymbol{H}}^{2}-6 m \gamma^{2} J^{-3} \delta J I I_{\boldsymbol{H} \boldsymbol{D}_{0}}^{1 / 2}\left(\boldsymbol{H} \boldsymbol{D}_{0} \cdot \delta \boldsymbol{H} \boldsymbol{D}_{0}\right) ; \\
D^{2} W_{\hat{\boldsymbol{H} \boldsymbol{D}_{0}}}\left[\delta \boldsymbol{H} ; \delta \boldsymbol{D}_{0}\right] & =3 m \gamma^{2} J^{-2} I I_{\boldsymbol{H} \boldsymbol{D}_{0}}^{-1 / 2}\left(\boldsymbol{H} \boldsymbol{D}_{0} \cdot \delta \boldsymbol{H} \boldsymbol{D}_{0}\right)\left(\boldsymbol{H} \boldsymbol{D}_{0} \cdot \boldsymbol{H} \delta \boldsymbol{D}_{0}\right) \\
& +3 m \gamma^{2} J^{-2} I I_{\boldsymbol{H} \boldsymbol{D}_{0}}\left(\left(\boldsymbol{H} \boldsymbol{D}_{0} \cdot \delta \boldsymbol{H} \delta \boldsymbol{D}_{0}\right)+\left(\boldsymbol{H} \delta \boldsymbol{D}_{0} \cdot \delta \boldsymbol{H} \boldsymbol{D}_{0}\right)\right) ; \\
D^{2} W_{\hat{\boldsymbol{H}} \boldsymbol{D}_{0}}\left[\delta J ; \delta \boldsymbol{D}_{0}\right] & =-6 m \gamma^{2} J^{-3} \delta J I I_{\boldsymbol{H} \boldsymbol{D}_{0}}^{1 / 2}\left(\boldsymbol{H} \boldsymbol{D}_{0} \cdot \boldsymbol{H} \delta \boldsymbol{D}_{0}\right),
\end{aligned}
$$

with $m=0.0513$. Introduction of all the terms in above equation (C.13) (and consideration of strictly the necessary unconditionally positive terms in above equation (C.13)) into the definition of $\mathcal{G}$ in (C.12) enables, after a careful inspection, to obtain the following properly arranged inequality for $\mathcal{G}$ as

$$
\begin{aligned}
& \mathcal{G} \geq \underbrace{24 J^{-4}(\delta \boldsymbol{H}: \boldsymbol{H})^{2} I I_{\boldsymbol{H}}+5.5385 J^{-4} I I_{\boldsymbol{H}}^{2} I I_{\delta \boldsymbol{H}}-48 J^{-5} \delta J(\delta \boldsymbol{H}: \boldsymbol{H}) I I_{\boldsymbol{H}}^{2}+19.5 J^{-6} \delta J^{2} I I_{\boldsymbol{H}}^{3}}_{\mathcal{G}_{1}} \\
& +\underbrace{6 m J^{-4} I I_{\boldsymbol{H}}^{2} I I_{\delta \boldsymbol{H}}+6 m \gamma^{2} J^{-2} I I_{\boldsymbol{H} \boldsymbol{D}_{0}}\left(\left(\boldsymbol{H} \boldsymbol{D}_{0} \cdot \delta \boldsymbol{H} \delta \boldsymbol{D}_{0}\right)+\left(\boldsymbol{H} \delta \boldsymbol{D}_{0} \cdot \delta \boldsymbol{H} \boldsymbol{D}_{0}\right)\right)+6 m \gamma^{4} I I_{\boldsymbol{D}_{0}}^{2} I I_{\delta \boldsymbol{D}_{0}}}_{\mathcal{G}_{2}} \\
& +\underbrace{3 m J^{-4} I I_{\boldsymbol{H}}^{2} I I_{\delta \boldsymbol{H}}+6 m \gamma^{2} J^{-2} I I_{\boldsymbol{H} \boldsymbol{D}_{0}}^{1 / 2}\left(\boldsymbol{H} \boldsymbol{D}_{0} \cdot \delta \boldsymbol{H} \boldsymbol{D}_{0}\right)\left(\boldsymbol{H} \boldsymbol{D}_{0} \cdot \boldsymbol{H} \delta \boldsymbol{D}_{0}\right)+3 m \gamma^{4} I I_{\boldsymbol{D}_{0}}^{2} I I_{\delta \boldsymbol{D}_{0}}}_{\mathcal{G}_{3}} \\
& +\underbrace{6 m J^{-6} \delta J^{2} I I_{\boldsymbol{H}}^{3}-12 m \gamma^{2} J^{-3} \delta J I I_{\boldsymbol{H} \boldsymbol{D}_{0}}^{1 / 2}\left(\boldsymbol{H} \boldsymbol{D}_{0} \cdot \boldsymbol{H} \delta \boldsymbol{D}_{0}\right)+6 m \gamma^{4} I I_{\boldsymbol{D}_{0}}^{2} I I_{\delta \boldsymbol{D}_{0}}}_{\mathcal{G}_{4}}] \\
& +\underbrace{3 m \gamma^{2} J^{-2} I I_{\boldsymbol{H} \boldsymbol{D}_{0}}^{1 / 2}\left(\boldsymbol{H} \boldsymbol{D}_{0} \cdot \delta \boldsymbol{H} \boldsymbol{D}_{0}\right)^{2}-6 m \gamma^{2} J^{-3} \delta J I I_{\boldsymbol{H} \boldsymbol{D}_{0}}^{1 / 2}\left(\boldsymbol{H} \boldsymbol{D}_{0} \cdot \delta \boldsymbol{H} \boldsymbol{D}_{0}\right)+3 m \gamma^{2} J^{-4} \delta J^{2} I I_{\boldsymbol{H} \boldsymbol{D}_{0}}^{3 / 2}}_{\mathcal{G}_{5}} \\
& +\underbrace{3 m \gamma^{2} J^{-2} I I_{\boldsymbol{H} \boldsymbol{D}_{0}}^{1 / 2} I I_{\delta \boldsymbol{H} \boldsymbol{D}_{0}}-6 m \gamma^{2} J^{-3} \delta J I I_{\boldsymbol{H} \boldsymbol{D}_{0}}^{1 / 2}\left(\boldsymbol{H} \boldsymbol{D}_{0} \cdot \delta \boldsymbol{H} \boldsymbol{D}_{0}\right)+3 m \gamma^{2} J^{-4} \delta J^{2} I I_{\boldsymbol{H} \boldsymbol{D}_{0}}^{3 / 2}}_{\mathcal{G}_{6}}
\end{aligned}
$$

The objective of the following derivations is to prove the positiveness of 
each of the terms $\mathcal{G}_{i}$, which would prove the convexity of the invariant $W_{\hat{\boldsymbol{H}} \boldsymbol{D}_{0}}$. Consideration of the Cauchy-Schwartz inequality enables to re-write the two first terms of $\mathcal{G}_{1}$ in (C.14) as

$$
24 J^{-4}(\delta \boldsymbol{H}: \boldsymbol{H})^{2} I I_{\boldsymbol{H}}+5.5385 J^{-4} I I_{\boldsymbol{H}}^{2} I I_{\delta \boldsymbol{H}} \geq 29.5385 J^{-4}(\delta \boldsymbol{H}: \boldsymbol{H})^{2} I I_{\boldsymbol{H}} .
$$

Introduction of the above inequality (C.15) in the definition of $\mathcal{G}_{1}$ enables to obtain the following inequality for $\mathcal{G}_{1}$ as

$$
\mathcal{G}_{1}=\frac{24 I I_{\boldsymbol{H}}}{J^{6}}\left(J(\delta \boldsymbol{H}: \boldsymbol{H})-I I_{\boldsymbol{H}} \delta J\right)^{2} \geq 0 .
$$

Regarding $\mathcal{G}_{2}$ (C.14), application of the Cauchy-Schwartz inequality on the first term of its definition in (C.14) enables to conveniently re-write this term as

$$
\begin{aligned}
6 m J^{-4} I I_{\boldsymbol{H}} I I_{\delta \boldsymbol{H}} & =6 m J^{-4} I I_{\boldsymbol{H}}^{2} I I_{\delta \boldsymbol{H}}\left(\frac{I I_{\boldsymbol{H} \boldsymbol{D}_{0}}}{I I_{\boldsymbol{H} \boldsymbol{D}_{0}}}\right)^{1 / 2} \geq 6 m J^{-4} \frac{I I_{\boldsymbol{H}}^{2} I I_{\delta \boldsymbol{H}} I I_{\boldsymbol{H} \boldsymbol{D}_{0}}^{1 / 2}}{I I_{\boldsymbol{H}}^{1 / 2} I I_{\boldsymbol{D}_{0}}^{1 / 2}} \\
& \geq 6 m J^{-4}\left(\frac{I I_{\boldsymbol{H}}}{I I_{\boldsymbol{D}_{0}}}\right)^{1 / 2} I I_{\boldsymbol{H} \boldsymbol{D}_{0}}^{1 / 2} \operatorname{tr}\left(\boldsymbol{H}^{T} \delta \boldsymbol{H} \delta \boldsymbol{H}^{T} \boldsymbol{H}\right)
\end{aligned}
$$

Similarly, the third term of $\mathcal{G}_{2}$ in (C.14) can be conveniently re-written as

$$
6 m \gamma^{4} I I_{\boldsymbol{D}_{0}}^{2} I I_{\delta \boldsymbol{D}_{0}} \geq 6 m \gamma^{4}\left(\frac{I I_{\boldsymbol{D}_{0}}}{I I_{\boldsymbol{H}}}\right)^{1 / 2} I I_{\boldsymbol{H} \boldsymbol{D}_{0}}^{1 / 2} I I_{\boldsymbol{D}_{0}} I I_{\delta \boldsymbol{D}_{0}}
$$

Consideration of the above inequalities in (C.17) and (C.18) enables to obtain the following inequality for $\mathcal{G}_{2}$,

$$
\mathcal{G}_{2} \geq 6 m I I_{\boldsymbol{H} \boldsymbol{D}_{0}}^{1 / 2} \operatorname{tr}\left(\boldsymbol{B}^{T} \boldsymbol{B}\right) \geq 0
$$

which is clearly greater than zero and with the second order tensor $\boldsymbol{B}$ defined as

$$
\boldsymbol{B}=\left(\frac{I I_{\boldsymbol{H}}}{I I_{\boldsymbol{D}_{0}}}\right)^{1 / 2} J^{-2} \delta \boldsymbol{H}^{T} \boldsymbol{H}+\gamma^{2}\left(\frac{I I_{\boldsymbol{D}_{0}}}{I I_{\boldsymbol{H}}}\right)^{1 / 2}\left(\delta \boldsymbol{D}_{0} \otimes \boldsymbol{D}_{0}\right)
$$

Let us consider now $\mathcal{G}_{3}$ in (C.14). The critical situation is obtained when the second term becomes negative (the first and third terms are always positive). In this critical situation, after recursive use of the Cauchy-Schwartz 
inequality it is possible to re-write this term as

$$
\begin{aligned}
-6 m \gamma^{2} J^{-2} \frac{\left|\left(\boldsymbol{H} \boldsymbol{D}_{0} \cdot \delta \boldsymbol{H} \boldsymbol{D}_{0}\right)\left(\boldsymbol{H} \boldsymbol{D}_{0} \cdot \boldsymbol{H} \delta \boldsymbol{D}_{0}\right)\right|}{I I_{\boldsymbol{H} \boldsymbol{D}_{0}}^{1 / 2}} \geq & -6 m \gamma^{2} J^{-2} \frac{I I_{\boldsymbol{H} \boldsymbol{D}_{0}} I I_{\delta \boldsymbol{H} \boldsymbol{D}_{0}}^{1 / 2} I I_{\boldsymbol{H} \delta \boldsymbol{D}_{0}}^{1 / 2}}{I I_{\boldsymbol{H}}^{1 / 2} I I_{\boldsymbol{D}_{0}}^{1 / 2}} \\
& -6 m \gamma^{2} J^{-2} \frac{I I_{\boldsymbol{H}}^{3 / 2} I I_{\boldsymbol{D}_{0}}^{3 / 2} I I_{\delta \boldsymbol{H}}^{1 / 2} I I_{\delta \boldsymbol{D}_{0}}^{1 / 2}}{I I_{\boldsymbol{H}}^{1 / 2} I I_{\boldsymbol{D}_{0}}^{1 / 2}} \\
& -6 m \gamma^{2} J^{-2} I I_{\boldsymbol{H}} I I_{\boldsymbol{D}_{0}} I I_{\delta \boldsymbol{H}}^{1 / 2} I I_{\delta \boldsymbol{D}_{0}}^{1 / 2}
\end{aligned}
$$

Consideration of the above inequality (C.21) enables to obtain the following inequality for $\mathcal{G}_{3}$ (C.14) as

$$
\mathcal{G}_{3} m \geq 3 J^{-4}\left(I I_{\boldsymbol{H}} I I_{\delta \boldsymbol{H}}^{1 / 2}-\gamma^{2} J^{-2} I I_{\boldsymbol{D}_{0}} I I_{\delta \boldsymbol{D}_{0}}^{1 / 2}\right)^{2} \geq 0
$$

which is clearly greater than zero. Let us now consider $\mathcal{G}_{4}$ in (C.14). Following a similar strategy to that in equation (C.21), it is possible to re-write the second term of $\mathcal{G}_{4}$ in the most critical case, namely, when it attains negative values, as

$$
-12 m \gamma^{2} J^{-3} \delta J I I_{\boldsymbol{H} \boldsymbol{D}_{0}}^{1 / 2}\left|\boldsymbol{H} \boldsymbol{D}_{0} \cdot \boldsymbol{H} \delta \boldsymbol{D}_{0}\right| \geq-12 m \gamma^{2} J^{-3} \delta J I I_{\boldsymbol{H}}^{3 / 2} I I_{\boldsymbol{D}_{0}} I I_{\delta \boldsymbol{D}_{0}}^{1 / 2},
$$

which enables to obtain the following inequality for $\mathcal{G}_{4}$ (C.14) as

$$
\mathcal{G}_{4} \geq 6 m J^{-6}\left(\delta J I I_{\boldsymbol{H}}^{3 / 2}-\gamma^{2} J^{3} I I_{\boldsymbol{D}_{0}} I I_{\delta \boldsymbol{D}_{0}}^{1 / 2}\right)^{2} \geq 0
$$

which is clearly greater than zero. Finally, it is possible to re-write both $\mathcal{G}_{5}$ and $\mathcal{G}_{6}$ as

$$
\begin{aligned}
& \mathcal{G}_{5}=3 m \gamma^{2} J^{-4} I I_{\boldsymbol{H} \boldsymbol{D}_{0}}^{1 / 2}\left(\delta J I I_{\boldsymbol{H} \boldsymbol{D}_{0}}+J\left(\boldsymbol{H} \boldsymbol{D}_{0} \cdot \delta \boldsymbol{H} \boldsymbol{D}_{0}\right)\right)^{2} \geq 0 \\
& \mathcal{G}_{6}=3 m \gamma^{2} J^{-4} I I_{\boldsymbol{H} \boldsymbol{D}_{0}}\left(J \delta \boldsymbol{H} \boldsymbol{D}_{0}+\delta J \boldsymbol{H} \boldsymbol{D}_{0}\right) \cdot\left(J \delta \boldsymbol{H} \boldsymbol{D}_{0}+\delta J \boldsymbol{H} \boldsymbol{D}_{0}\right) \geq 0
\end{aligned}
$$

with both inequalities being clearly greater than zero. Therefore, positiveness of all $\mathcal{G}_{i}$ (C.14) for $1 \leq i \leq 6$ proves the convexity of the invariant $W_{\hat{\boldsymbol{H}} \boldsymbol{D}_{0}}$ (47). 


\section{References}

[1] A. J. Gil, R. Ortigosa, A new framework for large strain electromechanics based on convex multi-variable strain energies: variational formulation and material characterisation, Computer Methods in Applied Mechanics and Engineering 302 (2016) 293-328.

[2] R. Ortigosa, A. J. Gil, A new framework for large strain electromechanics based on convex multi-variable strain energies: Finite Element discretisation and computational implementation, Computer Methods in Applied Mechanics and Engineering 302 (2016) 329-360.

[3] R. Ortigosa, A. J. Gil, A new framework for large strain electromechanics based on convex multi-variable strain energies: conservation laws and hyperbolicity, Computer Methods in Applied Mechanics and Engineering (Under review).

[4] R. E. Pelrine, R. D. Kornbluh, J. P. Joseph, Electrostriction of polymer dielectrics with compliant electrodes as a means of actuation, Sensors and Actuators A: Physical 64 (1998) 77-85.

[5] R. Pelrine, R. Kornbluh, Q. Pei, J. Joseph, High-speed electrically actuated elastomers with strain greater than $100 \%$, Science 287 (2000) 836-839.

[6] G. Kofod, P. Sommer-Larsen, R. Kornbluh, R. Pelrine, Actuation response of polyacrylate dielectric elastomers, Journal of Intelligent Material Systems and Structures 14 (2003) 787-793.

[7] R. Pelrine, R. D. Kornbluh, Q. Pei, S. Stanford, S. Oh, J. Eckerle, R. J. Full, M. A. Rosenthal, K. Meijer, Dielectric elastomer artificial muscle actuators: toward biomimetic motion, 2002.

[8] T. Li, C. Keplinger, R. Baumgartner, S. Bauer, W. Yang, Z. Suo, Giant voltage-induced deformation in dielectric elastomers near the verge of snap-through instability, Journal of the Mechanics and Physics of Solids 61 (2013) 611-628.

[9] S. Chiba, M. Waki, T. Wada, Y. Hirakawa, K. Masuda, T. Ikoma, Consistent ocean wave energy harvesting using electroactive polymer (dielectric elastomer) artificial muscle generators, Applied Energy 104 (2013) $497-502$. 
[10] S. Skatulla, C. Sansour, A. Arockiarajan, A multiplicative approach for nonlinear electro-elasticity, Computer Methods in Applied Mechanics and Engineering 245-246 (2012) 243-255.

[11] A. Dorfmann, R. W. Ogden, Nonlinear electroelasticity, Acta Mechanica 174 (2005) 167-183.

[12] A. Dorfmann, R. Ogden, Nonlinear electroelastic deformations, Journal of Elasticity 82 (2006) 99-127.

[13] R. Bustamante, A. Dorfmann, R. Ogden, Nonlinear electroelastostatics: a variational framework, Zeitschrift für angewandte Mathematik und Physik 60 (2009) 154-177.

[14] R. Bustamante, A. Dorfmann, R. Ogden, On electric body forces and Maxwell stresses in nonlinearly electroelastic solids, International Journal of Engineering Science 47 (2009) 1131-1141.

[15] R. Bustamante, Transversely isotropic non-linear electro-active elastomers, Acta Mechanica 206 (2009) 237-259.

[16] R. Bustamante, J. Merodio, Constitutive structure in coupled non-linear electro-elasticity: Invariant descriptions and constitutive restrictions, International Journal of Non-Linear Mechanics 46 (2011) 1315 - 1323.

[17] A. W. Richards, G. M. Odegard, Constitutive modeling of electrostrictive polymers using a hyperelasticity-based approach, Journal of Applied Mechanics 77 (2009).

[18] R. M. McMeeking, C. M. Landis, Electrostatic forces and stored energy for deformable dielectric materials, Journal of Applied Mechanics 72 (2008) 581-590.

[19] Z. Suo, X. Zhao, W. H. Greene, A nonlinear field theory of deformable dielectrics, Journal of the Mechanics and Physics of Solids 56 (2008) $467-486$.

[20] M. Itskov, V. N. Khiêm, A polyconvex anisotropic free energy function for electro- and magneto-rheological elastomers, Mathematics and Mechanics of Solids (2014) 1-12. 
[21] D. Vu, P. Steinmann, A 2-D coupled BEM-FEM simulation of electroelastostatics at large strain, Computer Methods in Applied Mechanics and Engineering 199 (2010) 1124-1133.

[22] D. Vu, P. Steinmann, On 3-D coupled BEM-FEM simulation of nonlinear electro-elastostatics, Computer Methods in Applied Mechanics and Engineering 201-204 (2012) 82-90.

[23] D. K. Vu, P. Steinmann, G. Possart, Numerical modelling of non-linear electroelasticity, International Journal for Numerical Methods in Engineering 70 (2007) 685-704.

[24] J. Zhou, W. Hong, X. Zhao, Z. Zhang, Z. Suo, Propagation of instability in dielectric elastomers, International Journal of Solids and Structures 45 (2008) 3739-3750.

[25] K. Bertoldi, M. Gei, Instabilities in multilayered soft dielectrics, Journal of the Mechanics and Physics of Solids 59 (2011) 18-42.

[26] M. H. Siboni, P. P. Castañeda, Fiber-constrained, dielectric-elastomer composites: Finite-strain response and stability analysis, Journal of the Mechanics and Physics of Solids 68 (2014) 211-238.

[27] M. H. Siboni, R. Avazmohammadi, P. P. Castañeda, Electromechanical instabilities in fiber-constrained, dielectric-elastomer composites subjected to all-around dead-loading, Mathematics and Mechanics of Solids (2014).

[28] C. Miehe, D. Vallicotti, D. Zäh, Computational structural and material stability analysis in finite electro-elasto-statics of electro-active materials, International Journal for Numerical Methods in Engineering 102 (2015) 1605-1637. Nme.4855.

[29] J. M. Ball, Convexity conditions and existence theorems in nonlinear elasticity, Archive for Rational Mechanics and Analysis 63 (1976) 337403.

[30] J. E. Marsden, T. J. R. Hughes, Mathematical foundations of elasticity, 1994. 
[31] A. Dorfmann, R. W. Ogden, Electroelastic waves in a finitely deformed electroactive material, IMA Journal of Applied Mathematics 75 (2010) 603-636.

[32] L. Sluys, Wave propagation, localisation and dispersion in softening solids, 1992.

[33] J. M. Ball, Energy-minimising configurations in nonlinear elasticity, Archive for Rational Mechanics and Analysis 63 (1976) 337-403.

[34] J. M. Ball, Geometry, Mechanics and Dynamics, Springer, pp. 3-59.

[35] J. M. Ball, F. Murat, $\mathrm{W}^{1, p}$-quasiconvexity and variational problems for multiple integrals, Journal of Functional Analysis 58 (1984) 225-253.

[36] B. Dacorogna, Direct Methods in the Calculus of Variations, Springer, 2008.

[37] P. Ciarlet, Existence theorems in intrinsic nonlinear elasticity, Journal des mathématiques pures et appliqués 94 (2010) 229-243.

[38] P. L. Tallec, Handbook of Numerical Analysis. Numerical Methods for Solids, North Holland.

[39] J. Schröder, P. Neff, D. Balzani, A variational approach for materially stable anisotropic hyperelasticity, International Journal of Solids and Structures 42 (2005) 4352-4371.

[40] J. Schröder, P. Neff, Invariant formulation of hyperelastic transverse isotropy based on polyconvex free energy functions, International Journal of Solids and Structures 40 (2003) 401-445.

[41] M. Aguirre, A. J. Gil, J. Bonet, A. A. Carreño, A vertex centred finite volume Jameson-Schmidt-Turkel (JST) algorithm for a mixed conservation formulation in solid dynamics, Journal of Computational Physics 259 (2014) 672-699.

[42] J. Schröder, P. Neff, V. Ebbing, Anisotropic polyconvex energies on the basis of crystallographic motived structural tensors, Journal of Mechanics and Physics of Solids 56 (2008) 3486-3506. 
[43] J. Schröder, P. Neff, V. Ebbing, Polyconvex energies for trigonal, tetragonal and cubic symmetry groups, in: IUTAM-Symposium on variational concepts with applications to the mechanics of materials: Proceedings of the IUTAM-Syposium.

[44] J. Schröder, P. Wriggers, D. Balzani, A new mixed finite element based on different approximations of the minors of deformation tensors, Computer Methods in Applied Mechanics and Engineering 200 (2011) 35833600 .

[45] J. Schröder, Anisotropic polyconvex energies, in: J. Schröder, P. Neff (Eds.), Poly-, quasi- and rank-one convexity in Applied Mechanics, volume 516 of CISM Courses and Lectures, volume 516 of CISM Courses and Lectures, Springer-Verlag, 2010, pp. 53-105.

[46] J. Bonet, A. J. Gil, R. Ortigosa, A computational framework for polyconvex large strain elasticity, Computer Methods in Applied Mechanics and Engineering 283 (2015) 1061-1094.

[47] A. J. Gil, C. H. Lee, J. Bonet, R. Ortigosa, A first order hyperbolic framework for large strain computational solid dynamics - Part II: Total Lagrangian compressible, nearly incompressible and truly incompressible elasticity, Computer Methods in Applied Mechanics and Engineering 300 (2016) 146-181.

[48] M. Itskov, N. Aksel, A class of orthotropic and transversely isotropic hyperelastic constitutive models based on a polyconvex strain energy function, International Journal of Solids and Structures 41 (2004) 38333848 .

[49] M. Itskov, A. Ehret, D. Mavrilas, A polyconvex anisotropic strainenergy function for soft collagenous tissues, Biomechanics and Modeling in Mechanobiology 5 (2006) 17-26.

[50] A. E. Ehret, M. Itskov, A polyconvex hyperelastic model for fiberreinforced materials in application to soft tissues, Journal of Materials Science 42 (2007) 8853-8863.

[51] R. Ortigosa, A. J. Gil, J. Bonet, C. Hesch, A computational framework for polyconvex large strain elasticity for geometrically exact beam theory, Computational Mechanics (2015). 
[52] J. Bonet, R. D. Wood, Nonlinear Continuum Mechanics for Finite Element Analysis, Cambridge University Press, second edition, 2008.

[53] A. J. Gil, C. H. Lee, J. Bonet, M. Aguirre, A stabilised Petrov-Galerkin formulation for linear tetrahedral elements in compressible, nearly incompressible and truly incompressible fast dynamics, Computer Methods in Applied Mechanics and Engineering 276 (2014) 659-690.

[54] F. Auricchio, L. B. de Veiga, C. Lovadina, A. Reali, A stability study of some mixed finite elements for large deformation elasticity problems, Computational Methods for Applied Mechanical Engineering 194 (2005) 1075-1092.

[55] F. Brezzi, M. Fortin, Mixed and Hybrid Finite Element Methods, Springer-Verlag New York, Inc., New York, NY, USA, 1991.

[56] I. Babuška, The finite element method with Lagrangian multipliers, Numerische Mathematik 20 (1973) 179-192.

[57] J. Bonet, A. J. Gil, C. H. Lee, M. Aguirre, R. Ortigosa, A first order hyperbolic framework for large strain computational solid dynamics Part I: Total Lagrangian isothermal elasticity, Computer Methods in Applied Mechanics and Engineering 283 (2015) 689-732.

[58] K. Izian, C. H. Lee, A. J. Gil, J. Bonet, A Two-Step Taylor-Galerkin method for explicit solid dynamics, Engineering Computations 31 (2013) 366-387.

[59] C. H. Lee, A. J. Gil, J. Bonet, Development of a cell centred upwind finite volume algorithm for a new conservation law formulation in structural dynamics, Computer Methods in Applied Mechanics and Engineering 118 (2012) 13-38.

[60] C. H. Lee, A. J. Gil, J. Bonet, Development of a stabilised PetrovGalerkin formulation for conservation laws in lagrangian fast solid dynamics, Computer Methods in Applied Mechanics and Engineering 268 (2014) 40-64.

[61] G. Scovazzi, B. Carnes, X. Zeng, A simple, stable and accurate tetrahedral finite element for transient, nearly incompressible, linear and nonlinear elasticity: A dynamic variational multiscale approach, International Journal for Numerical Methods in Engineering (2015). Under review. 
[62] C. H. Lee, A. J. Gil, J. Bonet, Development of a cell centred upwind finite volume algorithm for a new conservation law formulation in structural dynamics, Computers \& Structures 118 (2013) 13-38.

[63] J. Haider, C. H. Lee, A. J. Gil, J. Bonet, A first order hyperbolic framework for large strain computational solid dynamics: An upwind cell centred Total Lagrangian scheme, International Journal for Numerical Methods in Engineering (under review).

[64] T. J. R. Hughes, L. P. Franca, M. Balestra, A new finite element formulation for computational fluid dynamics: V. Circumventing the Babuška-Brezzi condition: a stable Petrov-Galerkin formulation of the Stokes problem accommodating equal-order interpolations, Computer Methods in Applied Mechanics and Engineering 59 (1986) 85-99.

[65] M. Jabareen, On the modeling of electromechanical coupling in electroactive polymers using the mixed finite element formulation, Procedia \{IUTAM\} 12 (2015) 105-115. \{IUTAM\} Symposium on Mechanics of Soft Active Materials.

[66] T. Elguedj, Y. Bazilevs, V. Calo, T. Hughes, B-bar and f-bar projection methods for nearly incompressible linear and non-linear elasticity and plasticity using higher-order nurbs elements, Computer Methods in Applied Mechanics and Engineering 197 (2008) 2732-2762.

[67] T. J. R. Hughes, The finite element method: Linear static and dynamic finite element analysis, Dover Publications, 2000.

[68] T. J. R. Hughes, Generalization of selective integration procedures to anisotropic and nonlinear media, International Journal for Numerical Methods in Engineering 15 (1980) 1413-1418.

[69] D. P. Flanagan, T. Belytschko, A uniform strain hexahedron and quadrilateral with orthogonal hourglass control, International Journal for Numerical Methods in Engineering 17 (1981) 679-706.

[70] J. Bonet, P. Bhargava, A uniform deformation gradient hexahedron element with artificial hourglass control, International Journal for $\mathrm{Nu}-$ merical Methods in Engineering 38 (1995) 2809-2828. 
[71] J. C. Simo, F. Armero, K. S. Pister, Variational and projection methods for the volume constraint in finite deformation elasto-plasticity, Computational Methods for Applied Mechanical Engineering 51 (1985) 177208.

[72] R. Stenberg, A family of mixed finite elements for elasticity problems, Numerische Mathematik 48 (1988) 513-538.

[73] J. C. Simo, F. Armero, Geometrically non-linear enhanced strain mixed methods and the method of incompatible modes, International Journal for Numerical Methods in Engineering 33 (1992) 1413-1449.

[74] J. C. Simo, F. Armero, R. Taylor, Improved versions of assumed enhanced strain tri-linear elements for 3D finite deformation problems, Computational Methods for Applied Mechanical Engineering 110 (1993) 359-386.

[75] M. A. Puso, A highly efficient enhanced assumed strain physically stabilized hexahedral element, International Journal for Numerical Methods in Engineering 49 (2000) 1029-1064.

[76] F. Auricchio, L. B. de Veiga, C. Lovadina, A. Reali, The importance of the exact satisfaction of the incompressibility constraint in nonlinear elasticity: mixed fems versus nurbs-based approximations, Computational Methods for Applied Mechanical Engineering 199 (2008) 314-323.

[77] R. L. Taylor, A mixed-enhanced formulation for tetrahedral finite elements, International Journal for Numerical Methods in Engineering 47 (2000) 205-227.

[78] C. R. Dohrmann, M. W. Heinstein, J. Jung, S. W. Key, W. R. Witkowski, Node-based uniform strain elements for three-node triangular and four-node tetrahedral meshes, International Journal for $\mathrm{Nu}-$ merical Methods in Engineering 47 (2000) 1549-1568.

[79] Q. Tiehu, Symmetrizing nonlinear elastodynamic system, Journal of Elasticity 50 (1998) 245-252.

[80] D. H. Wagner, Symmetric-hyperbolic equations of motion for a hyperelastic material, Journal of Hyperbolic Differential Equations 06 (2009) 615-630. 
[81] R. de Boer, Vektor- und Tensorrechnung für Ingenieure, Springer-Verlag, 1982.

[82] J. Bonet, A. J. Gil, R. Ortigosa, On a tensor cross product based formulation of large strain solid mechanics, International Journal of Solids and Structures 84 (2016) 49-63.

[83] R. Hill, On uniqueness and stability in the theory of finite elastic strain, Journal of the Mechanics and Physics of Solids 5 (1957) 229-241.

[84] B. D. Coleman, W. Noll, On the thermostatics of continuous media, Archive for Rational Mechanics and Analysis 4 (1959) 97-128.

[85] P. G. Ciarlet, Mathematical Elasticity. Volume 1: Three Dimensional Elasticity, 1988

[86] O. Gonzalez, A. M. Stuart, A first course in Continuum Mechanics, Cambridge University Press, 2008.

[87] P. Flory, Thermodynamic relations for high elastic materials., Transaction of the Faraday Society 57 (1961) 829-838.

[88] S. Hartmann, P. Neff, Polyconvexity of generalized polynomial-type hyperelastic strain energy functions for near-incompressibility, International Journal of Solids and Structures 40 (2003) 2767-2791.

[89] J. C. Simo, R. L. Taylor, Quasi-incompressible finite elasticity in principal stretches. continuum basis and numerical algorithms, Computer Methods in Applied Mechanics and Engineering 85 (1991) 273310.

[90] J. P. Pelteret, D. Davydov, A. McBride, P. Steinmann, Computational electro- and magneto-elasticity for quasi-incompressible media immersed in free space, International Journal for Numerical Methods in Engineering (2016). doi: 10.1002/nme.5254.

[91] A. N. Brooks, T. J. R. Hughes, Streamline upwind/Petrov-Galerkin formulations for convection dominated flows with particular emphasis on the incompressible Navier-Stokes equations, Computer Methods in Applied Mechanics and Engineering 32 (1982) 199-259.

[92] K. J. Bathe, Finite Element Procedures, Prentice Hall, 1996. 
[93] C. Sansour, On the physical assumptions underlying the volumetricisochoric split and the case of anisotropy, European Journal of Mechanics - A/Solids 27 (2008) 28-39. 